\title{
Blood-pressure management by automated drug infusion
}

Citation for published version (APA):

Hoeksel, S. A. A. P. (1998). Blood-pressure management by automated drug infusion. [Doctoral Thesis, Maastricht University]. Universiteit Maastricht. https://doi.org/10.26481/dis.19980514sh

Document status and date:

Published: 01/01/1998

DOI:

10.26481/dis.19980514sh

Document Version:

Publisher's PDF, also known as Version of record

\section{Please check the document version of this publication:}

- A submitted manuscript is the version of the article upon submission and before peer-review. There can be important differences between the submitted version and the official published version of record.

People interested in the research are advised to contact the author for the final version of the publication, or visit the DOI to the publisher's website.

- The final author version and the galley proof are versions of the publication after peer review.

- The final published version features the final layout of the paper including the volume, issue and page numbers.

Link to publication

\footnotetext{
General rights rights.

- You may freely distribute the URL identifying the publication in the public portal. please follow below link for the End User Agreement:

www.umlib.nl/taverne-license

Take down policy

If you believe that this document breaches copyright please contact us at:

repository@maastrichtuniversity.nl

providing details and we will investigate your claim.
}

Copyright and moral rights for the publications made accessible in the public portal are retained by the authors and/or other copyright owners and it is a condition of accessing publications that users recognise and abide by the legal requirements associated with these

- Users may download and print one copy of any publication from the public portal for the purpose of private study or research.

- You may not further distribute the material or use it for any profit-making activity or commercial gain

If the publication is distributed under the terms of Article $25 \mathrm{fa}$ of the Dutch Copyright Act, indicated by the "Taverne" license above, 
Blood-Pressure Management by Automated Drug Infusion 


\section{Blood-Pressure Management by Automated Drug Infusion}

\section{PROEFSCHRIFT}

ter verkrijging van de graad van doctor aan de Universiteit Maastricht, op gezag van de Rector Magnificus,

Prof. dr. A.C. Nieuwenhuijzen Kruseman, volgens het besluit van het College van Decanen, in het openbaar te verdedigen

op donderdag 14 mei 1998 om 16.00 uur

$$
\text { door }
$$

Sebastiaan Adrianus Alphonsus Petrus Hoeksel geboren te Geleen 


\section{Promotores:}

Prof. dr. ir. A. P. G. Hoeks

Prof. dr. R. S. Reneman

Co-promotores:

Dr. J. J. Schreuder

Dr. ir. J. A. Blom

Beoordelingscommissie:

Prof. dr. ir. A. Hasman (voorzitter)

Prof. dr. ir. T. G. J. Arts

Prof. dr. ir. P. P. J. van den Bosch (Technische Universiteit Eindhoven)

Prof. dr. J. G. Bovill (Academisch Ziekenhuis Leiden)

Prof. dr. P. W. de Leeuw

Financial support by the Netherlands Heart Foundation and the Stichting RESCAR for the publication of this thesis is gratefully acknowledged.

We thank Arrow International for their additional support. 


\section{Contents}

1 General introduction 9

1.1 Management of blood pressures ............ 10

1.2 This dissertation ................. 12

2 Summary of literature 15

2.1 Summary of literature . . . . . . . . . . . . . 16

2.2 Discussion ........................ 24

3 Correction for respiration artefact $\quad \mathbf{2 7}$

3.1 Introduction . . . . . . . . . . . . . . . . 29

3.2 Algorithm . . . . . . . . . . . . . . . . . . 29

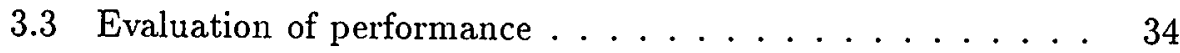

3.4 Discussion ........................ 39

4 Detection of the dicrotic notch in arterial pressure signals 41

4.1 Introduction . . . . . . . . . . . . . . 43

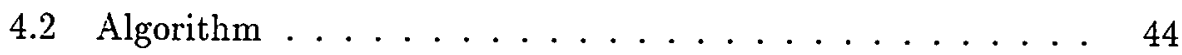

4.3 Evaluation of performance . . . . . . . . . . . . 47

4.4 Discussion ....................... 51

5 Validation of irregular blood-pressure signals $\quad 57$

5.1 Introduction . . . . . . . . . . . . . 59

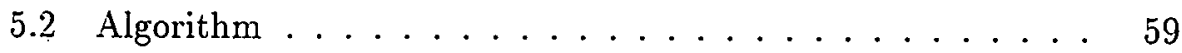

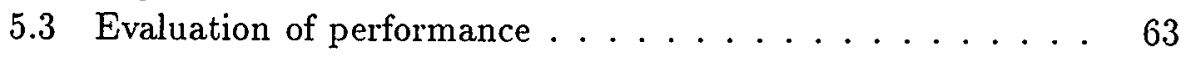

5.4 Discussion ...................... 67

6 Adaptive blood-pressure control $\quad 71$

6.1 Introduction . . . . . . . . . . . . . . . 73

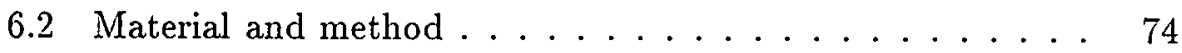

6.3 Results.................... 80 
6.4 Discussion ....................... 80

7 Control of arterial hypertension with nitroglycerin $\quad 85$

7.1 Introduction . . . . . . . . . . . . . 87

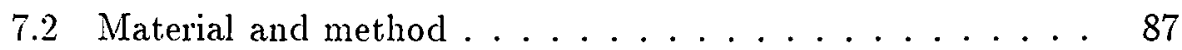

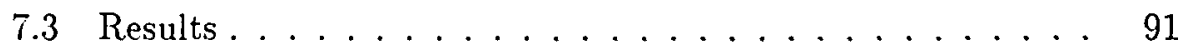

7.4 Discussion ......................... 95

8 Control of arterial hypertension with sodium nitroprusside 99

8.1 Introduction . . . . . . . . . . . . . . . . . 101

8.2 Material and method ................ 101

8.3 Results . . . . . . . . . . . . . . . . . 103

8.4 Discussion . . . . . . . . . . . . . . 105

9 Control of pulmonary artery hypertension 107

9.1 Introduction . . . . . . . . . . . . . . . . . . 109

9.2 Material and method ............... 109

9.3 Results....................... 111

9.4 Discussion ....................... 113

10 Control of arterial and pulmonary pressures 115

10.1 Introduction . . . . . . . . . . . . . . . 117

10.2 Material and method ................. 118

10.3 Results . . . . . . . . . . . . . . . . 125

10.4 Discussion . . . . . . . . . . . . . . 130

11 Computer control versus manual control 135

11.1 Introduction . . . . . . . . . . . . . . . . . . . . . . . . . . 137

11.2 Material and method . . . . . . . . . . . . 138

11.3 Results . . . . . . . . . . . . . . . . . . . . 140

11.4 Discussion . . . . . . . . . . . . . . . 141

12 General discussion $\quad 145$

$\begin{array}{ll}\text { Appendix } & 149\end{array}$

Bibliography 153

$\begin{array}{ll}\text { Summary } & 163\end{array}$ 
Chapter 1

General introduction 


\subsection{Management of blood pressures}

During and shortly after cardiac surgery information about cardiac pump function is obtained by monitoring systemic arterial and pulmonary artery blood pressures continuously and cardiac output intermittently. The cardiac output needs to be sufficient to ensure adequate perfusion of all organs and the systemic arterial blood pressure should be high enough to ensure adequate perfusion of the coronary arteries. Too high a systemic arterial blood pressure, however, should be avoided to limit perioperative blood loss and the load on the left ventricle and hence its oxygen consumption. In the absence of pulmonary disease, mean pulmonary artery blood pressure can be used as an indicator of left ventricular preload. Too low a mean pulmonary artery blood pressure indicates too low a preload, whereas sudden increases in pulmonary artery blood pressure are indicative of acute failing of left ventricular pump function. One of the anaesthesiologist's tasks is continuously to monitor the haemodynamic variables and to infuse vasoactive and contractility enhancing drugs when variables fall outside the optimum range for a patient.

For the management of blood pressure, closed-loop control by computer has been proposed to relieve the anaesthesiologist of the time-consuming task of continuously adjusting drug infusion rates. Closed-loop control involves automatic assessment of mean blood pressure and calculation of drug infusion rates needed to control blood pressure around a desired target level. Drugs are administered to the patient by a computer-controlled infusion pump. A simplified model, usually of a first order, is used to predict the patient's response to changes in the drug infusion rate. An adaptive control strategy is used to identify the parameter values of the patient model online and to optimize adjustment of the closed-loop system for the specific patient. Blood pressures are routinely measured invasively by means of external pressure transducers and a fluid-filled line connected to a radial artery cannula or a pulmonary artery catheter. Signal validation algorithms must be used to ensure that the closed-loop system does not respond to invalid pressure measurements, for example those caused by flushing of the fluid-filled line or electrical interference from the coagulation equipment.

Closed-loop control may provide more stable blood-pressure control than manual control because a computer, unlike man, can be optimized for a single repetitive task, does not lose concentration after a prolonged period and is not distracted. Furthermore, closed-loop control allows the clinician to concentrate on other more complex patient-related tasks. For 
the control of postoperative hypertension in cardiac surgical patients, for example, closed-loop control reduces nursing time spent adjusting vasodilator infusion rates (Mitchell 1982; Cosgrove III et al. 1989), decreases the incidence of hypotension caused by therapy, reduces postoperative bleeding, decreases transfusion requirements and probably lowers the incidence of re-exploration for postoperative bleeding (Chitwood et al. 1992).

From review articles (Katona 1982; Linkens and Hacisalihzade 1990; O'Hara et al. 1992; Jastremski et al. 1995) and recently published papers on the subject of closed-loop blood-pressure control it can be concluded that in general control designs have been evaluated in computer models or in animal studies and only a limited number of designs have been tested clinically. Introducing closed-loop blood-pressure controllers in a clinical setting may pose problems owing to noise and changes in blood pressure unrelated to changes in drug infusion rate. Those control designs that have been tested clinically mainly concerned postoperative systemic hypertension in cardiac surgical patients (de Asla et al. 1985; Reid and Kenny 1987; Cosgrove III et al. 1989; Bednarski et al. 1990; Chitwood et al. 1992) and often in a relatively small number of patients. Control of blood pressure during cardiac surgery is more complicated, because here the patient's condition may change more rapidly owing to the many interventions occurring during the surgical procedure, requiring a more aggressive control of blood pressure than in the intensive care unit. Fewer attempts have been made to control systemic arterial blood pressure during cardiac surgery (Blom 1991; Martin et al. 1992b; Meijers et al. 1997) and only the recent study by Meijers et al. (1997) claimed an acceptable control performance in all patients. Surprisingly, the problem of invalid blood-pressure measurements is not addressed by most researchers. Jastremski et al. (1995) therefore concluded correctly that closed-loop control of blood pressure is still in an innovative phase and that in particular control algorithms and sensor validation require further improvement.

Though most control designs are for the control of systemic hypertension, the ultimate goal of a closed-loop controller ought to be the control of overall cardiac pump function, as indicated by systemic arterial and pulmonary artery blood pressure and cardiac output. Closed-loop control of cardiac output is not yet possible, because the techniques at present available for assessing cardiac output do not allow reliable continuous measurement. Control of cardiac preload has been reported by Sheppard et al. (1968) who controlled left and right atrial pressures by automating the infusion of blood volume in postoperative cardiac surgical patients. No closed-loop system has been described for the control of pulmonary artery 
blood pressure as an indicator of left ventricular preload. Only a few theoretical designs have been proposed for the simultaneous control of more than one haemodynamic variable. Voss et al. (1987) and Yu et al. (1992) both described closed-loop systems that control systemic arterial pressure and cardiac output by simultaneously varying the infusion rates of a vasodilator and an inotropic drug. Both systems were evaluated in dogs using an aortic flow probe to continuously measure cardiac output.

\subsection{This dissertation}

The aim of the research described in this dissertation was to develop and evaluate clinically a closed-loop system that simultaneously adjusts the infusion rates of vasoactive and contractility enhancing drugs in such a way that systemic arterial and pulmonary artery blood pressures are maintained close to preset target levels. Chapter 2 gives a detailed summary of the current situation concerning progress in closed-loop blood-pressure controller technology. This dissertation can then be divided into two parts. The first part is concerned with the assessment and the automatic interpretation and validation of invasively measured radial and pulmonary artery bloodpressure signals. The second part describes the development and clinical evaluation of several closed-loop blood-pressure controllers.

Chapter 3 describes a method for validating pulmonary artery pressure signals in the presence of cyclic disturbances induced by mechanical ventilation. Chapter 4 presents a novel algorithm for the detection of the duration of left ventricular ejection from radial artery pressure signals. Current validation techniques do not work properly in patients with irregular heart rates. In chapter 5 we therefore investigate the feasibility of timing ventricular ejection in order to validate blood-pressure signals when considering irregular heart rates.

Closed-loop blood-pressure controllers are adaptive and implicitly assume that patient-model parameters can be identified during a control session. In chapter 6 we have evaluated the validity of this assumption for blood-pressure data obtained during cardiac surgery. In chapter 7 we describe the clinical evaluation of a closed-loop system for the control of systemic hypertension during cardiac surgery using the vasodilator nitroglycerin. This single-drug control strategy was extended for the control of systemic hypertension during cardiac surgery by means of sodium nitroprusside (chapter 8) and for the control of pulmonary hypertension during cardiac surgery by means of nitroglycerin (chapter 9 ). Chapter 10 describes 
the integration of several single-drug closed-loop systems in a multipledrug infusion system that simultaneously controls systemic arterial and pulmonary artery pressures. The effects of closed-loop control of systemic hypertension during cardiac surgery are investigated in chapter 11 . The dissertation ends with a general discussion (chapter 12). 
Chapter 2

Summary of literature 


\subsection{Summary of literature}

A number of attempts have been made in the past decades to automate routine tasks in a clinical environment (Katona 1982; Linkens and Hacisalihzade 1990; O'Hara et al. 1992). Computer systems have been used to control heart-rate trajectory during stress testing (Marwick 1995), fluid adjustment during haemodiafiltration (Parkin et al. 1994), heart rate with a closed-loop cardiac pacemaker (Pichlmaier et al. 1992), the infusion of muscle relaxants (Stinson et al. 1994), triggering of intraaortic balloon pumping (Zelano et al. 1990) and perfusion pumps during cardiopulmonary bypass (Beppu et al. 1995).

An important development has been the introduction of computer controllers for the control of systemic arterial blood pressure (Katona 1982; Linkens and Hacisalihzade 1990; O'Hara et al. 1992; Jastremski et al. 1995). A blood-pressure controller (figure 2.1 ) basically comprises a bloodpressure sensor, a control algorithm and an infusion pump. The first part of this synopsis focuses on algorithms for evaluating the quality of invasively measured blood-pressure signals. The second part describes our knowledge of the patient's response to vasoactive drugs. The third part describes the various blood-pressure control strategies.

\subsubsection{Rejection of measurement artefacts}

In closed-loop blood-pressure control the validity of the pressure measurements must be guaranteed in order to prevent the controller from changing its control signal in response to distorted measurements. Pressure measurements might for example be distorted by a disconnected or malfunctioning sensor, movement of the patient or electrical interference from coagulation equipment. A drift in the pressure sensor, causing an offset between the measured and the true pressure, cannot be detected automatically and should be identified by the clinician.

Signal-validation algorithms often use the cyclic nature of physiological signals to reject measurement artefacts. First, each period of the raw signal is described by a set of characteristic features. The mean and fluctuations of these derived features over time provide evidence for the validity of the measured signal and are used to reject those segments of the signals with deviating characteristics. Arrhythmias in electrocardiogram recordings, for example, are detected when the actual period time differs substantially from the predicted period time (Woolfson 1991). For blood-pressure signals physiological markers, such as systolic and diastolic pressures, can be used 


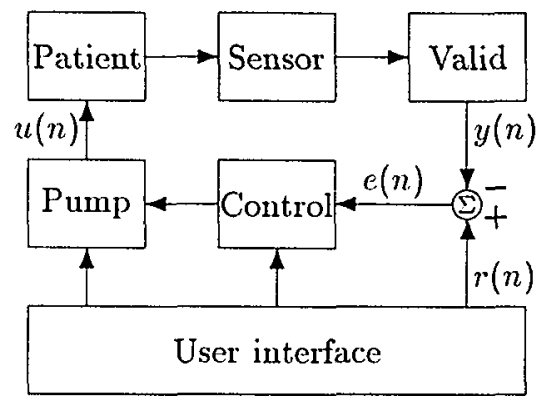

Figure 2.1: Setup of a blood-pressure controller. The sensor measures the patient's pressure signal. A validation routine (Valid) tests the validity of the pressure signal and calculates the mean pressure value, $y(n)$. A control law calculates the infusion rate, $u(n)$, based on the observed deviations, $e(n)$, between $y(n)$ and the target pressure, $r(n)$. The control systems sends $u(n)$ to a computer-controlled infusion pump. The user interface allows the user manually to set an infusion rate, to activate the controller and to change $r(n)$. In addition the user interface displays the controller's status and a trend display of all relevant signals.

to characterise each pressure beat. Arterial pressure beats at a regular heart rate can be validated by rejecting the pressure beats, the features of which deviate substantially from the averaged feature values of previous pressure beats (Blom 1990).

Systems based on artificial intelligence to reject invalid measurements have also been proposed. Neural networks can be taught to recognize invalid blood-pressure signals (Pike and Mustard 1992; Odaka et al. 1994; Prentza and Wesseling 1995). Apart from large computational efforts, a neural network approach requires a realistic training set and a relatively long learning time for each patient.

\subsubsection{Modelling patient response}

An adequate mathematical model is needed to predict the blood-pressure response to changes in the drug infusion rate before a blood-pressure control system can be developed. Using correlation analysis and pseudo-random binary changes in infusion rate, Sheppard (1980) found that the bloodpressure response to the vasodilator sodium nitroprusside (SNP) can be modelled by a first-order transfer function

$$
H(s)=\frac{Y(s)}{U(s)}=\left(1+\alpha \mathrm{e}^{-T_{c} s}\right) \mathrm{e}^{-T_{d} s} \frac{K}{1+\tau s}
$$


where $Y(s)$ and $U(s)$ are the Laplace transforms of the induced pressure change, $y(n)$, and the drug infusion rate, $u(n)$, respectively. $T_{d}$ represents the initial transport lag from the injector site to the effect compartment and is approximately equal to one circulation time. $K^{*}$ is the patient's sensitivity and relates the drug concentration in the effect compartment to the decrease in blood pressure. The drug elimination from the effect compartment is described by a first-order process with time constant $\tau$. The recirculation factor $\alpha$ is the ratio of drug quantity that recirculates within $T_{c}$ seconds. In practice the recirculation factor $\alpha$ is often neglected when model 2.1 is used for the design of a closed-loop system. (Stern et al. 1985; Prys-Roberts and Millard 1990; Blom 1991; Meijers et al. 1997). Beside variations in time constant, $\tau$, and time till onset of drug response, $T_{d}$, it is mainly the patient's sensitivity that varies strongly between and within patients (Sheppard 1980; Prys-Roberts and Millard 1990; Blom 1991). For example, for SNP the patient's sensitivity, $K$, may vary by a factor 80 (Blom 1990). This wide range in $h^{-}$might partly be attributed to the dependency of the drug response curve to the patient's age (Wood et al. 1987), pathology and circulatory state (Friederich and Butterworth 1995). Owing to the wide range and variability of $K$, blood-pressure controllers should use an adaptive control strategy (section 2.1.3).

Other drugs that were used in a closed-loop system whose response could satisfactorily be described by a first-order model include nitroglycerin (NTG) (Colvin and Kenny 1989), halothane (Smith and Schwede 1972), isoflurane (Prys-Roberts and Millard 1990) and phenylephrine (Millard et al. 1987). The response of some drugs cannot be described by a firstorder model, necessitating an extended model description. Dopamine, for example, decreases arterial pressure at low infusion rates $\left(<3 \mu \mathrm{g} \mathrm{kg}^{-1}\right.$ $\min ^{-1}$ ), whereas arterial pressure increases at higher infusion rates (Gingrich and Roy 1991).

A first-order model may be regarded as a linearization around a working point and a different response within one patient might therefore be expected as pressure levels change. The sensitivity to SNP, for example, appears to decrease at lower pressure levels (Blom 1990). Additional nonlinear behaviour may be introduced by the interactions with the patient's internal pressure regulation mechanisms (Guyton et al. 1972; Miller et al. 1977). Todd et al. (1982) illustrated some of these interactions in dogs by inducing hypotension with either SNP or NTG. After thirty minutes infusion was abruptly stopped and with SNP arterial pressure quickly returned to control values, but with NTG rebound pulmonary hypertension was observed and more than two hours were required before baseline arte- 


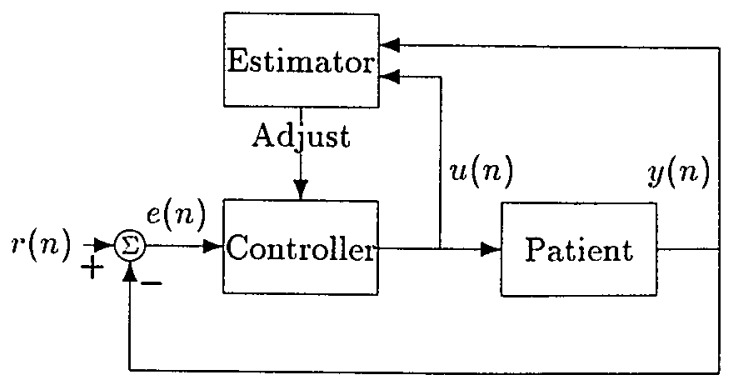

Figure 2.2: An adaptive control scheme. The parameters of the patient model are estimated online. The estimated parameters are used to adjust the controller's behaviour. $y(n)$, measured blood pressure. $r(n)$, target pressure. $e(n)$, error between pressure and target. $u(n)$, drug infusion rate.

rial pressure was reached.

\subsubsection{Control of blood pressure}

For the control of blood pressure a fixed gain control design, i.e. a design with time-invariant design parameters, does not provide accurate control in all patients owing to the large variability in response to vasoactive drugs between patients and within patients over time (section 2.1.2). For this reason most blood-pressure controllers use an adaptive control scheme (figure 2.2) (Isaka and Sebald 1993). With adaptive control, patient model parameters are estimated recursively for each new pressure sample and the controller is adapted accordingly ( $\AA$ ström and Wittenmark 1995 ).

\section{Proportional-integral-derivative control}

In proportional-integral-derivative (PID) control, the instantaneous infusion rate depends on the error, $e(t)$, between the measured pressure and the target pressure and on the integral and the derivative of that error. The mathematical formulation of the PID control law in the continuous time domain is given by

$$
u(t)=G\left(e(t)+\frac{1}{G_{i}} \int_{0}^{t} e(\tau) d \tau+G_{d} \frac{d e(t)}{d t}\right)
$$


The PID parameters $G, G_{i}$ and $G_{d}$ are chosen such that control is suffciently fast without causing unstable or oscillatory behaviour ( $\AA$ ström and Hagglund 1995). For the control of blood pressure, the derivative gain, $G_{d}$, is often set to zero because the noisy time derivative of $e(t)$ does not provide valuable information. Sheppard (1980) used a proportional-integral (PI) control law combined with gain scheduling to control the infusion of SNP in hypertensive postoperative cardiac surgical patients. A decision table was added to bias the PI controller toward minimizing infusion rates. The rules in the decision table modified both the control gain and the rate of change in infusion rate, dependent on the current value of $e(t)$. Various controllers have been based on this control strategy (de Asla et al. 1985; Reid and Kenny 1987; Chaudhrim et al. 1992). Colvin and Kenny (1989) developed a PI controller that allowed the user to select between the two vasodilators SNP and NTG. Rosenfeldt et al. (1986) developed a PI bloodpressure controller that incorporated the option of control-performance adjustment by manually alteration of algorithm design parameters. Blom (1990) described an adaptive mechanism that analysed the rate of pressure decay toward the target pressure. PI control gain, inversely related to the estimated patient's sensitivity, was adaptively updated when the pressure decay was either too fast or too slow.

\section{Optimum control}

An optimum controller changes its control signal, $u(n)$, to optimize a performance index ( $\AA$ ström and Wittenmark 1995). In minimum variance control, the control signal $u(n)$ is chosen such that the performance index

$$
J=\mathrm{E}\left[(\hat{y}(n \mid n-1)-r(n))^{2}\right]
$$

is minimized, where $E[\cdots]$ denotes the expectation operator, $r(n)$ is the desired blood pressure and $\hat{y}(n \mid n-1)$ is the one-step-ahead prediction of the patient's blood pressure, calculated from previous input and output data and a model describing the patient's response (for example model 2.1). An optimum controller is made adaptive by recursively estimating the patient model parameter values (Ljung and Söderström 1983; Aström and Wittenmark 1995). Direct application of the optimum control signal, minimizing $J$ of equation 2.3, might place a too strong confidence in the quality of the patient model and therefore a smoothing filter is often used (Prys-Roberts and Millard 1990). The performance index 2.3 can also be extended to include for example a penalty for the total amount of drug given (Stern 
et al. 1985) or for the fluctuations in the control signal $u(n)$ (McInnis and Deng 1985; Arnsparger et al. 1983). Long-range adaptive control has been proposed to minimize the trajectory of future pressure responses (Kwok et al. 1995).

Arnsparger et al. (1983) used a recursive least-squares estimator combined with a self-tuning minimum variance control law to control systemic arterial pressure in dogs. Prys-Roberts and Millard (1990) used a selftuning optimum controller to control arterial hypertension in 19 patients scheduled for either plastic surgery or neurosurgical procedures. Optimum control has also been proposed to control multiple clinical variables. McInnis and Deng (1985) described a self-tuning optimum control strategy for the simultaneous control of arterial and venous pressures using a vasodilator and an inotropic drug. Their system was tested on a computer model. Voss et al. (1987) described a self-tuning controller for the control of arterial pressure and cardiac output using dopamine and SNP. Their strategy was tested in dogs, measuring cardiac output with an aortic flow probe. The authors concluded that the control strategy was feasible, but that further study was needed before clinical applications should be attempted.

\section{Multiple-model adaptive control}

Multiple-model adaptive control can be used to limit the online estimated patient model to a finite set of models. Each model is associated with an optimally tuned regulator (figure 2.3). The final infusion rate is a weighted function of all regulator outputs. The weight given to each regulator is estimated by a recursive identification routine and expresses the likelihood that the patient's response can be described by the associated model.

He et al. (1986) described a multiple-model adaptive controller for the control of SNP infusion rates and evaluated the system using computer simulations and dogs. Martin et al. (1987) described a multiple-model adaptive control strategy for the control of blood pressure using SNP. Later a modified version of this multiple-model adaptive controller was employed to control arterial pressure during cardiac surgery (Martin et al. 1992b). Yu et al. (1992) described a multiple-model adaptive controller for the simultaneous control of arterial pressure and cardiac output. Animal studies indicated that transitions between the various models were robust enough. Cardiac output was measured with either an aortic flow probe or by means of transoesophegal echo. 


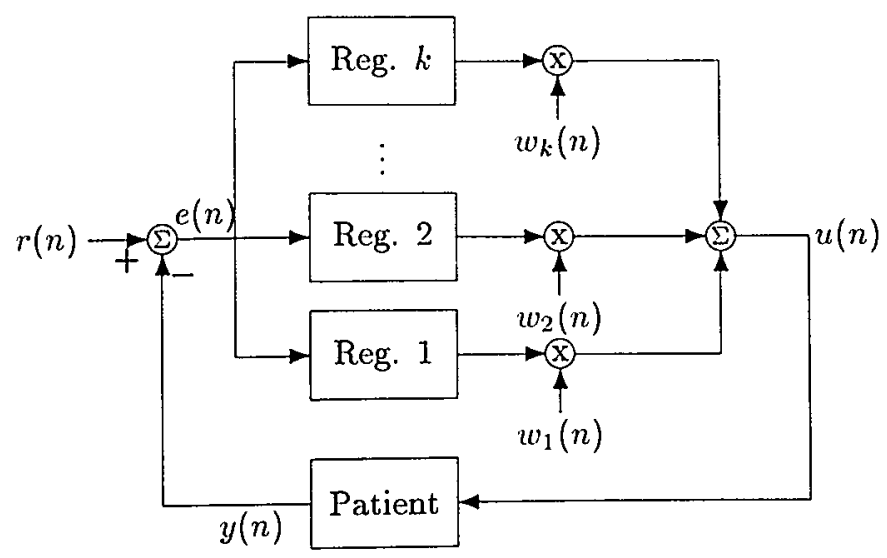

Figure 2.3: Control scheme of a multiple-model adaptive controller. The weight $w_{k}(n)$ gives the likelihood that the $k^{l h}$ model, that was used to tune the $k^{t h}$ regulator (Reg. ki), describes the true patient model. The final infusion rate $u(n)$ is the weighted sum of all regulator outputs. If for example the weight $w_{1}$, associated with 'very sensitive', is largest then regulator 1 is most active and will provide a cautious control.

\section{Estimating delay time}

The value of the time delay, $T_{d}$, between the start of drug infusion and the onset of blood-pressure response cannot easily be estimated online and is therefore often assumed to be a known constant. Stern et al. (1985) evaluated a blood-pressure controller that estimated the delay time in dogs online, by finding which of a range of possible delay times best fits the true patients' response. Martin et al. (1987) described a method to estimate the delay time from the pressure response to a stepwise change in target pressure.

\section{Rule-based control}

Many blood-pressure control designs do not function satisfactorily in all patients, although the anaesthesiologist is capable of controlling blood pressure in practically all patients. This apparent contradiction has led to the development of controllers based on artificial intelligence techniques which try to mimic the anaesthesiologist's decision-making ability by incorporating intelligent reasoning. A rule-based controller tries to capture the anaesthesiologist's reasoning in a set of empirically derived if-then rules. 
Hammond et al. (1979) designed a blood-pressure control system based on a set of rules that analysed the distance from the target pressure to determine the change in drug infusion rate. The system contained rules like:

if 'pressure close to target' then 'keep infusion constant'

if 'pressure above target' then 'increase infusion rate by $5 \%$ '

Packer et al. (1987) described a similar system, but used a look-up table to automate the existing protocol for the manual adminstration of SNP.

\section{Fuzzy-logic control}

Fuzzy-logic is another attempt to emulate the clinician's decision-making ability in a computer program. In a fuzzy-logic controller the inference process is preceded by a fuzzification process which translates mathematical statements into fuzzy variables. Unlike with two-valued boolean logic, a variable in fuzzy-logic can be member of multiple classes. A weight describes the degree of membership to each class. The inference part of the fuzzy-logic controller reasons with the fuzzified statements and derives a fuzzy output. To reason with fuzzified statements a special logic has been developed by Zadeh (1978). A defuzzification process translates the fuzzified output into mathematical changes in the infusion rate.

In a small group of patients undergoing abdominal surgery Zbinden et al. (1995) used fuzzy logic to manage systemic arterial pressure by automatically adjusting the inhaled dose of the gas isoflurane. Ruiz et al. (1993) used fuzzy logic to automate the infusion of SNP for the control of postoperative hypertension in 40 cardiac surgical patients. The theoretical design of a multi-variable fuzzy-logic controller was discussed by Held and Roy (1995). Ying et al. (1992) discussed the design of a blood-pressure controller that combines fuzzy and rule-based logic and showed that the system can be translated into a nonlinear PI controller thereby greatly reducing computation time.

\section{Neural network control}

There is an increasing interest in neural networks to model and control nonlinear systems (Hunt et al. 1992). A neural network consists of layers of neurons that are connected by several weighted links. A neural network can be trained by determining the values of all weights using a given clataset. Neural networks have only recently been proposed for the control of blood pressure by Chen et al. (1997) who described a control strategy that combined two neural networks layers using rule-based logic. 


\section{Supervisory control}

A blood-pressure control strategy might provide adequate control under normal circumstances, but in clinical practice rapid changes in the patient's haemodynamic condition might be observed due to surgical manipulations or changes in the patient's condition. Most researchers have therefore recognized the importance of an independent supervisory safety shell that defines action when unmodelled changes in haemodynamics occur. Martin et al. (1992a) described a supervisory safety shell that limits changes in drug infusion rates and detects rapid changes in blood pressure. Blom (1990) described SIMPLEXYS, a real-time expert system development tool that facilitates the incorporation of expert knowledge into real-time applications. Control systems designed with the aid of SIMPLEXYS have been used to control arterial hypertension during cardiac surgery (Blom 1991; Meijers et al. 1997).

\subsection{Discussion}

It can be concluded that many controllers have been evaluated in model studies or animal studies, but that only a limited number of controllers has reached the level of clinical testing. Systems that were tested in a clinical setting mostly controlled systemic arterial hypertension in postoperative cardiac surgical patients. The vasodilator drug SNP was most frequently used, although the use of NTG has also been reported (Colvin and Kenny 1989). Closed-loop blood-pressure control during cardiac surgery is more challenging, because here the patient's condition may change rapidly because of the many interventions occurring during the surgical procedure, requiring a more aggressive control of blood pressure than in the intensive care unit. Preliminary investigations into the feasibility of computer control of SNP infusion rates during cardiac surgery by Blom (1991) and Martin et al. (1992b) showed that control of mean arterial pressure (MAP) was adequate, though both controllers were manually overruled by the anaesthesiologist at several occasions. Meijers et al. (1997) recently described an improvement of the system originally described by Blom (1991) and claimed an acceptable control performance in all patients. Sheppard et al. (1968) evaluated a closed-loop system for the control of cardiac preload, but until now no attempts have been made to closed-loop control pulmonary artery blood pressure. Designs for simultaneous control of more than one clinical variable have been described independently by Voss et al. (1987), Yu et al. (1992) and Held and Roy (1995), but no evaluations in a clinical 
setting were reported.

Control of blood pressure requires an adaptive control strategy to adjust the controller's design parameters during a control session. The performance of an adaptive controller depends on the assumption that model parameters can adequately be identified online and within a limited time span. In a clinical setting parameter estimates based on short-time segments might become unreliable as a result of unmodelled changes in pressure, induced by surgical manipulations, changes in patient condition and infusion of other drugs. Therefore care is needed in the interpretation of results from online estimations. It is not unlikely that an increase in blood pressure caused by a painful stimulus will make an adaptive controller learn that a vasodilator increases blood pressure, leading to an unstable control design. Multiple-model adaptive control tries to prevent the problem of poor iclentifiability by restraining parameter estimations to physiological values, but this does not solve the underlying problem of an unidentifiable patient model.

Controllers based on artificial intelligence (rule-based, fuzzy-logic and neural networks) have been proposed to circumvent the problems with adaptive control indicated above. The descriptive formulation of the control problem is comprehensible for the clinicians because the system speaks their language. For technicians, systems based on artificial intelligence require a new way of thinking, which makes debugging controllers based on artifcial intelligence reasoning tricky. Limitations in computation time form an additional problem in control systems based on artificial intelligence techniques. Artificial intelligence techniques are often combined with existing control strategies. In this way rule-based logic can be used to define a supervisory safety shell that determines the controller's response to unexpected episodes of severe hypotension or hypertension (Blom 1990; Martin et al. 1992a; Held and Roy 1995).

The evaluation of the quality and the validity of the measured bloodpressure signals is a problem that has not received much attention by most researchers. Yet, it forms an essential aspect of a blood-pressure controller in a clinical setting because the feedback loop relates the administered drug infusion rate directly to the measured pressure. Blom (1990) described a method for the validation of arterial pressure beats, but this technique fails for the pulmonary artery pressure owing to strong modulation of the pulmonary artery blood-pressure signal with ventilation. In addition, the interpretation of pressure signals from patients with an irregular heartrate has not yet been addressed. Closed-loop control of blood pressure in patients with an irregular heart-rate is important as this group of pa- 
tients with unstable haemodynamics benefits most from computer control of haemodynamics.

The single-drug blood-pressure controllers that are described in this dissertation are based on an SNP-based blood-pressure controller originally described by Blom (1990). The original system used a PI regulator and rule-based logic to adapt or overrule the controller when necessary (Blom 1990; Blom 1991). The rule base overruled the PI regulator during sudden large increases and decreases in MAP. Moreover, during infusion pump problems (e.g. syringe empty or infusion line occluded) and invalid pressure measurements the rule base first alerted the clinician and later switched control back to manual mode. The time-out alarms of the infusion pumps provided an independent software watchdog in the unlikely situation that the computer system went down. In a preliminary evaluation with 30 patients undergoing cardiac surgery (Blom 1991) the clinicians considered the system slow and overcautious, making it more ideal for the intensive care environment. The implemented expert knowledge proved to be correct, though the adaptation of the PI control gain was overly cautious in some cases, prompting the clinician temporarily to take over control on average once per case. The signal validation routines correctly interpreted the radial artery pressure pulse, but the validation algorithm could not handle pressure beats at an irregular heart-rate.

To achieve the purpose of this study, several additions are needed to optimize the original SNP-based blood-pressure controller system. Signal validation routines have to be extended to handle pressure signals at an irregular rate and pulmonary pressure signals (chapters 3, 4 and 5). The speed of control has to be increased to guarantee an acceptable speed of control during cardiac surgery. The original system controlled systemic arterial pressure using only SNP and has to be extended to automate other drugs, like the infusion of NTG for the control of systemic arterial and pulmonary artery blood pressures (chapter 7, 8 and 9). Eventually these single-drug controllers can be combined in one multiple-drug infusion system (chapter 10).

Blood-pressure controllers need built-in expert knowledge to define a supervisory safety shell. Expert knowledge was originally implemented using the real-time expert system development tool SIMPLEXYS (Blom 1990). SIMPLEXYS is a general-purpose expert system development tool and could be simplified even more for our control application. For our application we therefore have to design an environment that allows for more easy implementation of expert knowledge (see appendix). 


\section{Chapter 3}

\section{Correction for respiration artefact in pulmonary blood-pressure signals of ventilated patients}

S. A. A. P. Hoeksel

J. A. Blom

J. R. C. Jansen

J. J. Schreuder 


\section{Abstract}

Objective. To develop an algorithm that corrects pulmonary artery pressure signals of ventilated patients for the respiration artefact. The algorithm should test the validity of the pulmonary pressure signal and differentiate between the cyclic respiration artefact and true measurement artefacts. Material and method. The shape of each pulmonary pressure beat is described by eight characteristic features, including mean pressure value and the systolic and diastolic timing and pressure values. The features are corrected for the respiration artefact by fitting them in a least squares sense on the first and second harmonics of the ventilator frequency. The corrected features are used by a signal validation algorithm, which adds a validity flag to each pressure beat. The validation algorithm rejects pressure beats with sudden changes in their shape but adapts itself when the changes persist. Results. The performance of the correction and validation technique was evaluated using pulmonary artery pressure signals of 30 patients who were scheduled for open-heart surgery. The algorithm correctly recognized as invalid data those pressure signals disturbed by coagulation, surgical manipulations or flushes of the pressure line. The algorithm marked on average $77 \pm 11 \%$ of the pulmonary pressure beats as valid.

Conclusions. The validation algorithm marked sufficient pressure beats as valid to update a trend display every five seconds. The correction algorithm enabled the validation algorithm to differentiate between true measurement artefacts and the respiration artefact.

Keywords algorithm • signal processing • clata recording • mechanical ventilation - pulmonary pressure 


\subsection{Introduction}

The reliability of information provided by monitoring equipment is vital for the treatment of the critically ill. The aspect of signal reliability has gained even more importance with the introduction of closed-loop systems in a clinical environment (O'Hara et al. 1992; Linkens and Hacisalihzade 1990). Artefact rejection algorithms ensure that closed-loop systems do not respond to any non-physiological change in the measured input signal.

Arterial blood pressure is the most frequently used feedback signal (Colvin and Kenny 1989; Martin et al. 1992b; Chitwood et al. 1992) in a clinical setting. Blood-pressure signals can be distorted by air bubbles or blood clotting in the pressure line, electromagnetic interference from the coagulation equipment, surgical interventions, movement of the patient, blood sampling and flushing of the pressure line. Under physiological circumstances the shape of an arterial pressure beat does not change drastically, thus detection of artefacts is possible using pattern recognition to detect and reject those pressure beats that differ sufficiently from previous beats.

The validation of pulmonary artery pressure signals is more complicated than the validation of systemic arterial pressure signals because the former are changed by the respiration artefact. We developed a filtering technique that corrects characteristic features of pulmonary pressure beats for the respiration artefact during mechanical ventilation. The corrected features are used to test the validity of each pulmonary pressure beat on a beatto-beat basis. As a possible application of the algorithm we designed a five-second trend display of the validated average pulmonary pressure.

\subsection{Algorithm}

The algorithm can be separated into three parts: the first part of the algorithm detects each beat in the pulmonary artery pressure signal and extracts characteristics features; the second part corrects these characteristic features for the respiration artefact and the last part validates each pulmonary pressure beat using the corrected features.

\subsubsection{Extraction of features from the pulmonary pressure signal}

The first part of the algorithm detects pressure beats in the pulmonary pressure signal. The digitized pressure signal is passed through a lowpass 


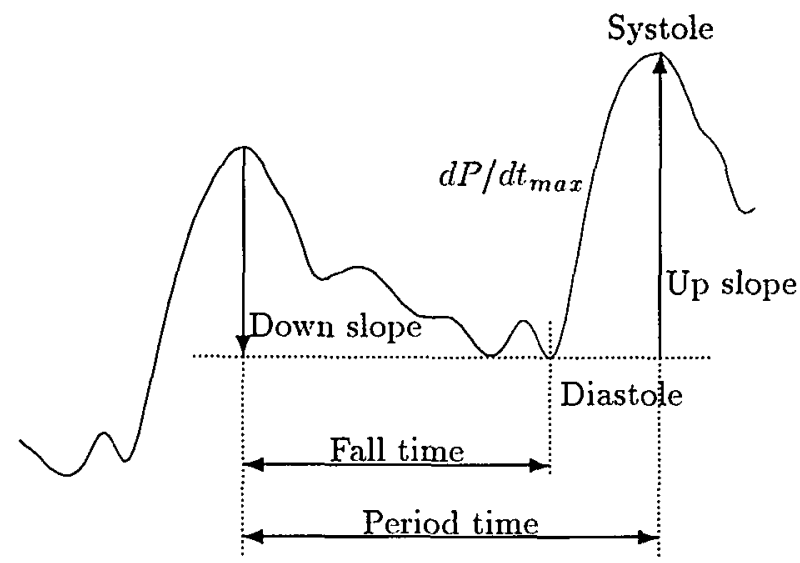

Figure 3.1: The shape of a pulmonary pressure beat is described by eight characteristic features. MPAP is the average pressure between two successive systoles.

third-order Butterworth filter with its cutoff frequency at 10 Hertz. This filter provides a smooth pressure signal and does not significantly suppress systolic pressure values. Local minima and maxima in the pressure signal are detected when its first-order time-derivative crosses zero. At each maximum an upstroke pressure is calculated as the difference between the current maximum and the previous minimum. A new systole is detected if the upstroke pressure is larger than $80 \%$ of the moving average of previous systolic upstroke pressures.

We defined eight characteristic features to describe the shape of a pulmonary pressure beat (figure 3.1). A beat is defined between two systoles since each new beat is detected at its systole. The more commonly used time period between two consecutive diastoles can, however, be derived from the timing features of two successive periods. Mean pulmonary artery pressure (MPAP) is the average pressure value between consecutive systoles. Systolic and diastolic pressures follow from the maximum and minimum of the systolic upstroke. Up-slope and down-slope pressures are derived from the diastolic pressure of the current period and the systolic pressures of the previous and the current periods. The period time is the time between two consecutive systoles and the fall time is the time difference between the start of the period and the diastole of that period. $d P / d t_{\max }$ is found by continuously updating it during the systolic upstroke with each $d P / d t$ larger than the current $d P / d t_{\max }$. 


\subsubsection{Filtering of pressure beats}

The features derived from pulmonary pressure beats show a strong fluctuation with the ventilator signal. Mechanical ventilation causes fluctuations in intrathoracic pressures, which influence the venous return, the filling of the atria and the impedance of the pulmonary vascular bed (Foëx 1980). As a consequence, both offset and pulse pressure of the pulmonary artery pressure signal fluctuate during ventilation. The features should be corrected for this fluctuation to enable distinction between the respiration artefact and true measurement artefacts. Averaging of the features will not work since this will also filter out measurement artefacts. The periodic property of the respiration artefact in ventilated patients can be used to describe a feature $y$ by a DC term plus a sine with $m$ higher harmonics and an unknown phase shift,

$$
y\left(t_{n}\right)=\frac{a_{0}}{2}+\sum_{k=1}^{m}\left(a_{k} \cos \left(\omega k t_{n}\right)+b_{k} \sin \left(\omega k t_{n}\right)\right)+e\left(t_{n}\right)
$$

where $\omega=2 \pi f$ and $e\left(t_{n}\right)$ is a random noise process. The ventilator frequency $f$ can either be read from the ventilator or extracted from the fluctuations in the pulmonary pressure signal. The variables $a_{0}, a_{k}$ and $b_{k}$ are allowed to vary slowly in time to reflect changes in the patient's condition.

Rather then estimating all parameters simultaneously, $a_{0} / 2$ is determined using a lowpass filter and then $a_{k}$ and $b_{k}$ are determined using a least squares fitting procedure. The lowpass filter is implemented as the integral over one ventilator period divided by the period time of one ventilator cycle,

$$
\frac{a_{o}}{2}=\frac{1}{T} \int_{l_{n}-T}^{l_{n}} y(\tau) d \tau
$$

where $T=1 / f$. Integral (3.2) is solved numerically using linear interpolations. The ventilator frequency is calculated counting the number of positive zero-crossings of the lowpass filtered MPAP feature with the MPAP feature itself (Papoulis 1991).

Preliminary results indicated that for the estimation of $a_{k}$ and $b_{k}$ in (3.1) the first two harmonics (i.e. $m=2$ ) would suffice. Given $a_{0} / 2$ from (3.2), the parameters $a_{k}$ and $b_{k}$ can be found by minimizing the cost function

$$
J\left(\theta, t_{n}\right)=\sum_{k=1}^{n} \lambda^{n-k}\left[y\left(t_{k}\right)-\frac{a_{0}}{2}-\theta\left(t_{k}\right)^{T} \phi\left(t_{k}\right)\right]^{2}
$$


where

$$
\begin{aligned}
\phi\left(t_{n}\right) & =\left[\sin \left(\omega t_{n}\right) \cos \left(\omega t_{n}\right) \sin \left(2 \omega t_{n}\right) \cos \left(2 \omega t_{n}\right)\right]^{T} \\
\theta\left(t_{n}\right) & =\left[a_{1}\left(t_{n}\right) b_{1}\left(t_{n}\right) a_{2}\left(t_{n}\right) b_{2}\left(t_{n}\right)\right]^{T}
\end{aligned}
$$

The weighing factor $\lambda^{n-k}$ clepends on a forgetting factor $\lambda$ and is added to allow the algorithm to track changes in the true parameter vector $\theta_{l}$. In this way the effect of previous measurements on the cost function $J\left(\theta, t_{n}\right)$ clecays exponentially. The recursive estimation of the parameter vector $\theta$ that minimizes the cost function $J\left(\theta, t_{n}\right)$ is given by Ljung and Söderström (1983) as

$$
\begin{aligned}
\hat{\theta}\left(t_{n}\right) & =\hat{\theta}\left(t_{n-1}\right)+L\left(t_{n}\right)\left[y\left(t_{n}\right)-\phi^{T}\left(t_{n}\right) \hat{\theta}\left(t_{n-1}\right)\right] \\
L\left(t_{n}\right) & =P\left(t_{n}\right) \phi\left(t_{n}\right) \\
P\left(t_{n}\right) & =\frac{1}{\lambda}\left[P\left(t_{n-1}\right)-\frac{P\left(t_{n-1}\right) \phi\left(t_{n}\right) \phi\left(t_{n}\right)^{T} P\left(t_{n-1}\right)}{\lambda\left(t_{n}\right)+\phi^{T}\left(t_{n}\right) P\left(t_{n-1}\right) \phi\left(t_{n}\right)}\right]
\end{aligned}
$$

The convergence of the parameter vector $\hat{\theta}$ towards the true parameter vector $\theta_{l}$ will depend on the statistical properties of the noise process $e\left(t_{n}\right)$ (Ljung and Söderström 1983). The estimation of $\theta_{i}$ using (3.4), (3.5) and (3.6) can be reduced to four separate estimations of $a_{1}, b_{1}, a_{2}$ and $b_{2}$, since sine and cosine are orthogonal functions, thus eliminating the computational efforts of manipulating 4-by-4 matrices.

The forgetting factor $\lambda$ of the least squares fitting algorithm determines the weight that is given to past measurements in the cost function (3.3). A value of $\lambda$ that is too high will give a high weight to past measurements making the convergence of $\hat{\theta}$ towards $\theta_{l}$ slow, whereas a value of $\lambda$ that is too low will make the estimations noise-sensitive. The final choice of $\lambda$ is given by this balance between tractability and noise-sensitivity. Model simulations using a known set of parameters $a_{k}$ and $b_{k}$ corrupted by white noise were used to obtain an optimum choice of $\lambda$. Based on these simulation studies a value of 0.95 was chosen for $\lambda$.

The estimated parameters $a_{k}$ and $b_{k}$ can be used to calculate the pulmonary features corrected for the modulation by the ventilator as

$$
y\left(t_{n}\right)-\sum_{k=1}^{2}\left(a_{k} \cos \left(\omega k t_{n}\right)+b_{k} \sin \left(\omega k t_{n}\right)\right)
$$

The features $y_{M P A P}, y_{s y s}, y_{d i a s}$ and $y_{d P / d t}$ are corrected using (3.7). The corrected features of $y_{u p}$ and $y_{\text {down }}$ are calculated from the corrected features of $y_{\text {sys }}$ and $y_{\text {dias }}$. The timing features of a beat, $y_{\text {period }}$ and $y_{\text {fall }}$, are 


\begin{tabular}{lccc}
\hline & $\begin{array}{c}\text { Initial } \\
\text { validation } \\
\text { boundaries }\end{array}$ & $\begin{array}{c}\text { Acceptable } \\
\text { range }\end{array}$ & $\begin{array}{c}\text { Validation } \\
\text { boundaries }\end{array}$ \\
\hline MPAP (mmHg) & 2 & $2-60$ & 1.10 \\
Systole (mmHg) & 2 & $5-70$ & 1.85 \\
Diastole (mmHg) & 2 & $1-50$ & 1.50 \\
Up pulse (mmHg) & 3 & $3-30$ & 1.95 \\
Down pulse (mmHg) & 3 & $3-30$ & 2.13 \\
$\left.d P / d t_{\text {max }}(\mathrm{mmHg} \mathrm{s})^{-1}\right)$ & 100 & $25-500$ & 23.8 \\
Period time (ms) & 120 & $20-200$ & 140 \\
Fall time (ms) & 120 & $15-180$ & 140 \\
\hline
\end{tabular}

Table 3.1: The initially used validation boundaries, the acceptable range of each feature and the validation boundaries as determined after evaluating the results of all 30 patients. The validation boundaries are the maximally allowed deviations of each feature from the corresponding feature of the beat model. MPAP, mean pulmonary artery pressure.

minimally modulated by the ventilator signal and do not require correction for the respiration artefact.

\section{Determination of end-expiratory feature values.}

One is also interested in the end-expiratory pressure values for the ventilated patient since here the condition of stable airway pressure is most likely to be met. Standard monitoring equipment approximates these endexpiratory values using a weighted filtering technique, which favours endexpiratory values over non-end-expiratory values (Hewlett Packard 1989). End-expiratory values are identified by analysing the beat-to-beat difference, assuming that the MPAP is relatively stable at end-expiration and relatively unstable elsewhere (Ellis 1985; Hewlett Packard 1989). Using the previously described estimation technique, however, the function describing the respiration artefact is known at all times and can be used to calculated the end-expiratory values with each new pulmonary pressure beat.

\subsubsection{Validation of pulmonary pressure}

The corrected features of a beat are used to add a validity flag to that beat. All pressure beats with their corrected features within an acceptable range are used to update a moving average model of each feature using

$$
y_{\text {model }}\left(t_{n}\right)=y_{\text {model }}\left(t_{n-1}\right) * 0.9+y\left(t_{n}\right) * 0.1
$$


The acceptable range is defined as the absolute minimum and maximum values of each feature see table 3.1. The factor 0.9 is chosen so that changes are tracked in time without tracking the ventilatory disturbances. The beat model now stores the history of the previous feature values. A beat is considered valid if its feature values are within the acceptable range and if they do not deviate too strongly from the previous feature values. In this way, a pressure beat with a sudden change in its shape will be rejected because it deviates from the beat model, whereas the beat model will slowly converge towards a new state if the change in shape is permanent. The maximally allowed deviation of the feature values from the beat model will be referred to as the validation boundaries. Initial estimates of the validation boundaries were used since no prior knowledge of the beat-tobeat difference was available (table 3.1). Both the acceptable range and the initial estimates of the validation boundaries were chosen after interviewing an experienced clinician and analysing results of a pilot study.

\subsection{Evaluation of performance}

The performance of the proposed filtering and validation technique was evaluated using data from thirty patients who were awaiting cardiac surgery. Informed patient consent was obtained the evening before surgery. The pulmonary artery pressure signal was obtained using a pressure transducer (Uniflow, Baxter) connected to a $7 \mathrm{~F}$ pulmonary artery catheter, which was introduced prior to surgery via the right jugular vein. The analog pressure signal was taken from the operating room monitoring system's (M1168A Component Monitoring System, HP) pressure modules (M1006A, $\mathrm{HP}$ ) which had a built-in lowpass filter with a cutoff frequency at 13 Hertz. The algorithms were implemented on an IBM-AT compatible personal computer. The analog pressure signal was imported at a sampling rate of 100 Hertz using an analog-to-digital convertor (DAS1600, Keithley Metrabyte) with an accuracy of 12 bits for $500 \mathrm{mmHg}$. Programming was done in Turbo Pascal vi.0 (Borland Pascal, Scotts Valley, CA). We designed a trend display that was updated every 5 seconds with the averaged MPAP feature. A 5-second epoch was considered valid if at least two periods were found to be valid.

During the surgical procedure the pulmonary artery pressure waveform and the 5-second trend display were observed by an experienced clinician who made notes of all relevant events. The validation algorithm correctly recognized pressure signals disturbed by coagulation, surgical manipula- 


\begin{tabular}{|c|c|c|c|}
\hline & \multicolumn{2}{|c|}{ Amplitude } & \multirow{2}{*}{$\begin{array}{l}\text { Corrections for } \\
\text { end-expiration }\end{array}$} \\
\hline & $1^{\text {st }}$ harmonic & $2^{\text {nd }}$ harmonic & \\
\hline$\overline{M P A P(\mathrm{mmHg})}$ & $1.89 \pm 0.54$ & $0.55 \pm 0.19$ & $1.66 \pm 0.45$ \\
\hline Systole (mmHg) & $1.86 \pm 0.63$ & $0.72 \pm 0.21$ & 0.54 \\
\hline Diastole (mmHg) & $2.21 \pm 0.65$ & $0.72 \pm 0.22$ & $1.92 \pm 0.51$ \\
\hline$d P / d t\left(\mathrm{mmHg} \mathrm{sec}^{-1}\right)$ & $10.9 \pm 4.1$ & $5.8 \pm 2.4$ & $1.9 \pm 0.5$ \\
\hline
\end{tabular}

Table 3.2: Results of the correction algorithm in 30 patients. The estimated amplitudes of the first and second harmonics and the corrections necessary to calculate the end-expiratory values from the corrected features are shown. All values are mean $\pm \mathrm{SD}$.

tions or flushes of the pressure line as invalid data and did not mark any 5 -second interval incorrectly as valid data. However, the system did mark successive 5 -second episodes as invalid which were considered valid by the clinician. This occurred only during atrial fibrillation; here the chosen validation boundaries of the timing features were too small. A representative example of the performance of the filtering algorithm during a stepwise change in ventilatory frequency is given in figure 3.2.

All feature values of each beat were stored on hard-disk for offline analysis. The average amplitudes of the first and second harmonic of each feature for all patients were calculated as $\sqrt{a_{1}^{2}+b_{1}^{2}}$ and $\sqrt{a_{2}^{2}+b_{2}^{2}}$ (table 3.2). Also shown in table 3.2 are the average values that were subtracted from each corrected feature to obtain its end-expiratory value. The average deviations of the feature values from the beat model have been calculated for all 30 patients and are given in table 3.3.

The percentage of valid periods, when the validation boundaries were chosen at $n$ times the average deviations found in all these patients, was calculated for $n$ ranging from 0.5 to 5 (figure 3.3). In general, the chance that invalid data are incorrectly identified as valid increases for values of $n$ that are too large, whereas for values of $n$ that are too low, insufficient periods will be marked as valid. Taking this disparity and the intended application (a closed-loop system) into consideration, we have chosen the validation boundaries for $n$ as 2.5 . With these new validation boundaries (table 3.1 ) $77 \pm 11 \%$ (mean $\pm \mathrm{SD}$ ) of the periods were found to be valid, while $85 \pm 13 \%$ of the 5 -second periods were valid. We also investigated whether the percentage of valid periods is biased on ventilator cycle. The ventilatory phase was extracted from the first harmonic of the MPAP feature. The percentage of valid periods was calculated for eight different. intervals in the ventilatory cycle (figure 3.4 ). 

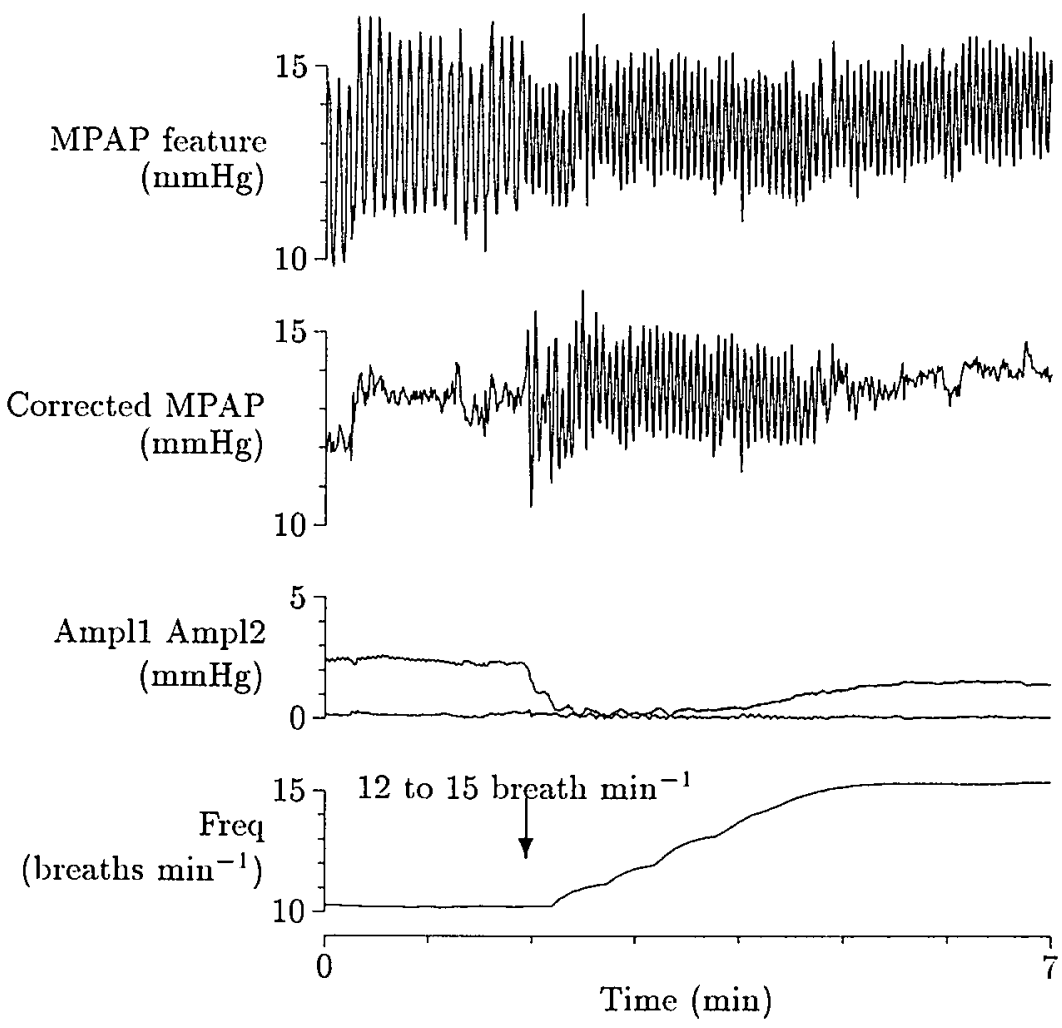

Figure 3.2: The performance of the correction algorithm for the mean pulmonary artery pressure (MPAP) feature during a step-wise change in ventilatory frequency. The top panel shows the fluctuation of the MPAP feature with the ventilatory frequency. The middle panels show the MPAP feature corrected for the respiration artefact and the amplitudes of the first (Ampl1) and second (Ampl2) harmonics as estimated by the algorithm. The lower panel shows the estimated ventilator frequency (Freq) which is increased step-wise from 12 to 15 breaths $\min ^{-1}$ at the arrow. It takes almost 3 minutes before the new ventilator frequency has been identified. During this period of time correction is not possible. 


\begin{tabular}{|c|c|c|c|}
\hline & Features & $\begin{array}{c}\text { Corrected } \\
\text { features }\end{array}$ & $\begin{array}{c}\text { Average } \\
\text { improvement }\end{array}$ \\
\hline$\triangle \mathrm{MPAP}(\mathrm{mmHg})$ & $1.39 \pm 0.33$ & $0.44 \pm 0.13$ & $68 \%$ \\
\hline$\Delta$ Systole (mmHg) & $1.56 \pm 0.43$ & $0.74 \pm 0.25$ & $53 \%$ \\
\hline$\Delta$ Diastole $(\mathrm{mmHg})$ & $1.68 \pm 0.41$ & $0.60 \pm 0.17$ & $64 \%$ \\
\hline$\Delta \mathrm{dP} / \mathrm{dt}\left(\mathrm{mmHg} \mathrm{s}^{-1}\right)$ & $13.2 \pm 4.9$ & $9.5 \pm 3.9$ & $28 \%$ \\
\hline$\Delta$ Up pulse (mrnHg) & $1.30 \pm 0.42$ & $0.78 \pm 0.31$ & $40 \%$ \\
\hline$\Delta$ Down pulse (mmHg) & $1.36 \pm 0.41$ & $0.85 \pm 0.30$ & $38 \%$ \\
\hline$\Delta$ Period time (ms) & $54.8 \pm 24.2$ & - & - \\
\hline$\Delta$ Fall time $(\mathrm{ms})$ & $56.5 \pm 22.1$ & - & - \\
\hline
\end{tabular}

Table 3.3: Reduction in the difference between the features of a beat and the corresponding features of the beat model, when the non-corrected and the corrected features of that beat were used. The average improvement is expressed as $\left(1-\Delta y_{c} / \Delta y\right) 100 \%$, where $\Delta y$ is the difference using the non-corrected and $\Delta y_{c}$ is the difference using the corrected feature.

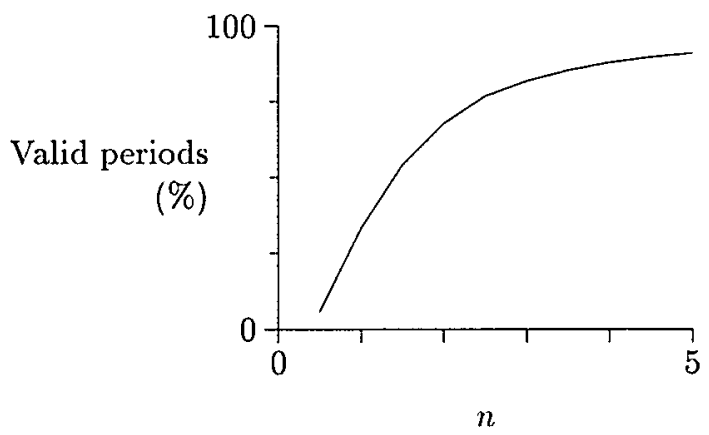

Figure 3.3: The percentage of valid periods when the validation boundaries are varied over $n$ times (from 0.5 till 5 in steps of 0.5 ) the average deviations from the beat model as found in 30 patients. 

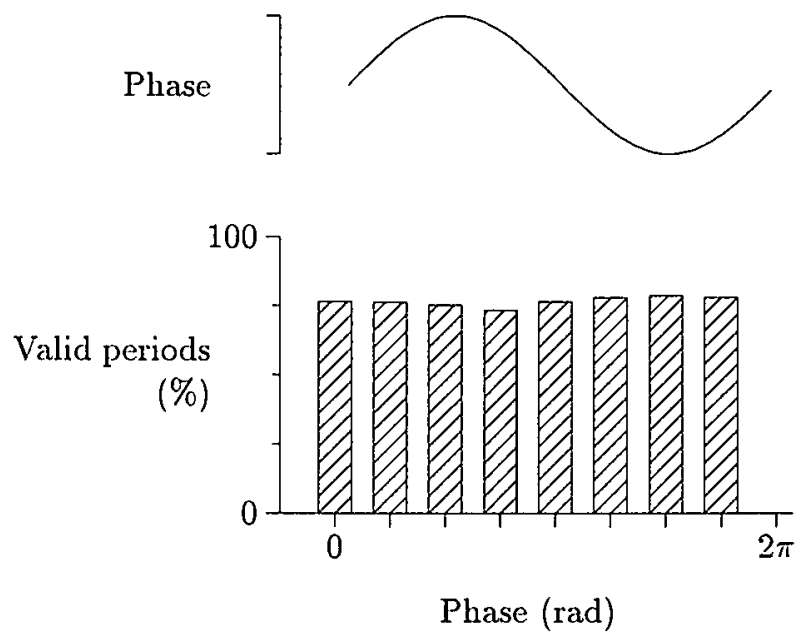

Figure 3.4: The percentage of valid periods as a function of the phase in the ventilator cycle. The current phase of the ventilator cycle was extracted using the first harmonic of the MPAP feature. The top panel schematically represents this harmonic, the lower panel represents the percentage of valid periods in each phase. The validation boundaries, that were determined after analysing the results of all 30 patients (table 3.1 ), were used. 


\subsection{Discussion}

The proposed algorithm provided a simple way to correct characteristic features of pulmonary pressure beats for the cyclic respiration artefact. We chose not to correct the pulmonary pressure waveform because this would require a time-consuming filtering technique. Pulmonary pressure beats were validated by comparing their corrected features with the averaged features of the previous pressure beats. The validation boundaries could be chosen sufficiently small because the noise caused by the respiration artefact had been reduced by the filtering algorithm (table 3.3). The algorithm identified an adequate percentage of pressure beats as valid to update a trend display every five seconds, while correctly recognizing pressure signals disturbed by coagulation, surgical manipulations or flushes of the pressure line as invalid data.

The proposed filtering algorithm can be seen as a sharp bandstop filter around the ventilator frequency and its higher harmonics. The sharpness of this bandstop filter is mainly determined by the forgetting factor $\lambda$ of the least squares fitting algorithm which allows the parameters $a_{k}$ and $b_{k}$ to create a time-varying phase and, thus, a frequency shift related to the firstorder time-derivative of this phase. The algorithm should not be mistaken for an ordinary bandstop filter, because a feature will only be corrected for the respiration artefact when it is consistently present in the pulmonary pressure signal; no correction will be made when the cyclic ventilator signal is not clearly present in the pulmonary pressure signal. In an alternative implementation, the time required for the algorithm to find the ventilator frequency in the pulmonary pressure signal (figure 3.2) can be eliminated by reading it directly from the ventilator.

We validated pulmonary pressure beats by analysing the fluctuations in eight characteristic features. Neural networks have been proposed to validate the shape of pressure signals (Odaka et al. 1994; Pike and Mustard 1992). Their disadvantages are that online learning is required and that their inference process is not as transparent. Online model identification (Ocasio et al. 1993) and spectral density estimation (Kuo and Chan 1993) have also been suggested to analyse the shape of pressure waveforms. Using these techniques, detection of invalid measurements will, however. be delayed because multiple pressure samples need to be evaluated before a change in the pressure waveform becomes apparent.

The proposed correction and validation algorithm may have several applications. It can be used to calculate end-expiratory values with each new pressure beat. The validity flag set by the algorithm can be used to filter out 
measurement artefacts in long-time trend recordings and can thus assist in the automated processing of pressure recordings. The corrected pulmonary features provide a smoother trend recording compared with algorithms that average pulmonary features over a fixed period of time. Finally, the validated pulmonary pressure can be used as a feedback signal in a closed-loop system where an artefact-free signal is essential. 


\section{Chapter 4}

\section{Detection of the dicrotic notch in arterial pressure signals}

S. A. A. P. Hoeksel

J. R. C. Jansen

J. A. Blom

J. J. Schreuder

Journal of Clinical Monitoring 13(5) 1997, 309-316. 


\section{Abstract}

Objective. A novel algorithm to detect the dicrotic notch in systemic arterial pressure signals is proposed. Its performance is evaluated using both aortic and radial artery pressure signals and its robustness to variations in design parameters is investigated.

Material and method. Most previously published dicrotic notch detection algorithms scan the arterial pressure waveform for the characteristic pressure change that is associated with the dicrotic notch. The aortic valve closure, however, is associated with the backwards motion of aortic blood volume. We developed an algorithm that uses arterial flow to detect the dicrotic notch in arterial pressure waveforms. Arterial flow is calculated from arterial pressure using simulation results with a three-element windkessel model. Aortic valve closure is detected after the systolic upstroke and at the minimum of the first negative dip in the calculated flow signal.

Results. In seven dogs ejection times were derived from a calculated aortic flow signal and from simultaneously measured aortic flow probe data. In all, 86 beats were analysed; the difference in ejection times was $-0.6 \pm 5.4 \mathrm{~ms}$ (mean \pm SD). The algorithm was further evaluated using 6 -second epochs of radial artery pressure data measured in 50 patients. Model simulations were carried out using both a linear windkessel model and a pressure and age dependent nonlinear windkessel model. Visual inspection by an experienced clinician confirmed that the algorithm correctly identified the dicrotic notch in $98 \%$ (49 out of 50 ) of the patients using the linear model and $96 \%$ (48 out of 50 ) of the patients using the nonlinear model. The position of the dicrotic notch appeared to be less sensitive to variations in algorithm's design parameters when a nonlinear windkessel model was used.

Conclusions. The detection of the dicrotic notch in arterial pressure signals is facilitated by first calculating the arterial flow waveform from arterial pressure and a model of arterial afterload. The method is robust and reduces the problem of detecting a dubious point in a decreasing pressure signal to the detection of a well-defined minimum in a derived signal.

Keywords signal processing • dicrotic notch • aortic input impedance • windkessel model $\bullet$ systolic time intervals 


\subsection{Introduction}

The position of the dicrotic notch in the arterial pressure signal conveys valuable information. Combined with electrocardiogram and phonocardiogram recordings it can be used to calculate systolic time intervals which in turn can be used to monitor cardiac function (van der Hoeven et al. 1977; Lewis et al. 1977). The pressure value at the dicrotic notch is well correlated with left ventricular end-systolic pressure (Dahlgren et al. 1991). Timing of the ejection phase is required in pulse contour methods (Jansen et al. 1990b) and is required for the control of beat-to-beat cardiac assist devices (Zelano et al. 1992). Application of the dicrotic notch to patient monitoring is still limited because it cannot always be easily derived from the arterial pressure waveform. The dicrotic notch is not always clearly visible and must be detected from a decreasing pressure signal. Also, reflected pressure waves and signal disturbances can cause pressure dips which are easily mistaken for the dicrotic notch.

Most notch-detection strategies try to identify the bending point around the dicrotic notch using some sort of first- or second-order time-derivative of the pressure signal (Starmer et al. 1973; Kinias et al. 1981; Gratz et al. 1992). Improved results can be obtained when these strategies are combined with rule-based logic (Oppenheim and Sittig 1995). Some methods minimize the chance of false detection by defining a time-window inside which the dicrotic notch should be located (Starmer et al. 1973; Gratz et al. 1992). An additional problem involves the real-time detection of the dicrotic notch which is often desired in the control of beat-to-beat cardiacassist devices. Sakamoto et al. (1995) used regression analysis to predict the occurrence of the dicrotic notch based on the time of the $R-R$ interval of the preceding period. A totally different approach was followed by $\mathrm{Ze}$ lano (1986) who used a filtering and peak-detection algorithm to extract the high-frequency components of the second heart sound from high-fidelity pressure recordings.

We evaluated the accuracy of a new dicrotic notch detection algorithm. The algorithm detects the dicrotic notch in the arterial pressure waveform by using an arterial flow signal that is calculated from arterial pressure and a simplified model of arterial afterload. The algorithm detects the notch almost instantly, is recursive, does not require high-fidelity pressure recordings and makes no a priori assumptions on the position of the dicrotic notch. Information derived from the dicrotic notch might be used for online monitoring of cardiac pump function. Furthermore, the position of the dicrotic notch might prove useful in further improving the artifact rejec- 
tion algorithms that are needed in closed-loop blood-pressure control systems. Both applications require an accurate and robust real-time detection technique because patient treatment is directly related to the information provided. After having verified the algorithm's correctness for aortic pressure signals, we evaluated the robustness of the detection algorithm using radial artery pressure signals, because in routine clinical practice arterial pressrues are often measured from peripheral sites.

\subsection{Algorithm}

Previously described dicrotic notch detection algorithms analyse the shape of the arterial pressure wave to cletect the dicrotic notch. The aortic valve closure, however, is associated with the retrograde motion of aortic blood volume. This would imply that the dicrotic notch can be detected more easily from the arterial flow wave than from the arterial pressure wave. We therefore developed a dicrotic notch detection algorithm that does not analyse the shape of the arterial pressure wave but of the arterial flow wave. Arterial flow can be estimated from arterial pressure if the impedance of the arterial tree is known.

\subsubsection{The windkessel model}

The three-element windkessel model has proved to be a good approximation of arterial afterload (Burkhoff et al. 1988). The windkessel approach (figure 4.1) models the impedance of the arterial tree by a characteristic input impedance, $Z_{a o}$, an arterial compliance, $C_{w}$, and a peripheral resistance, $R_{p}$. The characteristic input impedance represents the resistance seen directly from the heart. The arterial compliance models the ability of the aorta and arteries to store blood volume elastically. The peripheral resistance models the total resistance of the vascular bed.

The time-varying aortic flow, $I_{a o}$, can be calculated from aortic pressure, $P_{a o}$, using simulations with the windkessel model. During the systolic ejection phase aortic blood volume flows from the left ventricle into the windkessel compliance. The aortic valve closes as soon as aortic flow turns negative. The elasticity of the aortic valve causes a small negative dip in the aortic flow signal and the aortic valve is completely closed at the minimum of this dip (Jones and Sugawara 1993). During the diastolic phase aortic flow is assumed to be zero and the pressure over the windkessel compliance equals the aortic pressure. The windkessel compliance discharges through the peripheral resistance during both the ejection and the diastolic phase. 


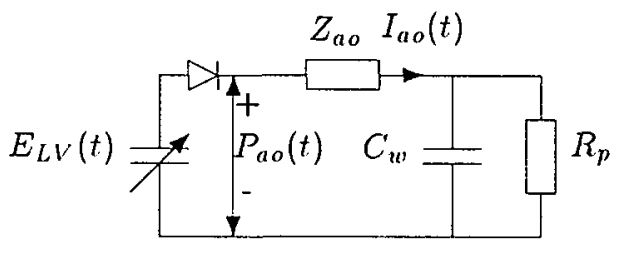

Figure 4.1: The elements of the windkessel model $\left(Z_{a o}, C_{w}\right.$ and $\left.R_{p}\right)$. The aortic valve is modelled by a diode which closes when aortic flow, $I_{a o}(t)$, turns negative. The left ventricle is modelled by a time varying elastance, $E_{L V}(t)$, (Sunagawa and Sagawa 1982).

The system's equation of the windkessel model can be found by recognizing that aortic flow, $I_{a o}$, equals the sum of flows through the windkessel compliance, $I_{C_{w}}$, and the peripheral resistance, $I_{R_{p}}$,

$$
\begin{aligned}
I_{a o} & =I_{R_{p}}+I_{C_{w}} \\
& =\frac{P_{C_{w}}}{R_{p}}+C_{w}^{\prime} \dot{P}_{C_{w}}
\end{aligned}
$$

where $P_{C_{w}}$ is the pressure over the windkessel compliance and $\dot{P}_{C_{w}}$ is its time-derivative. $P_{C_{w}}$ is also equal to the aortic pressure minus the pressure drop across the characteristic input impedance,

$$
P_{C_{w}}=P_{a o}-Z_{a o} I_{a o}
$$

Substitution of (4.2) in (4.1) yields the relation between aortic flow and aortic pressure,

$$
\left(1+\frac{Z_{a o}}{R_{p}}\right) I_{a o}+Z_{a o} C_{w} \dot{I}_{a o}=\frac{P_{a o}}{R_{p}}+C_{w} \dot{P}_{a \circ}
$$

The differential equation (4.3) can be approximated in the discrete time domain using the bilinear transformation (Oppenheim et al. 1983).

\subsubsection{Choice of windkessel model parameters}

The values of the three model parameters $\left(Z_{a o}, R_{p}\right.$ and $\left.C_{w}\right)$ are needed to describe the windkessel model's response fully. For this application, however, we are only interested in the behaviour of the uncalibrated arterial flow waveform at end-ejection. We therefore only require knowledge of the 
two time constants of the windkessel model $Z_{a o} C_{w}$ and $R_{p} C_{w}$. Estimates of $Z_{a o}$ and $C_{w}$ can be taken from previously published studies (Liu et al. 1986; Latson et al. 1988; Laskey et al. 1990). Given $Z_{a o} C_{w}, R_{p} C_{w}$ can be estimated by assuming that aortic flow is zero during diastole. The exponential pressure decay during diastole is generally used for this purpose. This technique, however, fails in our application since we do not yet know where the diastolic phase begins. The end of the diastolic phase can however be detected from the arterial pressure signal and the calculated end-diastolic flow value can thus be used to update $R_{p}$ recursively using

$$
R_{p}(n+1)=R_{p}(n)+\alpha I_{\text {dias }}(n)
$$

where $I_{\text {dias }}(n)$ is the end-diastolic flow value of the $n^{\text {th }}$ beat and $\alpha$ is a filter parameter which is chosen such that the true peripheral resistance value is tracked in time and the beat-to-beat variations in the estimated $R_{p}$ are minimized. We found that a value for $\alpha$ of $0.04 \mathrm{mmHg} \mathrm{m}^{-2} \mathrm{~s}^{2}$ works well.

\subsubsection{Nonlinear windkessel model}

The simulations with the windkessel model can be improved upon when the changing visco-elastic properties of the arterial system with patient age, gender and pressure are taken into account. In general, the arterial system gets stiffer with higher age and at higher pressure levels ( $\mathrm{Li}$ et al. 1990; Langewouters et al. 1984). Langewouters et al. (1984) developed an arctangent pressure-area relationship which incorporates these dependencies. Using this arctangent pressure-area relationship the values of the characteristic input impedance and the windkessel compliance can be corrected for age, gender and arterial pressure level (Wesseling et al. 1993).

\subsubsection{The algorithm}

The algorithm detects the different pressure periods in the arterial pressure signal by evaluating upstroke pressures (chapter 3 ). An upstroke pressure is detected at each local maximum in the arterial pressure signal and its value is defined as the pressure difference between the last local minimum and the current maximum. An upstroke is identified as the systolic upstroke if its value is greater than $15 \mathrm{mmHg}$. Diastolic and systolic pressures are the minimum and maximum pressure values of the systolic upstroke. For the linear windkessel model the time-varying aortic flow is calculated from the arterial pressure signal using equation 4.3. For the nonlinear case, aortic flow is calculated in a similar fashion, but age, gender and aortic pressure 
values are used to calculate the windkessel model parameters (Langewouters et al. 1984; Wesseling et al. 1993). The dicrotic notch is detected at the first local minimum in the aortic flow signal after the aortic flow turned negative. The peripheral resistance is updated with each newly detected beat using equation 4.4. In pseudo-code, the algorithm can be given as follows:

repeat

repeat until $\min \left\{P_{a o}(t)\right\}$

$I_{\text {dias }}=I_{a o}(t)$

repeat until $\max \left\{P_{a o}(t)\right\}$

if upstroke $>$ minimal_upstroke then begin

detect_systolic_upstroke

$R_{p}(n+1)=R_{p}(n)+\alpha I_{\text {dias }}$

repeat until $I_{a o}(t)<0$ and $\min \left\{I_{a o}(t)\right\}$

detect_dicrotic_notch

end

until stop

The functions $\min \{\ldots\}$ and $\max \{\ldots\}$ return true when their argument reaches a local minimum or maximum. Local minima and maxima in pressure and flow signals are evaluated using the first-order time-derivatives of the appropriate signals.

\subsection{Evaluation of performance}

The performance of the proposed algorithm was tested in two ways: In an animal study the correctness of the underlying idea, i.e. using a calculated flow signal to detect the dicrotic notch, was verified. Next the algorithm's robustness to a mismatch in windkessel model parameters was evaluated using a set of human radial artery pressure signals. We used radial artery pressure signals because they are routinely measured in a clinical setting and because the reflections that are observed in the radial artery pressure pulse would truly challenge the detection algorithm.

Before analysis, the arterial pressure signals were digitally filtered using a third-order lowpass Butterworth filter with its cutoff frequency at 15 Hertz. This filter provides a smooth pressure signal and avoids the algorithm falsely labelling a signal disturbance as a local minimum or maximum. Data were analysed offline, using the above described algorithm implemented in Matlab (Matlab v4.2b, The MathWorks Inc., Natick, MA). 


\subsubsection{Comparison with simultaneously measured flow probe data}

Simultaneously measured aortic flow and pressure signals were obtained from seven anaesthetized open chest dogs. Data were taken from the baseline measurements of another study protocol. The protocol was approved by the animal ethics committee of the Maastricht University. The weight of the dogs varied between 24 and $38 \mathrm{~kg}$, average $30 \mathrm{~kg}$. An electro-magnetic flow probe (Skalar, Delft, the Netherlands) was placed around the ascending thoracic aorta. A micro-tip catheter (Sentron, Rhoden, the Netherlands) was introduced via the left femoral artery and positioned close to the aortic flow probe. From each dog a 5-second interval of raw data was taken using an analog-to-digital converter (DAS1600, Keithley Metrabyte) and stored on the hard disk of an IBM-AT compatible personal computer at a sampling rate of $200 \mathrm{Hertz}$ and an accuracy of 12 bits for $500 \mathrm{mmHg}$.

The stored signals were analysed offline. The flow probe signal was digitally lowpass filtered using a third-order Butterworth filter to compensate for the time clelay in the pressure signal caused by using the same filter in the dicrotic notch detection algorithm. The start and end of the ejection phases were determined manually in the filtered flow probe signal by an experienced clinician. The previously described algorithm was used to detect the start and end of the ejection phases in the aortic pressure signal. The numeric values of the windkessel model parameters were taken over from a previously published dog study (Latson et al. 1988). $Z_{a o}$ was chosen as $0.162 \mathrm{mmHg} \mathrm{ml}^{-1} \mathrm{~s}$ and $C_{w}$ as $0.361 \mathrm{ml} \mathrm{mmHg}^{-1}$. Each 5 -second interval was processed 10 times before analysis to allow iteration of the peripheral resistance parameter $R_{p}$ (according to equation 4.4). Between 11 and 14 beats were analysed for each dog, on average 12.3 beats. The seven dogs had an heart rate of $170 \pm 15$ (mean \pm SD) beats $\mathrm{min}^{-1}$ and their systolic ejection time was $175 \pm 23 \mathrm{~ms}$. The difference in time between pressure and flow probe signals in all 86 beats was $6.4 \pm 4.0 \mathrm{~ms}$ at the start of the ejection phase and $5.8 \pm 4.6 \mathrm{~ms}$ at the end of the ejection phase. The difference in ejection times derived from both signals was $-0.6 \pm 5.4 \mathrm{~ms}$.

\subsubsection{Sensitivity to variations in windkessel model parame- ters}

The robustness of the algorithm was analysed using radial artery pressure signals of 50 patients who were catheterized before undergoing cardiac surgery. Patients' weight varied between 51 and $130 \mathrm{~kg}$, average $76 \mathrm{~kg}$. Most patients, 29 out of 50, were male. Patients' were aged between 40 
and 80 years, average 64 years. All patients gave informed consent for use of their, routinely measured, data. Haemodynamic measurements were obtained after induction of anaesthesia and prior to the start of the operation. The pressure signal was obtained using a saline-filled radial artery cannula (20-gauge) and a pressure transducer (Uniflow, Baxter) with a built-in lowpass filter with a cutoff frequency at $13 \mathrm{Hertz}$. The analog pressure signal was sampled at a rate of $100 \mathrm{Hertz}$ and with an accuracy of 12 bits for $500 \mathrm{mmHg}$ using an analog-to-digital converter (DAS1600, Keithley Metrabyte). For each patient 6 seconds of radial artery pressure data were analysed offline using both a linear and a nonlinear windkessel model.

The model parameters of the linear windkessel model were based on a previously published study by Laskey et al. (1990). $Z_{a o}$ was chosen as $0.061 \mathrm{mmHg} \mathrm{ml}^{-1} \mathrm{~s}$ and $C_{w}$ as $1.99 \mathrm{ml} \mathrm{mmHg}^{-1}$. Each 6-second episode was replayed up to 10 times to allow for iteration of $R_{p}$ (according to equation 4.4). Visual inspection by an experienced clinician revealed that the algorithm correctly identified the dicrotic notch in all pressure periods of $98 \%$ ( 49 out of 50 ) of the patients; see figure 4.2 for some illustrative examples. In one patient, the clinician did not agree with the placement of the dicrotic notch by the algorithm; see figure 4.3. Subsequently, the second beat of each 6-second episode was analysed for all patients. These 50 pressure beats had an average duration of $939 \pm 180 \mathrm{~ms}$ and an average ejection time of $350 \pm 40 \mathrm{~ms}$. The sensitivity to a mismatch in windkessel model parameters was investigated by evaluating the shift of the dicrotic notch time when the time constant $Z_{a o} C_{w}$ was taken at $n$ times the control value. The multiplier $n$ ranged from 0.25 to 2 , which covers the ranges reported elsewhere (Liu et al. 1986; Laskey et al. 1990). The shifts in dicrotic notch times are shown in figure 4.4. The placement of the dicrotic notch by the algorithm was rejected when it deviated by $100 \mathrm{~ms}$ or more from its control value (i.e. for $n$ as 1 ). These rejected values were not used in calculating the mean shift. The total number of rejected values is indicated in figure 4.4. For these rejected values, the algorithm generally incorrectly labelled reflections in the pressure signals as the dicrotic notch for too low values of $n$, whereas for too high values of $n$ the calculated aortic flow did not turn negative at the dicrotic notch.

We also investigated whether better results are obtained using the nonlinear windkessel model. Aortic flow was calculated from arterial pressure using the method described by Wesseling et al. (1993). Visual inspection of all 6 -second episodes by an experienced clinician confirmed the correctness of the algorithm's decisions in $96 \%$ (48 out of 50) of the patients. Again the algorithm failed to identify correctly the dicrotic notches in the 

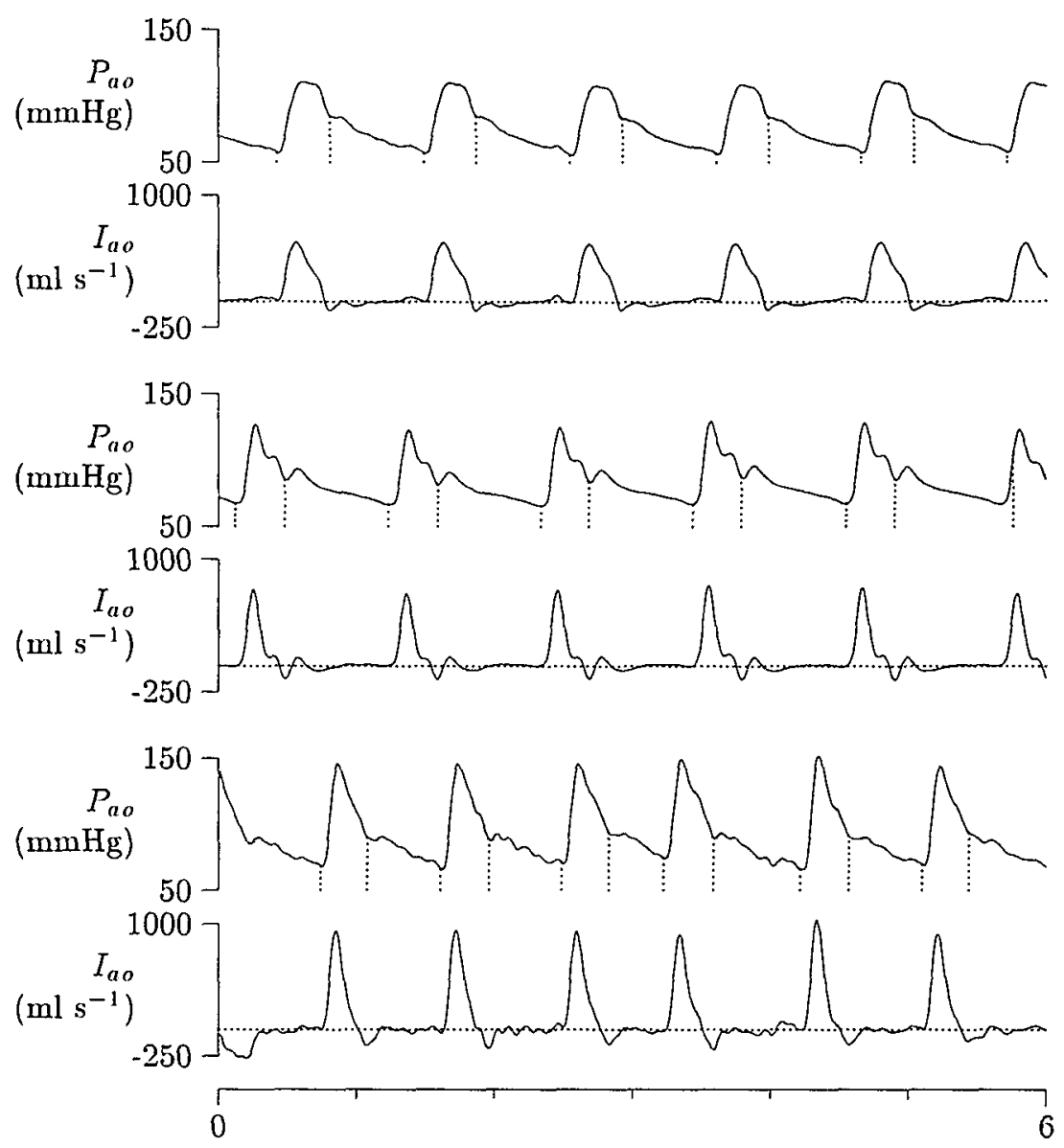

Time (s)

Figure 4.2: Illustrative examples of the dicrotic notch detection algorithm in 3 patients. Shown are the measured aortic pressure, Pao, and the calculated aortic Hlow, Iao. Top panels are of a 79-year-old male, middle panels of a 50-year-old male and lower panels of a 54-year-old female. A linear windkessel model is used $\left(Z_{a o} C_{w}=0.12 \mathrm{~s}\right)$. 


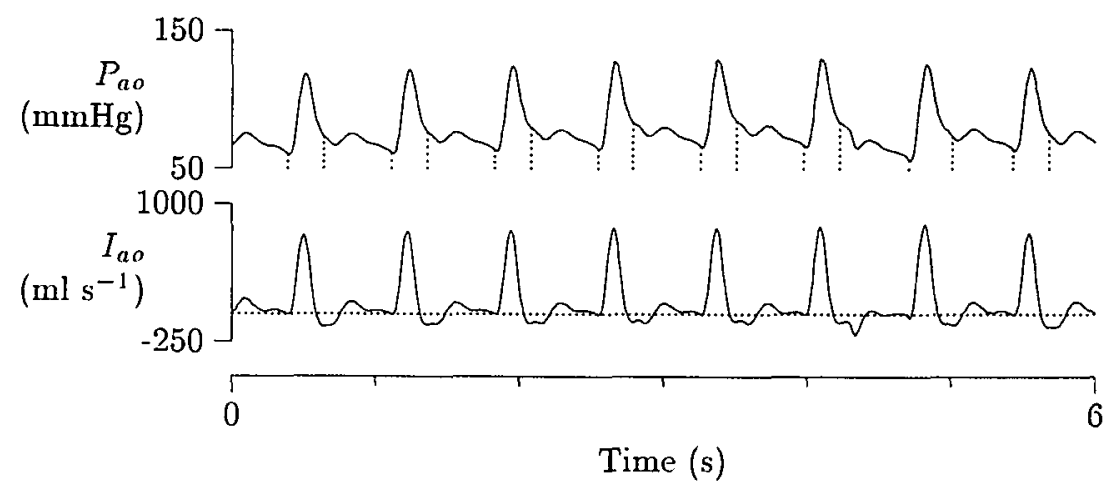

Figure 4.3: Pressure signal of a 52-year-old male patient where both the linear and the nonlinear windkessel model failed to correctly identify the dicrotic notch. The measured aortic pressure, $P_{a o}$, and the calculated aortic flow, $I_{a o}$, are shown. In total the linear model incorrectly identified the dicrotic notch in $2 \%$ ( 1 out of 50 ) of the patients and the nonlinear in $4 \%$ ( 2 out of 50 ) of the patients. In this example, aortic flow is calculated using the linear windkessel model $\left(Z_{a o} C_{w}=0.12\right.$ s).

pressure signal that is shown in figure 4.3. We also tested the robustness of the algorithm to a variation in model parameters. The second beat of each 6 -second episode was analysed when the assigned patient age was varied in steps of 5 years, ranging from -20 to 20 years (see figure 4.5 ). Results for two patients were rejected because the placement of the dicrotic notch deviated by $100 \mathrm{~ms}$ or more when the patient age was varied; this is indicated in figure 4.5. These were the same two patients about whom the clinician and algorithm disagreed in the control situation.

\subsection{Discussion}

In seven dogs the feasibility of the underlying idea, i.e. the application of a calculated aortic flow signal to detect the dicrotic notch in an arterial pressure signal, was investigated. The difference in duration of ejection time as derived from arterial pressure and a simultaneously measured flow probe signal $(-0.6 \pm 5.4 \mathrm{~ms})$ was within one sample time of the analog-to-digital converter $(5 \mathrm{~ms})$. The larger difference in timing of start $(6.4 \pm 4.0 \mathrm{~ms})$ and end $(5.8 \pm 4.6 \mathrm{~ms})$ of the ejection phase might be caused by a systematic difference in the position of the pressure sensor with respect to the aortic flow probe. In the radial artery pressure signals of 50 patients, the algorithm 


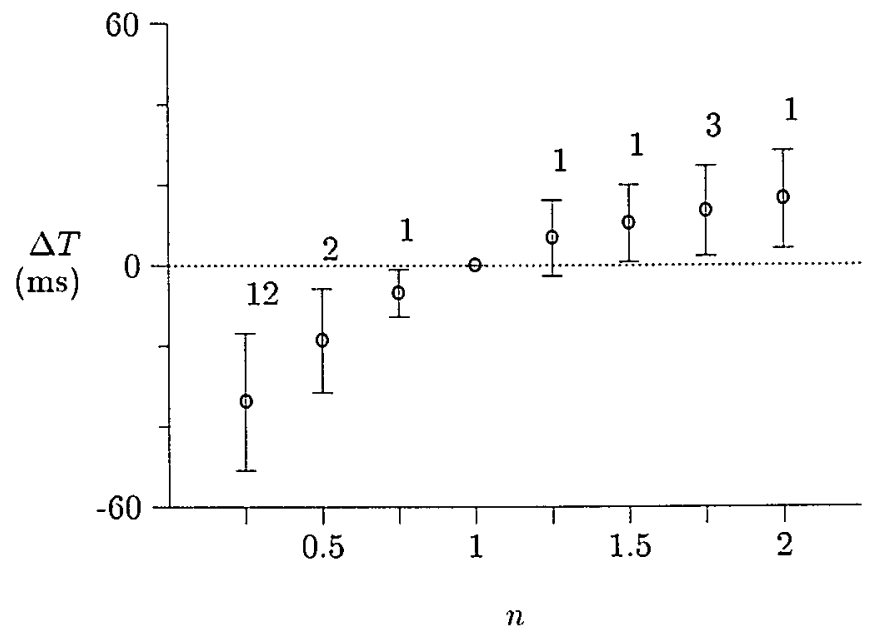

Figure 4.4: The shift in dicrotic notch times $(\Delta T)$ in all 50 patients when the time constant $Z_{a o} C_{w}$ was taken at $n$ times the control value. For each patient the second pressure period was analysed. All dicrotic notch times were compared with control (i.e. for $Z_{a o} C_{w}$ as $0.12 \mathrm{~s}$ ). Shown are means $\pm \mathrm{SD}$. The values inside the figure indicate the number of periods where the position of the dicrotic notch deviated by $100 \mathrm{~ms}$ or more from control. These values were not used in calculating the average shift and SD. 


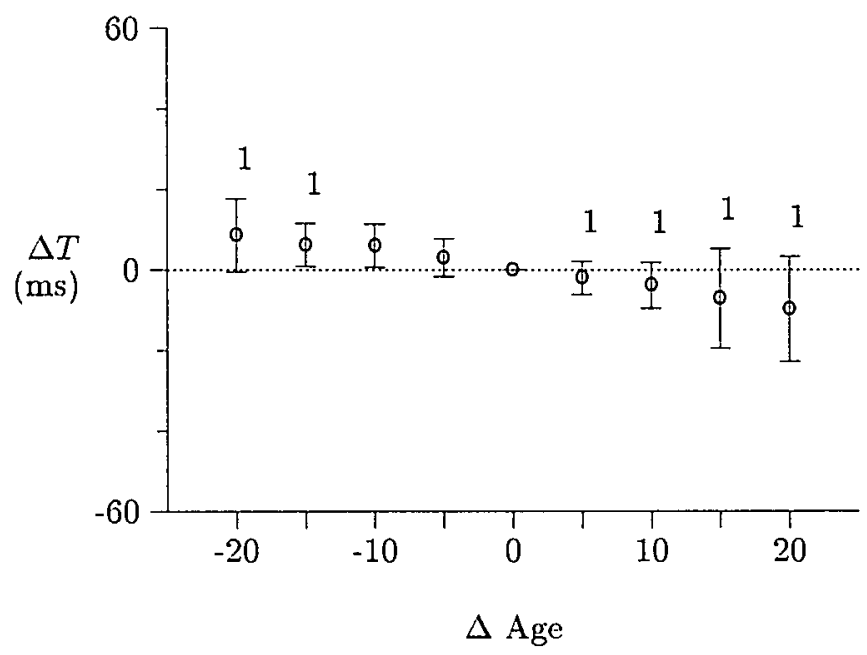

Figure 4.5: The shift in dicrotic notch times $(\Delta T)$ in all 50 patients when the assigned patient age in the nonlinear windkessel model was varied. For each patient the second pressure period was analysed. All dicrotic notch times were compared with control (i.e. for $\Delta$ Age as 0 ). Shown are means $\pm S D$. The values inside the figure inclicate the number of periods where the position of the dicrotic notch deviated by $100 \mathrm{~ms}$ or more from control. These values were not used in calculating the average shift and SD. 
placed markers at the start and end of the ejection phase. The positions of these markers were visually inspected by an experienced clinician. The placement of the dicrotic notch by the algorithm was questionable in $2 \%$ ( 1 out of 50 ) and $4 \%$ ( 2 out of 50 ) of the patients when a linear and a nonlinear windkessel model were used respectively (see figure 4.3).

We used radial artery pressure signals in our evaluation of the algorithm's robustness because they are routinely monitored in a clinical situation. The radial artery is readily accessible and relatively easy to cannulate. Compared with aortic pressures, radial artery pressures are distorted by reflected pressure waves and by changes in visco-elastic properties over the arterial tree. As a result, pressure and flow signals measured more distally have sharper slopes, higher peak values and a more oscillatory behaviour (Nichols et al. 1990). The derived duration of the left ventricular ejection phase remains fairly constant over the arterial tree, though the transmission of the arterial pulse could introduce deviations when it is calculated from more distally measured sites (Lewis et al. 1977; Nichols et al. 1990). Schwid et al. (1987) argue that, especially in stiff arteries, this may prevent accurate derivation of aortic valve closure from the radial artery pressure waveform. Based on simulation results with a simplified model of the arterial tree they state that the radial artery dicrotic notch is predominantly dependent on arterial wall properties, rather than on the closure of the aortic valves. This may partly explain the discrepancy between the clinician's and the algorithm's positioning of the dicrotic notches in two of the 50 patients. The delay that is introduced by the transmission of the pressure pulse through the arterial system makes peripheral pressures less than ideal for the beat-to-beat control of left ventricular assist devices (Zelano 1986). For analysis of systolic time intervals from peripheral pressures, additional information from phonocardiogram recordings is needed (Lewis et al. 1977).

The validity of the windkessel model itself might also be questioned. The validity is determined by the approximation of arterial afterload by a three-element windkessel model and by the fit of the windkessel model parameters to the true patient's response. The windkessel model has successfully been used to model arterial afterload using both aortic (Burkhoff et al. 1988) and radial artery pressures (Wesseling et al. 1993), though we should bear in mind that it does not explain the changes in the pressure waveform over the arterial tree. Accurate calibration of the windkessel parameters is not necessary in this application, since we only require knowledge of relative flow values. In the linear case, the mismatch between the patient parameter values and the model parameter values of $Z_{a o}$ and $C_{w}$ may be large, but 
the mismatch in $Z_{a o} C_{w}$ will be limited since $Z_{a o}$ is inversely related to the square root of $C_{w}$ (Laskey et al. 1990). In the nonlinear case, the accuracy of the model simulations is influenced to a large extent by the estimation of the maximum cross-sectional area of the aorta which does not correlate well with patient age and gender (Langewouters et al. 1984). But again this parameter is not required here, because the absolute flow values are not needed.

The robustness of the algorithm was analysed by evaluating the effect of a mismatch in model parameters on the placement of the dicrotic notch. The variations used in $Z_{a o} C_{w}$ approximately cover the ranges described elsewhere (Liu et al. 1986; Laskey et al. 1990). The algorithm failed to detect the dicrotic notch in several patients when $Z_{a o} C_{w}$ was varied too much (figure 4.4). One might therefore consider use of a simple identification routine to get a coarse estimation of $Z_{a o} C_{w}$ (Ocasio et al. 1993). But it might also be questioned whether intervidual variations in $Z_{a o} C_{w}$ are truly so large, since the algorithm correctly identified the dicrotic notch in $98 \%$ (49 out of 50 ) of the patients with one fixed value for $Z_{c o} C_{w}(0.12 \mathrm{~s})$. The algorithm appeared to be less sensitive to variations in algorithm design parameters when the changes in visco-elastic properties with patient age, gender and arterial pressure were taken into account. This was to be expected since the nonlinear windkessel model incorporates more a priori knowledge and can therefore better model the true patient response. Detection of the dicrotic notch is robust enough to use the derived duration of the left ventricular ejection phase in pulse contour methods (Jansen et al. 1990b; Wesseling et al. 1993) and as an additional feature in pressure validation routines (see chapter 5 ).

In conclusion, the detection of dicrotic notches by the algorithm was accurate for radial artery pressure signals and highly accurate for aortic pressure signals. The placement of the dicrotic notch was minimally influenced by variations in windkessel model parameters. When windkessel model parameters were varied too strongly, the algorithm either incorrectly labelled reflections as the dicrotic notch or the flow waveform did not turn negative at the dicrotic notch. The algorithm is intuitively appealing because it uses a physiological basis (the arterial flow waveform) to detect the dicrotic notch and because it reduces the problem of detecting a dubious point in a decreasing pressure signal to the detection of a well-defined minimum in a derived signal. Future research should concentrate on the extent to which the information from the dicrotic notch can be used as an online monitoring parameter and whether the position of the dicrotic notch can be used as an additional feature in the pressure validation routines that are 
needed in closed-loop blood-pressure control systems. 


\title{
Chapter 5
}

\section{Validation of irregular blood-pressure signals}

\author{
S. A. A. P. Hoeksel
}

J. R. C. Jansen

J. A. Blom

J. J. Schreuder 


\section{Abstract}

Objective. To investigate whether timing of left and right ventricular ejection, as derived from arterial and pulmonary artery blood-pressure signals, can be used to design a signal validation algorithm for pressure beats at an irregular heart rate.

Material and method. Pressure beats were detected simultaneously in arterial and pulmonary pressure signals by correlating the derivatives of both signals. Start and finish of left and right ventricular ejection were detected in the arterial and pulmonary pressure beats using a previously described filtering technique. Three validation features were calculated for each pair of arterial and pulmonary pressure beats; the absolute difference between the duration of ejection and its average for the (1) left and (2) right ventricle and (3) the absolute difference between the durations of left and right ventricular ejection. A pair of pressure beats was rejected if at least one of its validation feature values was too large. The algorithm was experimentally applied to radial and pulmonary artery blood-pressure data of 14 patients with an irregular heart rate.

Results. In each patient between 70 and 180 (on average 121) pairs of pressure beats were analysed. The average values of the validation features derived from the durations of left and right ventricular ejection and the difference between these durations were $22.3,37.8$ and $51.9 \mathrm{~ms}$, respectively. A validation routine based on these average values interpreted between 55 and $97 \%$ (average $85 \%$ ) of the beats as valid.

Conclusions. Pressure beats in blood-pressure signals at an irregular rate can adequately be detected when arterial and pulmonary pressures are analysed simultaneously. The beat-to-beat fluctuations in left and right ventricular ejection times appeared to be small enough for the information derived from them to be used as validation criteria for beats at an irregular rate.

Keywords atrial fibrilation $\bullet$ signal validation $\bullet$ ejection time $\bullet$ irregular heart rate 


\subsection{Introduction}

Closed-loop blood-pressure controllers require signal validation algorithms to prevent the closed-loop system from responding to disturbances in the measured pressure signal. Discrepancies between measured and true pressure are introduced for example by flushing of the catheter or electrical interference with the coagulation equipment. Validation algorithms for arterial pressure signals extract a set of characteristic features from each pressure beat and reject those beats whose feature values exceed a preset limit or deviate too strongly from the average feature values of previous pressure beats (Blom 1990). By first correcting the features for the cyclic respiration artefact, pulmonary artery pressure signals can be validated in a similar manner (chapter 3). These techniques fail however for pressure beats at an irregular rate owing to the large beat-to-beat fluctuations in the features.

We were unable to trace any techniques that had been developed for the validation of blood-pressures signals at an irregular rate. Development of validation techniques for patients with irregular heart rates caused by, for example, atrial fibrilation is however needed as this group of haemodynamically unstable patients would chiefly benefit from closed-loop control of haemodynamics during and shortly after cardiac surgery. Because both ventricles contract nearly simultaneously, we speculated that timing of left and right ventricular ejection might prove useful for designing validation criteria for pressure beats at an irregular rate. A validation algorithm was developed and evaluated using radial and pulmonary artery blood-pressure signals because these pressures are routinely monitored in clinical practice.

\subsection{Algorithm}

Generally speaking, the validation algorithm can be divided into three parts. In the first part, the start of each pressure beat is detected by correlating arterial and pulmonary pressure signals. In the second part, the end of left and right ventricular ejection is identified from the arterial and pulmonary pressure signals. In the third part, validation features are introduced and each pair of arterial and pulmonary pressure beats is validated by analysing the fluctuations in these validation features. 

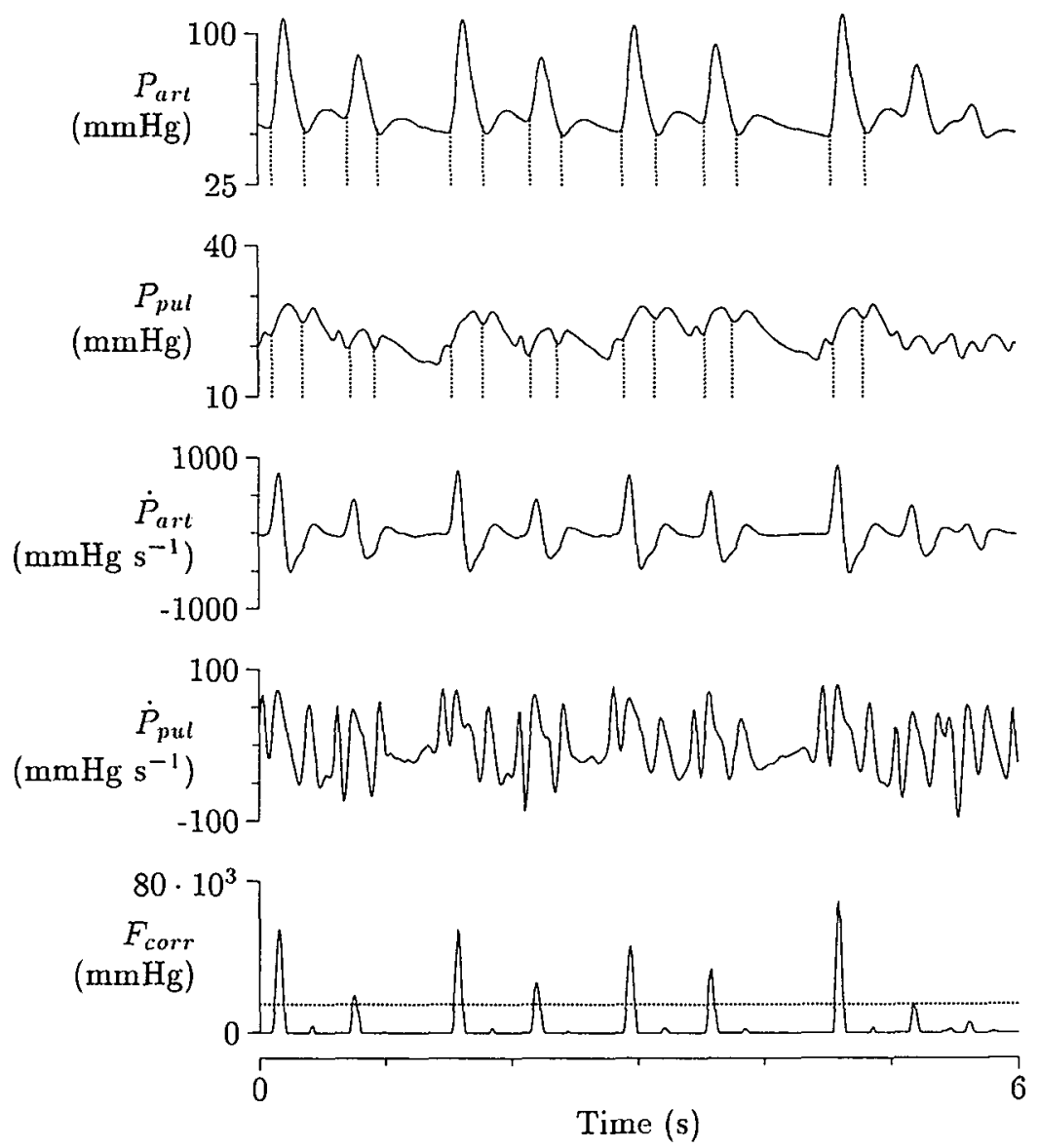

Figure 5.1: Illustrative example of the detection of systolic upstrokes in radial, $P_{a r t}$, and pulmonary artery pressure signals, $P_{p u l}$. $F_{c o r r}$ describes a correlation between the time derivatives of the arterial and pulmonary pressure signals (equation 5.1). In this example the assumed time delay is set at $t_{d}=0 \mathrm{~ms}$. Systolic upstrokes are detected when $F_{\text {corr }}$ crosses the detection level (dotted line). Also shown are the derivatives of $P_{a r t}$ and $P_{p u l}$. See text for further explanations. 


\subsubsection{Detection of systolic upstrokes}

Detection of systolic upstrokes, i.e. the fast increase in pressure at the start of ejection, is complicated in blood-pressure signals at an irregular rate owing to the impossibility of defining a narrow time window inside which systolic upstrokes can be detected. Moreover, a fixed detection level for a pressure signal or its derivative will fail to detect all systolic upstrokes and may incorrectly label reflections in the pressure signal as systolic upstrokes. An additional problem is the dip in the pulmonary pressure signal that is often observed at end diastole (figure 3.1, page 30 ) and acts as an ambiguous start of ejection. Systolic upstrokes in arterial and pulmonary pressure signals are however closely related in time, because both ventricles contract nearly simultaneously. Based on this we detected systolic upstrokes simultaneously in the arterial and pulmonary pressure signal.

Upstroke pressures are clearly visible in the time derivative and therefore we defined for our purposes a correlation factor between the time derivatives of the arterial pressure signal, $P_{\text {art }}$, and the pulmonary artery pressure signal, $P_{p u l}$, as

$$
F_{\text {corr }}\left(t, t_{d}\right)=\left\{\begin{array}{ll}
\dot{P}_{a r l}(t) \dot{P}_{p u l}\left(t-t_{d}\right) & \begin{array}{l}
\dot{P}_{a r l}(t)>0 \\
\dot{P}_{p u l}\left(t-t_{d}\right)>0
\end{array} \\
0 & \text { elsewhere }
\end{array}\right. \text { and }
$$

where the dot indicates the time derivative and $t_{d}$ models an unknown time shift between the arterial and pulmonary pressure waveform related to the site of pressure recording. A pair of pressure beats was detected when $F_{\text {corr }}$ crossed a detection level, $F_{l / h}$. The optimum value of $F_{t h}$ depends on the maximum values of $\dot{P}_{a r t}$ and $\dot{P}_{p u l}$ during the systolic upstroke and will therefore vary between patients. The variables $t_{d}$ and $F_{l h}$ therefore need to be determined experimentally for each patient. The start of left and right ventricular ejection was detected by backtracking in time from the threshold crossing to the first local minimum in $P_{a r t}$ and $P_{p u l}$ respectively. Systolic pressure was defined as the first local maximum in the pressure signal after the start of ejection. The proposed detection method is illustrated in figure 5.1 .

The arterial pressure signal is routinely measured at peripheral sites. The transmission of the arterial pressure over the arterial tree will therefore introduce a time shift between arterial and pulmonary pressure signals. Additional delays are introduced by the different properties of the fluidfilled measuring systems. To find the optimum value of $t_{d}$ for a given 


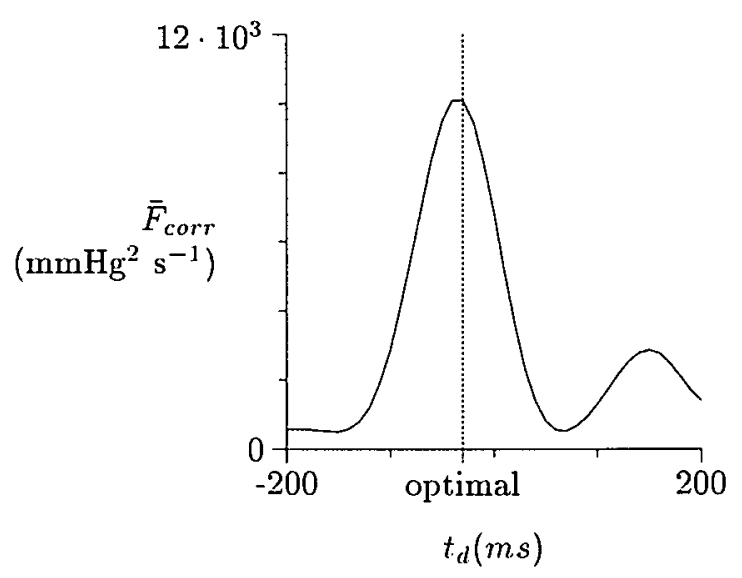

Figure 5.2: The changes in $\bar{F}_{\text {corr }}$ when the time shift, $t_{d}$, between arterial and pulmonary pressure signals is varied from -200 to $200 \mathrm{~ms}$ in steps of $10 \mathrm{~ms}$. The time shift is optimum for the maximum value of $\bar{F}_{\text {corr }}$ at $t_{d}=-30 \mathrm{~ms}$.

patient, we evaluated

$$
\bar{F}_{\text {corr }}\left(t_{d}\right)=\frac{1}{T} \int_{0}^{T} F_{\text {corr }}\left(t, t_{d}\right) d t
$$

for $t_{d}$ ranging from -200 to $200 \mathrm{~ms}$. The time $T$ denotes the duration of the measurement period considered. The optimum value of $t_{d}$ was defined as the value that maximized $\bar{F}_{\text {corr }}$ in equation 5.2 (figure 5.2).

\subsubsection{Detection of the dicrotic notch}

The end of left and right ventricular ejection was detected at the dicrotic notch in respectively the arterial and pulmonary pressure signal. For the arterial pressure signal the dicrotic notch was detected from the shape of the modelled arterial flow curve (chapter 4). Model simulations with a three-element windkessel model were used to calculate the arterial flow waveform from $P_{\text {art }}$. The windkessel model (figure 4.1, page 45) describes the impedance of the arterial tree by a characteristic input impedance, $Z$, a windkessel compliance, $C_{w}$ and a peripheral resistance, $R_{p}$. The shape of the arterial flow waveform can be calculated from the pressure signal and the two time constants of the windkessel model $\left(Z C_{w}\right.$ and $\left.R_{p} C_{w}\right)$. The value for $Z C_{w}$ has to be known at startup and the value for $R_{p} C_{w}$ is iterated with each newly detected pressure beat, assuming that the pulsatile volume stored inside the windkessel compliance remains constant over a 


\begin{tabular}{lcc}
\hline & Arterial & Pulmonary \\
\hline Mean pressure (mmHg) & $30-250$ & $5-70$ \\
Systolic pressure (mmHg) & $40-270$ & $5-95$ \\
Diastolic pressure (mmHg) & $20-200$ & $5-60$ \\
Period time (ms) & $100-3000$ & $100-3000$ \\
Ejection time (ms) & $50-2000$ & $50-2000$ \\
Ejection/Period time & $0.1-1$ & $0.1-1$ \\
\hline
\end{tabular}

Table 5.1: Criteria used to determine whether a pair of arterial and pulmonary pressure beats was acceptable. A pair of pressure beats was used in further analysis when all its values were inside the ranges shown.

longer period of time (equation 4.4, page 46). For the arterial system $Z C_{w}$ was taken at $0.12 \mathrm{~s}$ and $R_{p} C_{w}(t=0)$ at $1 \mathrm{~s}$ (chapter 4$)$. For the pulmonary pressure signal the dicrotic notch was detected in a similar fashion. Windkessel model parameters were derived empirically by visually inspecting the resulting flow curves for a range of windkessel model parameters; for all patients $Z C_{w}$ was chosen as $0.04 \mathrm{~s}$ and $R_{p} C_{w}(t=0)$ as $1 \mathrm{~s}$.

\subsubsection{Validation of pressure signals}

As a first validity test, we verified whether a pair of arterial and pulmonary pressure beats could be accepted for further analysis. A pair of pressure beats was acceptable when all of its descriptive values were inside an acceptable range (table 5.1). To test the validity of each acceptable pair of pressure beats, the beat-to-beat fluctuations in the duration of left ventricular ejection, $T_{a r t}$, the duration of right ventricular ejection, $T_{p u l}$ and the difference between the durations of left and right ventricular ejection, $\Delta T=T_{a r t}-T_{p u l}$ were analysed. For each pair of pressure beats three validation features were derived; (1) the absolute difference between $T_{\text {art }}$ and its average value, (2) the absolute difference between $T_{p u l}$ and its average value and (3) the absolute value of $\Delta T$. A pair of pressure beats was rejected if at least one of its validation feature values was too large. The maximum allowed values of a validation feature will be referred to as the validation boundaries.

\subsection{Evaluation of performance}

Recordings were made for 14 patients with irregular heart rates who were catheterized prior to cardiac surgery. The arterial pressure signal was measured using a radial artery cannula (20-gauge). The pulmonary artery pres- 


\begin{tabular}{lcccccccc}
\hline ID & MAP & MPAP & $\#$ & $\% 1$ & $T_{a r l}$ & $T_{p u l}$ & $\Delta T$ & $\%_{2}$ \\
\hline 1 & 91.6 & 33.1 & 143 & 99.3 & $314(29)$ & $319(36)$ & $-6(33)$ & 90 \\
2 & 69.4 & 23.4 & 122 & 100.0 & $342(43)$ & $334(54)$ & $8(67)$ & 55 \\
3 & 67.8 & 19.4 & 79 & 100.0 & $357(23)$ & $270(24)$ & $87(90)$ & 73 \\
4 & 75.6 & 23.4 & 109 & 99.1 & $326(22)$ & $259(20)$ & $67(71)$ & 97 \\
5 & 79.8 & 31.6 & 139 & 100.0 & $289(14)$ & $264(35)$ & $25(33)$ & 96 \\
6 & 75.9 & 20.6 & 116 & 99.1 & $342(32)$ & $310(31)$ & $32(44)$ & 89 \\
7 & 63.1 & 40.5 & 138 & 96.4 & $314(20)$ & $321(41)$ & $-7(38)$ & 86 \\
8 & 82.5 & 57.4 & 180 & 98.9 & $233(22)$ & $272(46)$ & $-39(43)$ & 90 \\
9 & 87.8 & 22.1 & 70 & 95.7 & $343(17)$ & $330(28)$ & $14(25)$ & 88 \\
10 & 71.4 & 35.6 & 79 & 100.0 & $324(17)$ & $361(27)$ & $-37(42)$ & 89 \\
11 & 71.9 & 32.8 & 156 & 90.4 & $259(29)$ & $229(28)$ & $31(36)$ & 87 \\
12 & 85.2 & 34.2 & 114 & 99.1 & $271(14)$ & $383(100)$ & $-112(113)$ & 59 \\
13 & 90.9 & 12.2 & 113 & 99.1 & $279(17)$ & $305(25)$ & $-26(30)$ & 97 \\
14 & 70.3 & 20.6 & 137 & 98.5 & $275(13)$ & $228(35)$ & $47(62)$ & 90 \\
\hline \multicolumn{7}{l}{ MAP and MPAP are in mmHg and $T_{a r l}, T_{p u l}$ and $\Delta T$ in ms. } &
\end{tabular}

Table 5.2: Results of the signal validation routine for pressure beats at an irregular rate. ID, patient identification number. MAP, mean arterial pressure. MPAP, mean pulmonary artery pressure. \#, number of beats. $\%_{1}$, percentage of acceptable pairs of pressure beats (table 5.1). $T_{\text {art }}$, duration of left ventricular ejection. $T_{p u l}$, duration of right ventricular ejection. $\Delta T$, difference between durations of left and right ventricular ejection. Values between brackets indicate the average values of the derived validation features, i.e. the absolute deviation from the mean within each patient $\left(T_{a r l}\right.$ and $\left.T_{p u l}\right)$ or from zero $(\Delta T) . \%_{2}$, percentages of valid pairs of pressure periods when the validation boundaries were taken at $n=2.5$ times the average validation feature values as found in all patient (figure 5.4).

sure was measured from the distal tip of a $7 \mathrm{~F}$ pulmonary artery catheter, which was introduced via the right jugular vein. Pressure signals were taken from the operating-room monitoring system (M1168A component monitoring system, Hewlett Packard), which had a built-in low-pass filter with its cutoff frequency at 13 Hertz. Pressure signals were digitized by an analogto-digital convertor (DAS1600, Keithley Metrabyte) with an accuracy of 12 bits for $500 \mathrm{mmHg}$ and a sampling rate of $100 \mathrm{Hertz}$. Raw pressure signals were stored on the hard disk of an IBM-AT compatible personal computer every minute for a duration of 6 seconds. A total of 20 consecutive 6 second blocks were analysed for each patient. All pressure curves were free of measurement artefacts. Data were analysed offine. Time derivatives were approximated using a 5-point data fit on a second-order polynomial.

The optimum value for $t_{d}$ was determined for each patient by finding the maximal value of $\bar{F}_{\text {corr }}$ (figure 5.2). The optimum value for $t_{d}$ ranged from -30 to $120 \mathrm{~ms}$ (average $27.5 \mathrm{~ms}$ ). When the optimum value of $t_{d}$ for 

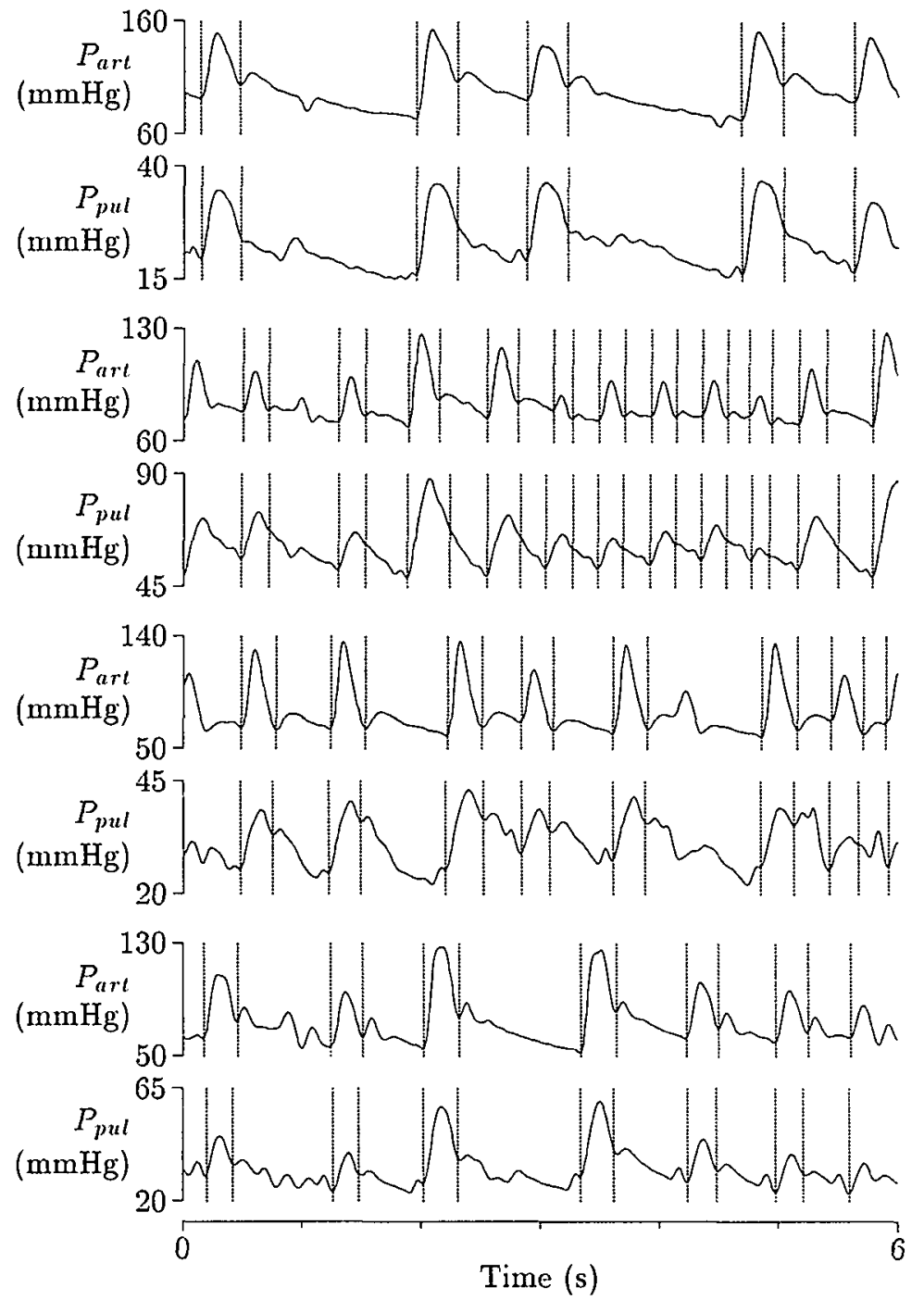

Figure 5.3: Illustrative examples of detection results in 4 patients. Shown are the radial, $P_{a r t}$, and pulmonary pressure signals, $P_{p u l}$, and the markers placed by the detection algorithm at start and end of left and right ventricular ejection. 


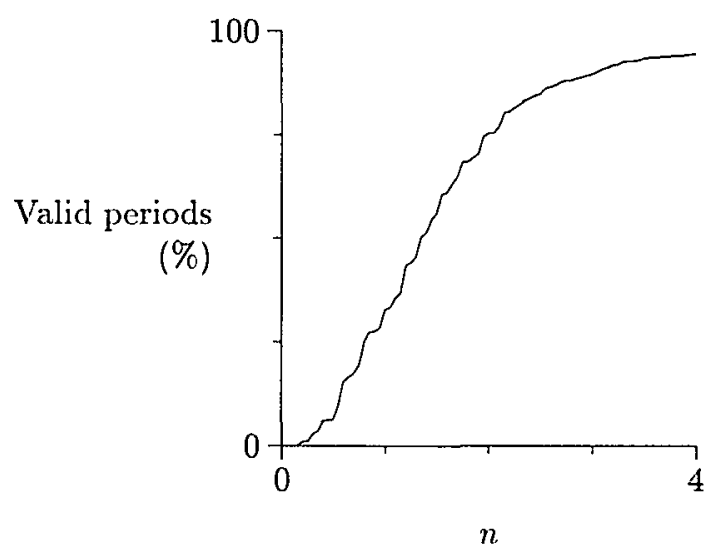

Figure 5.4: The percentage of valid pressure beats when the validation boundaries were taken at $n$ times the average values of the validation features as found in all 14 patients. The multiplier $n$ ranged from 0 to 4 in steps of 0.05 . Results for $n=2.5$ are given for each patient in table 5.2

a patient was used, a total of 1695 pressure beats were detected by the algorithm, of which $98 \%$ were acceptable (according to the criteria listed in table 5.1). When the average value of $t_{d}=30 \mathrm{~ms}$ ( 3 sampling intervals) was used, a total of 1646 pressure beats were detected by the algorithm, of which also $98 \%$ were acceptable. For further analysis $t_{d}$ was chosen at the optimal value for a given patient.

In 12 of the 14 patients a detection level for $F_{l h}$ as $15 \cdot 10^{3} \mathrm{mmHg}^{2}$ $\mathrm{s}^{-2}$ was used. In one patient the detection level had to be lowered to $5 \cdot 10^{3} \mathrm{mmHg}^{2} \mathrm{~s}^{-2}$, to prevent systolic upstrokes from being missed. In another patient the detection level had to be increased to $25 \cdot 10^{3} \mathrm{mmHg}^{2}$ $\mathrm{s}^{-2}$ to prevent the algorithm from falsely labelling increases in pressure at end-ejection as systolic upstrokes. Relevant data of all subjects are given in table 5.2.

All markers placed by the algorithm were visually inspected. In all 1659 pairs of pressure beats considered, pressure disturbances in 17 pairs of pressure beats $(1.0 \%)$ were incorrectly labelled as systolic upstrokes (false positive). A total of $59(3.5 \%)$ pairs of pressure beats were not identified by the detection algorithm (false negative), because either $F_{\text {corr }}$ did not cross the selected detection level $(1.4 \%)$ or an upstroke pressure was only visible in one of both pressure signals $(2.1 \%)$. The dicrotic notch was incorrectly determined in eight $(0.5 \%)$ and $18(1.1 \%)$ of the pressure 
beats for the arterial and pulmonary artery pressure signals respectively. It should be emphasized that visual interpretation of pulmonary pressure signals is difficult and therefore ambiguous (figure 5.3).

No significant trends in time could be detected in $T_{a r t}, T_{p u l}$ and $\Delta T$ over the $20 \mathrm{~min}$ intervals. We therefore analysed the beat-to-beat fluctuations in $T_{a r t}, T_{p u l}$ and $\Delta T$ by comparing them with the 20 -minute averaged values for each patient. The average values of the validation features derived from $T_{\text {art }}, T_{p u l}$ and $\Delta T$ were $22.3,37.8$ and 51.9 ms respectively. Figure 5.4 shows the percentages of valid periods when the validation boundaries were chosen at $n$ times these average values. Generally, too many pairs of pressure beats are incorrectly judged as valid for too large values of $n$, whereas too few beats are judged as valid for too small values of $n$. From figure 5.4 we chose the final validation boundaries for $n=2.5$. For $n=2.5$, between 55 and $97 \%$ (average $85 \%$ ) of the beats were judged as valid by the algorithm (table 5.2).

\subsection{Discussion}

At irregular heart rates the commonly used validation features, such as systolic and diastolic pressure and timing values, cannot be used owing to the large beat-to-beat variations. Results from the present study indicated that the durations of left and right ventricular ejection are almost equal and remain fairly constant over time even at irregular heart rates (table 5.2). The beat-to-beat fluctuations in $T_{a r t}, T_{p u l}$ and $\Delta T$ were small enough for them to be used for the validation of blood-pressure signals at an irregular rate. When the validation boundaries were taken at $n=2.5$ times the average deviations found in all patients, $85 \%$ of the pressure beats were judged as valid, though in two patients fewer than $60 \%$ of the pressure beats were judged as valid by the validation algorithm. From the results of chapter 3 , where on average $77 \%$ of the pressure beats were found to be valid, it follows that this will provide a sufficient percentage of valid 5 -second intervals for a closed-loop system. The disadvantage of the proposed validation technique is that it requires a double pressure signal. Furthermore, it was assumed that the durations of left and right ventricular ejection are nearly equal and constant over time. This proved to be a reasonable assumption in our patient group, but it might not hold for all patients.

As an alternative validation feature, spectral components (Kuo and Chan 1993) determined online may be considered. Aperiodic pressure signals do however not have distinct spectral components and consequently 
a large time window with a large time lag is needed to estimate spectral components reliably. Windkessel model parameter values determined online (Ocasio et al. 1993) might also be considered as validation features. However, this method does not eliminate the need to identify start and finish of ejection in each pressure beat. Moreover, for the arterial system the physiological relevance of windkessel model parameters as derived from the peripheral pressure pulse is questionable, while for the pulmonary system the peripheral resistance strongly depends on pulmonary blood flow. Neural networks have also been proposed to validate pressure signals (Pike and Mustard 1992; Odaka et al. 1994; Prentza and Wesseling 1995). They do however require a long learning time and still need a set of characteristic features to describe each pressure beat.

Detection of systolic upstrokes was facilitated by correlating the derivatives of $P_{a r l}$ and $P_{p u l}$. This was an important part of the signal validation, because the correlation factor, $F_{\text {corr }}$, will not cross the detection level, $F_{t h}$, when arterial and pulmonary systolic upstroke pressures are not synchronized. Detection of systolic upstrokes fails when one ventricle does not eject blood and thus no upstroke pressure is present in the associated pressure signal. Fortunately, this occurred only in $2.1 \%$ of the pressure beats.

The correlation technique successfully detected the systolic upstrokes in all patients, although in two patients a different detection level had to be chosen manually. We therefore investigated the possibility of computing the optimum detection level automatically for a given patient. From individual plots of $F_{\text {corr }}$ an optimum detection level, $F_{t h}$, was determined visually for each patient. Using linear regression, $F_{l h}$ could be predicted from $\bar{F}_{c o r r}$ of equation 5.2 as

$$
F_{l h}=-4.7 \cdot 10^{3}+2.7 \bar{F}_{\text {corr }}
$$

with a correlation coefficient of $r^{2}=0.91$. This suggests that the optimum value of $F_{t h}$ may be determined automatically using equation 5.3. Normalizing $F_{\text {corr }}$ by dividing it by the energy content of $\dot{P}_{a r t}$ and $\dot{P}_{p u l}$ may also be considered.

The correction for the time shift, $t_{d}$, between arterial and pulmonary pressure signals for each patient ensured that systolic upstrokes coincided and $F_{\text {corr }}$ crossed the detection threshold. When a fixed time shift. $\left(t_{d}=\right.$ $30 \mathrm{~ms}$ ) was used for all patients, 49 out of 1695 pressure beats $(2.9 \%)$ were not detected by the algorithm, suggesting that use of a fixed value for $t_{d}$ does not diminish performance significantly.

The windkessel model approach (chapter 4) successfully detected the dicrotic notch in most pressure beats, for both the arterial $(99.5 \%)$ and 
the pulmonary (98.9\%) pressure signal. It should however be stressed that even visual interpretation of the pulmonary artery pressure signal for some patients is difficult (figure 5.3). The detection of the start of ejection from the pulmonary pressure signal may greatly be improved by correcting for the characteristic dip that is observed in some patients at end diastole. Interpolation or filtering techniques may prove useful, but first further study is needed to determine the origin of the dip.

In the present study we demonstrated the feasibility of a detection and validation algorithm for arterial and pulmonary blood-pressure signals at an irregular rate. The validation algorithm needs to be further improved to determine the start of the right ventricular ejection more accurately from the pulmonary artery pressure signal. Furthermore, it should be investigated whether the detection threshold, $F_{l h}$, can be determined automatically. Future research should further evaluate the algorithm's performance on pressure recordings that also include distorted (invalid) data such as flushes of the fluid-filled line or electrical interference from coagulation equipment. 
Chapter 5 


\section{Chapter 6}

\section{The feasibility of adaptive control of blood pressure during cardiac surgery}

S. A. A. P. Hoeksel

J. A. Blom

J. R. C. Jansen

A. P. G. Hoeks

J. J. Schreuder 


\section{Abstract}

Objective. To estimate the response of patients to vasoactive drugs from closed-loop controlled blood-pressure data and to elucidate whether parameter estimates are reliable enough to allow for adaptive control.

Material and method. Mean systemic arterial pressure was closed-loop controlled using either sodium nitroprusside ( 50 patients) or nitroglycerin ( 24 patients). Mean pulmonary artery pressure was closed-loop controlled using nitroglycerin (18 patients). The single-input single-output closed-loop systems were adaptive and used rule-based logic to classify the patient sensitivity and the inversely related control gain in one of five sensitivity categories online. The patient model parameters were also estimated offline by a least squares parameter identification routine. Errors were analysed to study the accuracy of the parameter estimates.

Results. Only an indication of the range of values could be given for the parameters identified offline. Variances were too large to give reliable estimates for a given patient. The patient sensitivity that was estimated online by the blood-pressure controller was generally higher than or equal to the patient sensitivity estimated offline.

Conclusions. Closed-loop controlled blood-pressure data does not provide reliable estimates of patient model parameters for a given patient. This suggests that a strict implementation of an adaptive control law might not be feasible during cardiac surgery. Implemented expert knowledge is needed to guide the estimation process and to arrive at realistic estimates.

Keywords adaptive control $\bullet$ blood pressure $\bullet$ drug response $\bullet$ pharmacodynamic modelling 


\subsection{Introduction}

Several articles on the subject of closed-loop blood-pressure control published during the past decade (Linkens and Hacisalihzade 1990; O'Hara et al. 1992; Isaka and Sebald 1993; Jastremski et al. 1995) illustrate the continuing interest in the subject. The design of a closed-loop bloodpressure controller requires a mathematical model to predict the patient's response to changes in drug infusion rate. For most drugs the average haemodynamic response is known, but haemodynamic response varies strongly between patients and little is known of the response for a given patient. To guarantee an acceptable control performance, a control strategy is needed that identifies the patient model parameters during a control session and aclapts the control parameters accordingly (Isaka and Sebald 1993; Åström and Wittenmark 1995). Various adaptive blood-pressure controllers have been described for single-input single-output (Arnsparger et al. 1983; Meline et al. 1985; Stern et al. 1985; Millard et al. 1987; PrysRoberts and Millard 1990; Kwok et al. 1995; Maitelli and Yoneyama 1997) and multiple-input multiple-output (McInnis and Deng 1985; Voss et al. 1987; Yu et al. 1992) systems. These adaptive controllers rely heavily on the accuracy of the patient model estimated online. The high background noise in a clinical setting might however prevent a continuously reliable estimation of the patient model, resulting in a potentially unstable controller design. During cardiac surgery especially, a strict treatment of an adaptive control law might fail, because here only a short learning time is available and large pressure fluctuations independent of changes in drug infusion rate alone occur. Furthermore, identifiability and controllability are contradictory; a satisfactory control might not yield accurate parameter estimates and vice versa.

We controlled mean arterial pressure (MAP) and mean pulmonary artery pressure (MPAP) utilizing a closed-loop procedure in patients undergoing cardiac surgery, using a single vasodilator drug. Systemic hypertension was controlled using either sodium nitroprusside (SNP) or nitroglycerin (NTG). Pulmonary artery hypertension was controlled using NTG. The single-drug blood-pressure controllers were adaptive and used rule-based reasoning to classify the patient sensitivity online into one of five sensitivity categories. The results and the design of these single-drug blood-pressure controllers have been clescribed in chapters 7,8 and 9. The patient model parameter values were estimated offline by a least squares parameter identification routine. Error analyses were carried out to investigate the accuracy of the parameter estimates. The additional knowledge of the range of pa- 

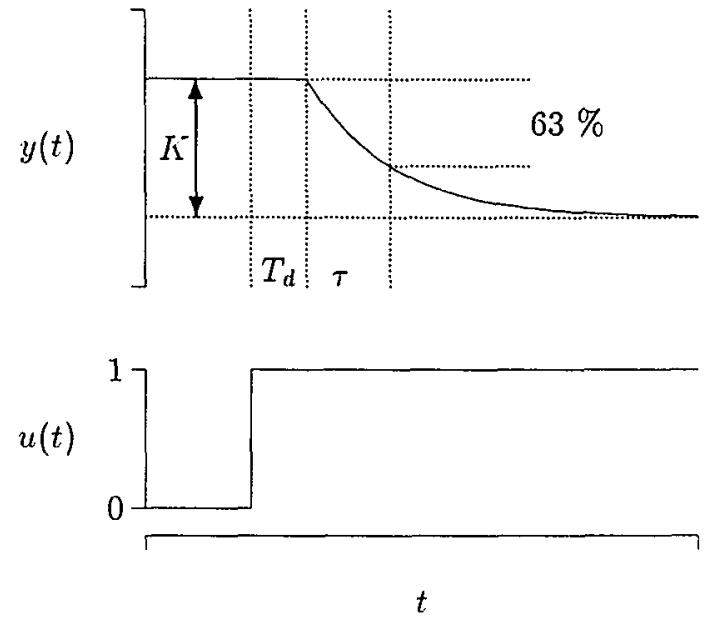

Figure 6.1: This figure shows the typical change in pressure, $y(t)$, to a unit stepwise change in drug infusion rate, $u(t)$. The delay time, $T_{d}$, models the time till the onset of drug action. The time constant $\tau$ describes the exponential decay of $y(t)$. The sensitivity, $K$, is the pressure change when $t$ approaches infinity.

tient model parameters might be helpful in designing more stable and perhaps faster blood-pressure controllers. The error analysis might be helpful in elucidating the extent to which a strict treatment of an adaptive control law is feasible during cardiac surgery.

\subsection{Material and method}

\subsubsection{Modelling patient response}

In an open-loop setting Sheppard (1980) used cross-correlation analysis and pseudo-random binary changes in SNP infusion rate to identify the dynamic response of MAP. The MAP response could be modelled by a first-order transfer function

$$
\begin{aligned}
H(s) & =\frac{Y(s)}{U(s)} \\
& =\left(1+\alpha \mathrm{e}^{-T_{c} s}\right) \mathrm{e}^{-T_{d} s} \frac{K}{1+s \tau}
\end{aligned}
$$

where $Y(s)$ and $U(s)$ are the Laplace transforms of respectively the induced pressure change, $y(t)$ and the drug infusion rate, $u(t)$ (figure 6.1). 
$T_{d}$ represents the initial transport lag from the injector site to the target compartment and is approximately equal to one circulation time. $K$ is the patient sensitivity and relates the drug concentration in the target compartment to a given decrease in blood pressure. The drug elimination from the target compartment is described by a first-order process with time constant $\tau$. The recirculation factor, $\alpha$, is the ratio of drug quantity that recirculates within $T_{c}$ seconds. In many studies, including the present one, the effect of recirculation is neglected.

For several reasons, we chose a first-order model (equation 6.1) to describe the patient response to changes in drug infusion rate. First, a firstorder model has been used previously in closed-loop blood-pressure controllers to model the patient response to various drugs, including SNP (Sheppard 1980), NTG (Colvin and Kenny 1989), halothane (Smith and Schwede 1972), isoflurane (Prys-Roberts and Millard 1990) and phenylephrine (Millard et al. 1987). Second, a first-order model describes the first terms of a power-series development of a linearization of the true patient response. Third, the step response of a first-order model (figure 6.1) provides sufficient parameters to tune a proportional-integral regulator ( $\AA$ ström and Hågglund 1995) such as was used in our controllers (chapters 7,8 and 9).

Since digital signal processing was to be used, we first needed to derive a time discrete representation of equation 6.1. The impulse response of equation 6.1 , for $\alpha$ set to zero, is given by

$$
h_{c}(t)= \begin{cases}0 & t<T_{d} \\ K \tau^{-1} \mathrm{e}^{-\frac{t-T_{d}}{\tau}} & t \geq T_{d}\end{cases}
$$

The infusion rate $u(t)$ is changed every $T_{s}$ seconds and can thus be described by a zero-order hold process

$$
u(t)=u\left(n T_{s}\right) \quad n T_{s} \leq t<(n+1) T_{s}
$$

The output $y(t)$ that is observed at the sampling instances $n T_{s}$ is now given by

$$
\begin{aligned}
y\left(n T_{s}\right) & =\int_{-\infty}^{\infty} h_{c}(t) u\left(n T_{s}-t\right) d t \\
& =\sum_{k=-\infty}^{\infty} \int_{(k-1) T_{s}}^{k T_{s}} h_{c}(t) u\left(n T_{s}-t\right) d t \\
& =\sum_{k=-\infty}^{\infty} u\left((n-k) T_{s}\right) h(k)
\end{aligned}
$$


where

$$
\begin{aligned}
h(n) & =\int_{(n-1) T_{s}}^{n T_{s}} h_{c}(t) d t \\
& = \begin{cases}0 & n<n_{d}+1 \\
-K^{-}\left(1-\mathrm{e}^{T_{s} / \tau}\right) \mathrm{e}^{-\frac{\left(n-n_{d}\right) T_{s}}{\tau}} & n \geq n_{d}+1\end{cases}
\end{aligned}
$$

and $n_{d}$ equals $T_{d} / T_{s}$ rounded off to the nearest integer. The Z-transform of the discrete impulse response 6.2 is given by

$$
H(z)=K\left(1-\mathrm{e}^{-T_{s} / \tau}\right) \frac{z^{-n_{d}}}{z-\mathrm{e}^{-T_{s} / \tau}}
$$

Introducing the short-hand notations $y\left(n T_{s}\right)=y(n)$ and $u\left(n T_{s}\right)=u(n)$, the input-output relation of the patient response in cliscrete time follows from the Z-transform of equation 6.3.

$$
y(n)+a_{1} y(n-1)=b_{0} u\left(n-n_{d}-1\right)+\varepsilon(n)
$$

where

$$
\begin{aligned}
& a_{1}=-\mathrm{e}^{-T_{s} / \tau} \\
& b_{0}=K\left(1-\mathrm{e}^{-T_{s} / \tau}\right)
\end{aligned}
$$

and $\varepsilon(n)$ is an equation error. Note that no approximations are used in deriving equations $6.4,6.5$ and 6.6 as long as $T_{d}$ is an exact multiple of $T_{s}$. Since $T_{d}$ is unknown and variable, this cannot be guaranteed in practice.

\subsubsection{Offline identification of patient model}

It can be shown that model 6.4 is identifiable in a closed-loop setting for values of $n_{d}$ greater than zero (Gessing and Blachuia 1996). An equation error approach was used, because filtering of the noise sequence $\varepsilon(n)$, as for example with output error models or correlation analysis, would bias results owing to the feedback in the data. The equation error approach uses the difference equation 6.4 evaluated at the times $1 \ldots N$. Introducing the matrices

$$
Y=\left[\begin{array}{l}
y\left(n_{d}+2\right) \\
\vdots \\
y(N)
\end{array}\right]
$$




$$
\begin{aligned}
\Omega & =\left[\begin{array}{ll}
-y\left(n_{d}+1\right) & u(1) \\
\vdots & \vdots \\
-y(N-1) & u\left(N-n_{d}-1\right)
\end{array}\right] \\
\theta & =\left[\begin{array}{l}
a_{1} \\
b_{0}
\end{array}\right]
\end{aligned}
$$

the one-step ahead prediction of the output vector $Y$ is given by

$$
\hat{Y}=\Omega \theta
$$

The parameter vector, $\theta$, that minimizes the least squares error criterium

$$
J(\theta)=\sum_{k=n_{d}+2}^{N} \varepsilon(k)^{2}=(Y-\hat{Y})^{T}(Y-\hat{Y})
$$

is given by

$$
\hat{\theta}=\left(\Omega^{T} \Omega\right)^{-1} \Omega^{T} Y=\Omega^{+} Y
$$

where $\Omega^{+}$is the pseudo-inverse of $\Omega$. Offsets in the model were corrected for by subtracting the sample means from the signals $y(n)$ and $u(n)$.

It can be shown that $\hat{\theta}$ is a minimum variance estimator when the noise sequence $\varepsilon(n)$ is white (Hsia 1977; Ljung 1987). Correlation analysis can be used to test the whiteness of $\varepsilon(n)$ a posteriori. The whiteness of $\varepsilon(n)$ can be verified by calculating the ratio of the number of positive zero-crossings of the auto-correlation of $\hat{\varepsilon}(n)$ and $N-\left(n_{d}+2\right)$ (Hsia 1977), which statistically should be equal to 0.5 .

The estimated parameter values of $\hbar^{-}$and $\tau$ can be found from $\hat{a}_{1}$ and $\hat{b}_{0}$ (equations 6.5 and 6.6 )

$$
\begin{aligned}
\hat{\tau} & =\frac{-T_{s}}{\ln \left(-\hat{a}_{1}\right)} \\
\hat{K}^{-} & =\frac{\hat{b}_{0}}{1+\hat{a}_{1}}
\end{aligned}
$$

The sampling time, $T_{s}$, of the closed-loop controllers was chosen as $5 \mathrm{sec}$ onds. When estimating $K$ and $\tau$ the delay $n_{d}$ was assumed to be nine sampling intervals (i.e 45 seconds) in all patients. An estimate of $n_{d}$ for a given patient was obtained by finding the value of $n_{d}$ that provided the best fit in a least squares sense when $n_{d}$ was varied from $1 \ldots 19$ sampling intervals (Hsia 1977). Estimates of $n_{d}$ that were equal to the extreme values were considered unreliable and were therefore rejected. 


\begin{tabular}{lccc}
\hline & \multicolumn{2}{c}{ MAP } & MPAP \\
\cline { 2 - 3 } & NTG & SNP & NTG \\
\hline$T_{\boldsymbol{d}}(\mathrm{s})$ & 45 & 45 & 45 \\
$\tau(\mathrm{s})$ & 175 & 60 & 175 \\
$K^{-}\left(\mathrm{mmHg} \mu \mathrm{g}^{-1} \mathrm{~kg} \mathrm{~min}\right)$ & $-6.5:-68$ & $-6.3:-100$ & $-3.2:-34$ \\
\hline
\end{tabular}

Table 6.1: Model parameter values that were used for the tuning of the singleinput single-output blood-pressure controllers. For the sensitivity, $K$, the range is given. $\tau$, time constant. $T_{d}$, delay time. MAP, mean arterial pressure. MPAP, mean pulmonary artery pressure. SNP, sodium nitroprusside. NTG, nitroglycerin.

When the residuals $\varepsilon(n)$ are identically distributed and independent with zero mean and variance $\sigma^{2}$, the covariance matrix of the estimation error $\hat{\theta}-\theta$ can for large values of $N$ be approximated by

$$
\Psi=\sigma^{2}\left(\Omega^{T} \Omega\right)^{-1}
$$

where an estimate of $\sigma^{2}$ can be obtained from the sample variance of the noise sequence $\hat{\varepsilon}(n)$. The variances of $\hat{\tau}$ and $\hat{K}\left(\hat{\sigma}_{\tau}^{2}, \hat{\sigma}_{K}^{2}\right)$ were calculated from $\Psi$ and the first-order terms of a Taylor series expansion of equations 6.9 and 6.10 around $\hat{\tau}$ and $\hat{K}$. The coefficient of variation, $\hat{\sigma}_{K} / \hat{K}^{*}$, was used to evaluate the variance in $K$.

\subsubsection{Online classification of patient sensitivity}

The single-drug blood-pressure controllers comprised a proportional-integral regulator and a supervisory safety shell that adapted or overruled the proportional-integral regulator when necessary (chapter 7 ). When properly tuned the proportional-integral regulator could adequately deal with a wide range of variations in the time constant $\tau$. The range in $K^{\circ}$, however, was too large to be handled by a fixed proportional-integral regulator. Simulation results revealed that control became unstable when $K$ was underestimated by factor 2 , whereas control became unacceptably slow when $K$ was overestimated by factor 2. For this reason the range of $K$ (table 6.1) was subdivided into five sensitivity categories. Each sensitivity category had a control gain associated with it. Control was stable provided that the patient's sensitivity, $K$, was correctly classified. Values for $\tau$ and the ranges of $K^{-}$(table 6.1) were taken from previously published studies (Fahmy 1978; Sheppard 1980; Blom 1991). In was classified online using rule-based logic, by comparing the expected performance of the proportional-integral regulator with the observed control performance. At start-up the patient was 


\begin{tabular}{lccc}
\hline & \multicolumn{2}{c}{ MAP } & \multicolumn{1}{c}{ MPAP } \\
\cline { 2 - 3 } & \multicolumn{1}{c}{ NTG } & SNP & NTG \\
\hline$\hat{T}_{d}(\mathrm{~s})$ & $42 \pm 26(11)$ & $51 \pm 21(16)$ & $52 \pm 31(10)$ \\
$\hat{\tau}(\mathrm{s})$ & $226 \pm 305$ & $163 \pm 190$ & $188 \pm 366$ \\
$\hat{K}(\mathrm{mmHg}$ & $-5.7 \pm 10.7$ & $-10.5 \pm 12.1$ & $-6.7 \pm 11.8$ \\
\multicolumn{1}{c}{$\mu \mathrm{g}^{-1} \mathrm{~kg}$ min $)$} & & & \\
\hline
\end{tabular}

Table 6.2: Results of offline parameter estimates. For the estimated dead time, $\hat{T}_{d}$, the number between brackets indicates the number of valid estimates used in calculating the mean and SD. $\hat{\tau}$, estimated time constant. $\hat{K}$, estimated patient sensitivity. MAP, mean arterial pressure. MPAP, mean pulmonary artery pressure. NTG, nitroglycerin. SNP, sodium-nitroprusside.

assumed to be in the second sensitivity category. The classification was decreased when control was unstable and increased when the speed of control was too slow. A set of expert rules guided the automatic classification of the patient sensitivity at all times.

\subsubsection{Patients and anaesthesia}

The blood-pressure controllers were used in 92 patients requiring vasodilator drug treatment during cardiac surgery, before and after cardiopulmonary bypass. MAP was controlled in 74 patients using either SNP $(n=50)$ or NTG $(n=24)$. MPAP was controlled in 18 patients using NTG. The study was approved by our hospital's medical ethics committee. Informed consent was obtained from patients on the evening before surgery. Standard continuous intravenous anaesthesia with opioids was used for all patients. The SNP (Nipride, Roche, Mijdrecht, the Netherlands) and NTG (Nitro Phol, Phol-Boskamp, Hohen Lockstedt, Germany) were dissolved to a concentration of $200 \mu \mathrm{g} \mathrm{ml}^{-1}$. The infusion rates of SNP and NTG were limited to respectively 3 and $5 \mu \mathrm{g} \mathrm{kg}^{-1} \mathrm{~min}^{-1}$. Infusion lines were connected to the introduction sheet of the pulmonary artery catheter. A continuous infusion of $150 \mathrm{ml} \mathrm{hr} \mathrm{hr}^{-1}$ sodium chloride was added to minimize dead time at start-up.

Offline parameter estimates were made independently from the data before and after cardiopulmonary bypass. Only data where the average infusion rate was more than $0.25 \mu \mathrm{g} \mathrm{kg}^{-1} \mathrm{~min}^{-1}$ and non-zero for more than $75 \%$ of the time were included. Results were averaged when estimates were available both before and after cardiopulmonary bypass. 


\subsection{Results}

The durations of the measurement period before and after cardiopulmonary bypass were respectively $47.4 \pm 21.9$ (mean $\pm \mathrm{SD}$ ) and $47.3 \pm 20.0 \mathrm{~min}$ utes. Offline estimates of $\tau, K^{-}$and $T_{d}$ for the MAP-SNP, MAP-NTG and MPAP-NTG controllers are given in table 6.2. The ratios of the number of positive zero-crossings of the auto correlation of $\hat{\varepsilon}(n)$ and $N-\left(n_{d}+2\right)$ for the controllers MAP-SNP, MAP-NTG and MPAP-NTG were respectively $0.47 \pm 0.06,0.51 \pm 0.05$ and $0.53 \pm 0.06$. The offline estimated $\pi^{-}$was positive in four out of 50 , six of 24 and one of 18 cases for the MAP-SNP, MAP-NTG and MPAP-NTG controllers, respectively. Figure 6.2 shows the absolute ratio of $\hat{\sigma}_{K}$ and $\hat{K}^{-}$as a function of $\hat{K}$. Figure 6.3 shows the distribution of the sensitivity categories that were estimated online by the blood-pressure controller near the sensitivity category estimated offline.

\subsection{Discussion}

The least squares estimates obtained offline could only give a rough indication of the ranges of the patient model parameters. The values for the time constant $\tau$ (table 6.2) appeared to be higher than the initial assumptions (table 6.1) that were used in the design phase of the single-drug bloodpressure controllers. This might partly be explained by the fact that when the patient model is poorly identifiable $-\hat{a}_{1}$ will tend to unity and $\hat{b}_{0}$ will tend to zero, which according to equation 6.9 implies that values for $\tau$ will be overestimated. Moreover, $\tau$ might also be overestimated when the influence of the recirculation factor $\alpha$ (equation 6.1) cannot be neglected. The large standard deviations in the parameter values and the positive values for $\hat{K}$ in some patients, illustrated that no accurate estimations could be derived for a given patient. A reliable estimate of the delay $n_{d}$ could only be obtained in $40 \%$ of the patients. In the other cases an unrealistically low or high value was estimated for $n_{d}$.

No attempts were made to estimate the patient model parameters in a recursive fashion since narrowing of the estimation horizon would only increase the variance of the estimates. The variance of the estimates would further be increased by the start-up procedure of the recursive estimator and the need to identify the offset in the model explicitly. Furthermore, the inclusion criteria that ensured a persistently exciting input (mean infusion rate higher than $0.25 \mu \mathrm{g} \mathrm{kg}^{-1} \mathrm{~min}^{-1}$ and infusion rate non-zero for more than $75 \%$ of the time), cannot be used by a recursive estimator. From simulation results it followed that a proportional-integral regulator 

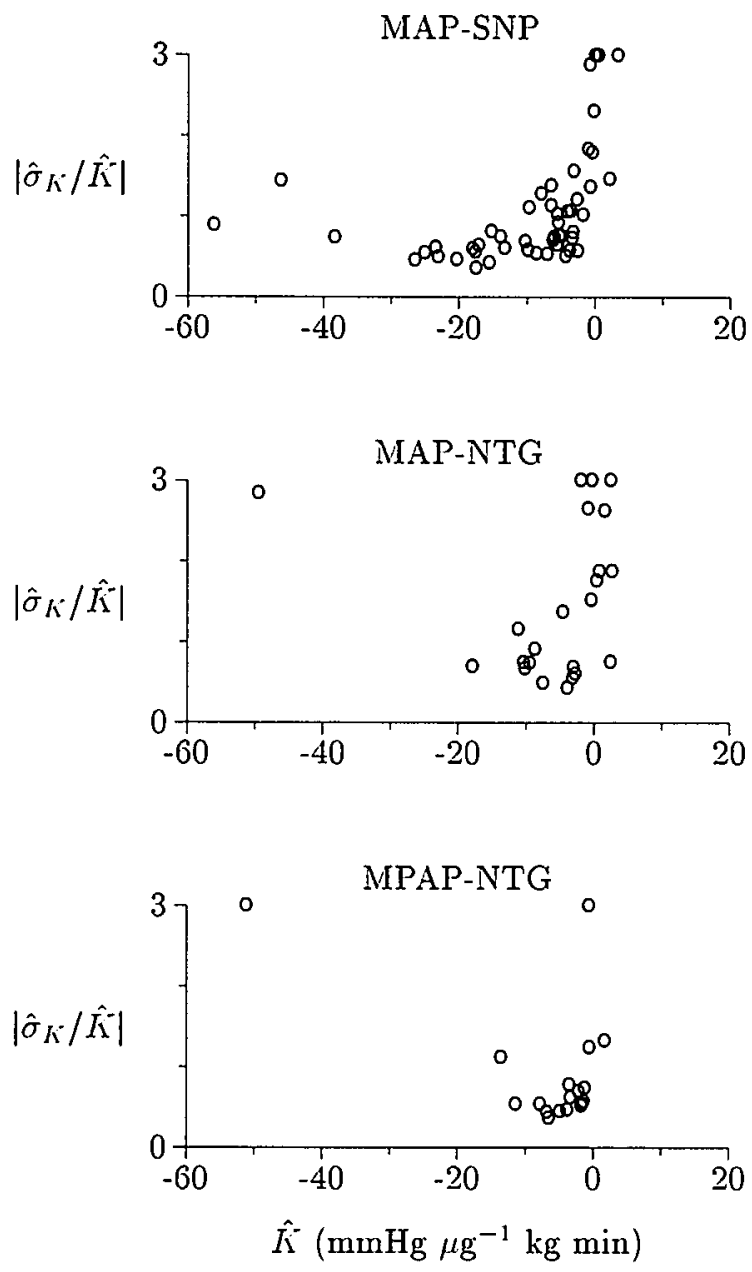

Figure 6.2: The absolute coefficient of variation, $\left|\hat{\sigma}_{K} / \hat{K}\right|$, for all controllers. The value of $\left|\hat{\sigma}_{K} / \hat{K}\right|$ indicates the accuracy of the offline estimated patient sensitivity, $\hat{K}$. For illustrative purposes, all values $\left|\hat{\sigma}_{K} / \hat{K}\right|>3$ were plotted at $\left|\hat{\sigma}_{K} / \hat{K}\right|=3$. MAP, mean arterial pressure. MPAP, mean pulmonary artery pressure. SNP, sodium-nitroprusside. NTG, nitroglycerin. 


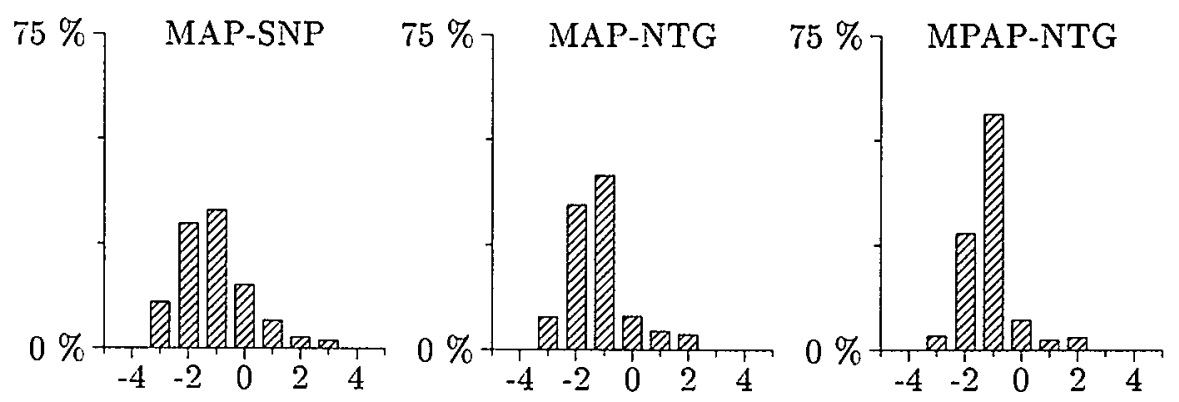

Figure 6.3: Distribution of the sensitivity categories that were estimated online by the closed-loop system around the sensitivity category estimated offline. The $\mathrm{Y}$-axis shows the percentages of time that the patient was classified in a sensitivity category. Numbers on the $\mathrm{X}$-axis indicate the deviation in number of categories from the offline estimated category. A negative value on the $\mathrm{X}$-axis indicates that the online algorithm categorized the patient as more sensitive than the offline estimation routine. MAP, mean arterial pressure. MPAP, mean pulmonary artery pressure. SNP, sodium nitroprusside. NTG, nitroglycerin.

can handle a mismatch between the true $K$ and $\hat{K}$ of factor 2, implying that the ratio $\left|\hat{\sigma}_{K} / \hat{K}\right|$ should be well below unity for all patients. When all patients with a positive value for $\hat{K}^{-}$and with $\left|\hat{\sigma}_{K} / \hat{K}^{-}\right|$larger than unity are excluded (figure 6.2), it follows that an adaptive controller using a recursive least squares estimator would result in an unacceptable control performance in at least respectively $40 \%, 58 \%$ and $28 \%$ of the patients for the MAP-SNP, MAP-NTG and MPAP-NTG controllers. Theoretically, modelling errors might be reduced when additional drug infusion rates are considered as input signals instead as system noise, but the identification of the drug cross-effects and the fact that in practice not all external input signals can be considered makes identification of such a multiple-input model even more difficult.

Model identification during cardiac surgery is difficult, because (bolus) infusions of other drugs, changes in the patient's condition and surgical manipulations may provoke changes in blood pressure that are not correlated with changes in vasodilator drug infusion rate. Other sources of error are introduced by the aliasing effects of the cyclic ventilation disturbances and heart-rate variability on the mean pressures values (TenVoorde et al. 1994a; TenVoorde et al. 1994b). Also, cyclic fluctuations in blood pressure, as are often observed in diabetic patients, might worsen estimation results. Furthermore, the direct actions of the changes in drug infusion rate are counter-acted by the patient's own pressure-regulation mechanisms 
(Guyton et al. 1972). The compensatory reflexes of the baroreceptor pressure regulatory system are for example illustrated by the blood-pressure response to an abrupt withdrawal of SNP and NTG infusions (Todd et al. 1982). The working point of the baroreflex regulatory mechanism might also be reset and an imposed target pressure might be maintained even after drug infusion is discontinued (Incalzi et al. 1989).

Another possible source of error is in the assumption that the true patient response can sufficiently be described by a first-order model. A first-order model can be viewed as a linearization around a working point and its usefulness in predicting the patient response to larger changes in drug infusion rate is questionable. Response to vasoactive drugs is known to be nonlinear and the linearization around a small working range makes it likely that the parameter values of $\tau$ and $I^{*}$ depend on pressure levels, but most likely also on other variables, such as cardiac output. The patient response might also vary over time as the condition of the patient changes. Owing to the limited information content of the data and the relatively short measurement intervals before and after cardiopulmonary bypass, no information concerning pressure and time dependencies could be inferred from the data of the present study. However, correlation analysis of $\hat{\varepsilon}(n)$ indicated that a first-order model appears to be an acceptable approximation.

Feedback not only restricts the model identification technique to an equation error approach, but also minimizes fluctuations in input and output, which conflicts with the condition of a sufficiently rich input needed for the identification process. Results from this study suggest that inputoutput data from a blood-pressure controller with control performances acceptable for the clinician do not have sufficient innovations to allow for reliable parameter estimations. This is indicated by the fact that a relatively high number of estimates of $\hat{K}$ are centred around zero (figure 6.2). With adaptive control these low values for $\hat{K}$ will result in an aggressive control, such that more disturbances are introduced and more accurate estimates of $K$ can be obtained in future. In a clinical setting however these large variations in blood pressure and drug infusion rate should be avoided and reliable parameter estimates may therefore only be obtained in animal experiments.

The gain adaptation mechanism that was used in our single-drug closedloop blood-pressure controllers assumed a fixed value for $\tau$ and used rulebased logic to classify the patient's $K$ into one of five sensitivity categories. This ensured that no unrealistic control gains were selected and that a form of common sense was applied when the patient sensitivity was being esti- 
mated. Our somewhat rough-and-ready classification of patient sensitivity in five categories provided sufficient information to adapt the proportionalintegral controller and ensured stable control for all patients. It can be seen from figure 6.3 that the sensitivity estimated online was generally equal to or higher than the offline estimations, suggesting that control gain had never been chosen too high. The fact that the $K$ estimated online was higher than the $K^{-}$estimated offline might partly be explained by the fact that a patient's $K$ was assumed to be sensitive (second category) at start-up.

In conclusion we can state that least squares identification of drug response during cardiac surgery can give some idea of the parameter values which may be encountered, but accurate identification and thus prediction of the patient response is not possible for a given patient. The feasibility of a strict mathematical implementation of an adaptive control law is therefore dubious during cardiac surgery. We successfully controlled blood pressures by using expert knowledge to guide the estimation process and to arrive at realistic estimates for the patient model parameters. 


\section{Chapter 7}

\section{Automated infusion of nitroglycerin to control arterial hypertension during cardiac surgery}

S. A. A. P. Hoeksel

J. J. Schreuder

J. A. Blom

J. G. Maessen

O. C. K. M. Penn

Intensive Care Medicine 22(7) 1996, 688-693. 


\begin{abstract}
Objective. To evaluate the feasibility of closed-loop blood pressure control during cardiac surgery.

Method. A closed-loop system regulated intraoperative hypertension by controlling the infusion rate of the vasodilator nitroglycerin. The controller comprised of a proportional-integral regulator which was monitored by a supervisory computer program. Mean arterial pressure was calculated every five seconds from measurements of the radial artery pressure signal. The regulator calculated a nitroglycerin infusion rate with each new pressure measurement. The supervisory computer program monitored the regulator's actions and adapted or overruled the regulator when required. Included were 46 patients who were awaiting cardiac surgery and who developed intraoperative hypertension. The closed-loop system was used to control hypertension before and after cardiopulmonary bypass. The use of the closed-loop system did not require a deviation from the protocol normally used during cardiac surgery. All patients received standard continuous anaesthesia with opioids.

Results. Initial automatic control was achieved in $9.4 \pm 4.1$ (mean \pm SD) minutes. The percentage of time that mean arterial pressure remained in a range around the target pressure of $\pm 10 \mathrm{mmHg}$ and $\pm 20 \mathrm{mmHg}$ was respectively 74 and $94 \%$. The mean nitroglycerin infusion rate while mean arterial pressure was within $5 \mathrm{mmHg}$ of the target pressure was $1.14 \pm 0.84$ $\mu \mathrm{g} \mathrm{kg}^{-1} \mathrm{~min}^{-1}$. The target pressure was set between 65 and $90 \mathrm{mmHg}$. There was a small group of patients (six out of 46) who did not respond to nitroglycerin and required alternative drug therapy.

Conclusions. The controller provided fast and stable control in all patients. The expert knowledge implemented through the supervisory computer program enabled the controller to respond adequately to the rapid changes in arterial pressures commonly associated with cardiac surgery. We conclude that closed-loop control of arterial pressure is feasible not only in the cardiac surgical care unit, but actually during cardiac surgery.
\end{abstract}

Keywords nitroglycerin $\bullet$ computer control • drug delivery systems • cardiac surgery $\bullet$ hypertension 


\subsection{Introduction}

Vasodilator drug treatment during and after cardiac surgery requires continuous monitoring of arterial pressure and adjustment of the infusion rate of the vasodilator. This repetitive action can, in principle, be taken over by a closed-loop system. Simple feedback of the arterial pressure signal will, however, not suffice, since patient characteristics may vary greatly and pressure changes are not always related to the infusion rate of the vasodilator.

The review by O'Hara et al. (1992) reports that several investigators have successfully introduced closed-loop arterial pressure controllers into the intensive care unit. The vasodilator used in these systems has been sodium nitroprusside (SNP), but the use of nitroglycerine (NTG) has also been reported. Chitwood et al. (1992) demonstrated that closed-loop arterial pressure control using SNP significantly improves outcome for the postoperative cardiac surgery patient. Compared with manual control, closed-loop control decreases the incidence of therapy-induced hypotension, reduces postoperative bleeding, decreases transfusion requirements and probably lowers the incidence of re-exploration for postoperative bleeding.

The introduction of closed-loop arterial pressure controllers during cardiac surgery is complicated, because the patient's condition may rapidly change and faster control of arterial pressure is needed than in the intensive care unit. We evaluated the clinical feasibility of closed-loop control of arterial pressure during cardiac surgery. For the vasodilator we chose NTG, since it is routinely used in our institution to control intraoperative hypertension during cardiac surgery (Fahmy 1978; Kaplan and Jones 1979).

\subsection{Material and method}

\subsubsection{Patients}

The feasibility of the arterial pressure controller was studied in 46 patients who were scheduled for open heart surgery and who required vasodilator drug treatment. The study was approved by our hospital's medical ethics committee and was conducted according to the principles stated in the Helsinki convention. Informed consent was obtained from patients on the evening before surgery. Data for six patients were discarded from statistical analysis because their arterial pressure did not decrease after the closedloop system had increased the NTG infusion rate. In these cases, manually 
adjusted infusion of SNP was chosen to control hypertension and the study programme was stopped. Of the remaining 40 patients, 26 were scheduled for coronary bypass surgery and 14 underwent valve replacement surgery. Most patients were male (28 of 40), aged 41 to 77 years (average 64 years). Patients' weights varied between 55 and $106 \mathrm{~kg}$ (average $76 \mathrm{~kg}$ ).

\subsubsection{Anaesthesia}

Standard continuous intravenous anaesthesia with opioids was administered to all patients. All patients received lorazepam as oral premedication two hours before surgery. Patients scheduled for coronary bypass surgery received triple medication according to a general protocol including nitrates, $\beta$-blockades and Ca-antagonists. Anaesthesia was induced with $7.5 \mu \mathrm{g} \mathrm{kg}^{-1}$ sufentanil (Janssen Pharmaceutica, Beerse, Belgium) and maintained with $3.75 \mu \mathrm{g} \mathrm{kg}^{-1} \mathrm{~h}^{-1}$ sufentanil. Muscle relaxation was achieved with $0.1 \mathrm{mg}$ $\mathrm{kg}^{-1}$ pancuronium bromide. The lungs were ventilated with an oxygen/air mixture $\left(\mathrm{F}_{\mathrm{i}} \mathrm{O}_{2}=0.50\right)$ at a rate of 12 breaths $\mathrm{min}^{-1}$ and ventilatory volume was adjusted to maintain arterial $\mathrm{CO}_{2}$ tension between 32 and $42 \mathrm{mmHg}$. Patients who underwent coronary bypass surgery received, independently of the study programme, a baseline infusion of NTG $\left(0.5 \mu \mathrm{g} \mathrm{kg}^{-1} \mathrm{~min}^{-1}\right)$ after cardiopulmonary bypass to prevent spasm of the mammary artery. Additional dopamine or dobutamine was given during the operation if the cardiac index was less than $2 \mathrm{~L} \mathrm{~min}-1 \mathrm{~m}^{-2}$. Different sedatives and anaesthetics (lorazepam, sufentanil, alfentanil or isoflurane) were added to the basic anaesthetic regime when insufficient anaesthetic levels were suspected during episodes of persistent hypertension. A $7 F$ pulmonary artery catheter was introduced prior to surgery via the right internal jugular vein. The systemic arterial pressure signal was obtained using a saline-filled radial artery catheter (20 gauge) and pressure transducers (UniFlow, Baxter).

\subsubsection{Controller}

Among patients, there is a great range in the length of time required for the pressure to return to its control value after discontinuing NTG infusion and also in the required NTG infusion rate (Fahmy 1978). This variation in patient sensitivity to NTG was too large for a fixed control scheme and necessitated an adaptive control strategy. Other design criteria for the controller were; validation of the measured pressure signal, detection of unexpected periods of hypertension or hypotension, a software-indepenclent watchdog (safety shell) and a clear indication of the controller's status, 


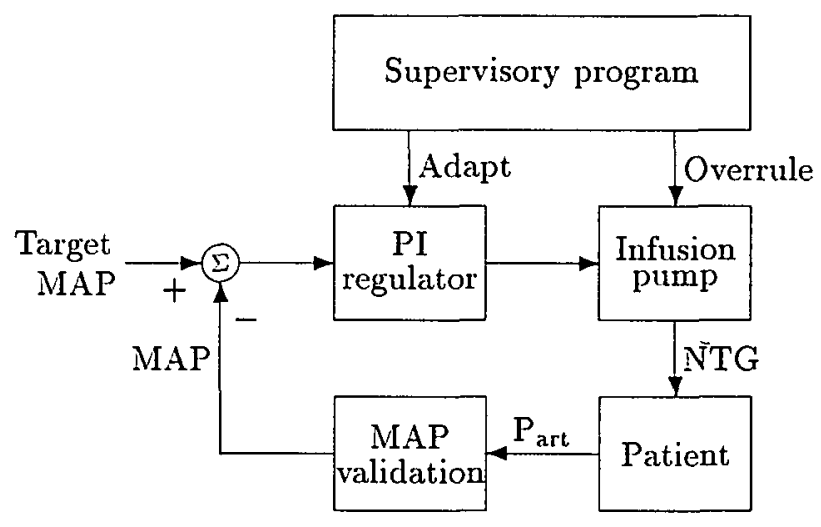

Figure 7.1: Layout of the closed-loop arterial pressure controller. MAP, mean arterial pressure. PI, proportional-integral. NTG, nitroglycerin.

including a display of trends in arterial pressures and NTG infusion rate. Most importantly, the design had to be foolproof at all times and for all patients.

Figure 7.1 represents the configuration of the arterial pressure controller. The system was based on an early prototype described by Blom (1991). The arterial pressure signal was taken from the pressure module (M1006A, Hewlett Packard) of the operating-room monitoring system (M1168A Component Monitoring System, Hewlett Packard). The signal was digitized with a 12-bit analog-to-digital convertor (DAS1600, Keithley Metrabyte) after anti-aliasing filtering at a cutoff frequency at 13 Hertz. We used the mean arterial pressure (MAP) rather than the systolic pressure as the input of the closed-loop system since its measurement is less sensitive to artefacts. The validity of each pressure period was tested by comparing its shape with the shape of the previous pressure periods. The shape of a pressure period was described by a set of characteristic features, including systolic, diastolic and mean pressure and timing values. MAP was calculated every five seconds by averaging the pressure samples of the valid pressure periods and was considered valid if at least two pressure periods were found to be valid.

A proportional-integral (PI) regulator calculated the NTG infusion rate as a linear function of the difference from the target MAP and the integral of that difference. The control gain of the PI regulator depended on the automatic classification of the patient to one of five sensitivity categories. 
The patient response to NTG was modelled as a first-order model with a time delay. The parameters of this model were expected to vary between patients and slowly with time. The PI regulator was tuned using the nominal patient model and assuming a correct classification of the patient in one of the five sensitivity categories.

A rule-based expert system was used as a supervisor and adapted or overruled the PI regulator when necessary. The supervisory computer program classified the patient in one of the five sensitivity categories and thus adapted the control gain of the PI regulator. The control gain was decreased when the MAP change during the previous 30 seconds was too fast or when an oscillation in the MAP signal had been detected, whereas the control gain was increased when the MAP change during the previous 200 seconds was too slow. At start-up the patient was assumed to be of the second sensitivity category, i.e. sensitive but not extremely sensitive. Furthermore, the supervisor detected large unexpected pressure changes by evaluating the rate of pressure change per unit of time. The PI regulator was overruled during these pressure changes, since they are usually not caused by changes in the NTG infusion rate but by pain or surgical manipulations, for example. During a sudden period of hypertension, the NTG infusion rate was maintained constant, whereas it was kept at zero during sudden periods of hypotension. Time-out mechanisms were added to ensure continuation when the pressure changes were found to be permanent after all. Moreover, the supervisory program processed user input and provided alarms in cases of invalid measurements or pump communication errors. The entire system was implemented on an IBM-AT compatible personal computer and programmed in Turbo Pascal version 7.0 (Borland International Inc., Scotts Valley, CA, USA).

The calculated NTG infusion rate was corrected for the patient's weight and transmitted to a syringe infusion pump (IVAC P4000, IVAC Corporation) or a volumetric infusion pump (IMED 929, IMED Corporation) using a RS232 serial interface. Both infusion pumps require regular communication to prevent a time-out alarm and thus provided a software-independent watchdog. An extensive software handshake protocol ensured safe communication between computer and infusion pump. The NTG infusion (Nitro Phol, Phol-Boskamp, HohenLockstedt, Germany) was prepared by dissolving the NTG in glucose to a concentration of $40 \mu \mathrm{g} \mathrm{ml}^{-1}$ (for use with the IMED 929) or $200 \mu \mathrm{g} \mathrm{ml}^{-1}$ (for use with the IVAC P4000). The NTG infusion line was connected to the side-port of the pulmonary artery catheter. A continuous infusion $\left(150 \mathrm{ml} \mathrm{hr}^{-1}\right)$ of sodium chloride $(0.9 \%)$ was added to minimize dead-time at start-up. The maximum NTG infusion rate was 
$3 \mu \mathrm{g} \mathrm{kg}^{-1} \mathrm{~min}^{-1}$ during the first 14 cases and was raised to $5 \mu \mathrm{g} \mathrm{kg}^{-1}$ $\min ^{-1}$ during the remaining 26 cases because the NTG infusion rate of 3 $\mu \mathrm{g} \mathrm{kg}^{-1} \mathrm{~min}^{-1}$ was sometimes reached during short hypertensive periods in insensitive patients.

\subsubsection{Measurement protocol}

The closed-loop controller was introduced into the operating room after it had been extensively tested using a mathematical model which simulated a wide range of patient responses. The controller was generally switched to automatic mode before sternotomy and was switched to manual mode at the start of the cardiopulmonary bypass. The controller was switched back to automatic mode after cardiopulmonary bypass, if required. Target MAP was set between $65 \mathrm{mmHg}$ and $90 \mathrm{mmHg}$, depending on the patient's haemodynamic state and the surgical procedure. The controller's operation was closely supervised at all times by the anaesthesiologist and the system's designer.

Control cardiac output, arterial pressures and heart rate measurements were taken before the start of the operation (Control) and when the NTG infusion rate had stabilized (Stable-NTG). The time of a third set of haemodynamic data was determined offline by finding the maximum 30-second averaged MAP between Control and Stable-NTG (Max-MAP). For these calculations, arterial pressures and heart-rate values were averaged during 2 minutes to eliminate the influence of small fluctuations. Cardiac output was calculated using the technique developed by Jansen et al. (1990a) from a set of four thermodilution measurements taken during haemodynamically stable situations and at distances equally spread over the ventilatory cycle. Statistical analyses of the numerical data were carried out using analysis of variances (ANOVA) and a Bonferonni correction for multiple comparisons. All values are mean $\pm \mathrm{SD}$.

\subsection{Results}

Data for all 40 patients who responded to NTG were analysed. Two representative examples of the controller's action are given in figures 7.2 and 7.3 . Table 7.1 gives the haemodynamic data before the start of the operation, when MAP was maximum and when the NTG infusion had stabilized. Compared to control, arterial pressures rose significantly $(p<0.05)$, but returned to baseline values when the NTG infusion had stabilized. Cardiac 


\begin{tabular}{lccc}
\hline & Control & Max-MAP & Stable-NTG \\
\hline MAP (mmHg) & $82.6 \pm 13.6$ & $98.5 \pm 9.8^{*}$ & $81.4 \pm 8.3 \mathrm{NS}$ \\
$\mathrm{P}_{\text {art }} \mathrm{s}(\mathrm{mmHg})$ & $121.2 \pm 18.9$ & $143.5 \pm 17.5^{*}$ & $124.0 \pm 13.1 \mathrm{NS}$ \\
$\mathrm{P}_{\text {art }} \mathrm{d}(\mathrm{mmHg})$ & $62.6 \pm 12.1$ & $73.9 \pm 11.0^{*}$ & $62.8 \pm 8.2 \mathrm{NS}$ \\
$\mathrm{CO}\left(\mathrm{L} \mathrm{min}\right.$ m $\left.^{-1}\right)$ & $4.1 \pm 0.9$ & - & $4.8 \pm 1.2^{*}$ \\
$\mathrm{HR}$ (beats min & & -1 \\
${ }^{*} p<0.05$ compared with control & $61.8 \pm 14.7$ & $69.4 \pm 16.1^{*}$ & $73.2 \pm 18.5^{*}$ \\
NS, not significant compared with control &
\end{tabular}

Table 7.1: Haemodynamic data before the start of the operation (Control), when the 30-second averaged MAP was maximum (Max-MAP) and when the NTG infusion rate stabilized (Stable-NTG). Values are mean \pm SD. MAP, mean arterial pressure. $P_{a r t} s$, systolic arterial pressure. $P_{\text {art }} d$, diastolic arterial pressure. $C O$, cardiac output. HR, heart rate.

output and heart rate significantly $(\mathrm{p}<0.05)$ increased compared to control. Before cardiopulmonary bypass, the controller was in automatic mode for a total of 29.1 hours (average $44 \mathrm{~min}$ ). After cardiopulmonary bypass, the controller was used to control hypertension in 24 cases for a total of 17.7 hours (average $44 \mathrm{~min}$ ). In the remaining 16 cases, SNP was preferred after aortic valve replacement surgery $(n=6)$ or hypertension did not occur after cardiopulmonary bypass $(n=10)$. The NTG infusion rate while MAP was within $5 \mathrm{mmHg}$ of target MAP before and after cardiopulmonary bypass was, respectively, $1.36 \pm 0.92$ and $0.78 \pm 0.48 \mu \mathrm{g} \mathrm{kg}^{-1} \mathrm{~min}^{-1}$ (excluding the $0.5 \mu \mathrm{g} \mathrm{kg}^{-1} \mathrm{~min}^{-1} \mathrm{NTG}$ infusion in patients with a coronary bypass graft after cardiopulmonary bypass). The time necessary to bring the 30 -second averaged MAP to within $5 \mathrm{mmHg}$ of the target MAP at the start of the operation could be calculated in 26 cases and was $9.4 \pm 4.1$ min. In the remaining 14 cases, MAP was within $5 \mathrm{mmHg}$ of target MAP when the controller was switched to automatic mode or a smooth decrease in pressure towards target MAP was disturbed by the surgical procedure. The percentage of time that MAP remained in a range around the target MAP of $\pm 10 \mathrm{mmHg}$ and $\pm 20 \mathrm{mmHg}$ was respectively 74 and $94 \%$.

In all 40 cases the PI regulator was overruled 24 times by the supervisory computer program during a large unexpected MAP increase $(n=16)$ or decrease $(n=8)$. In four cases an additional bolus infusion of sufentanil $(n=3)$ or a temporary use of isoflurane $(n=1)$ was required at the start of the operation. In three other cases supplementary anaesthetics (isoflurane, alfentanil or sufentanil) were given during the procedure to counter an unexpectedly large increase in MAP. During all these episodes the controller was maintained in automatic mode and it was used to control 


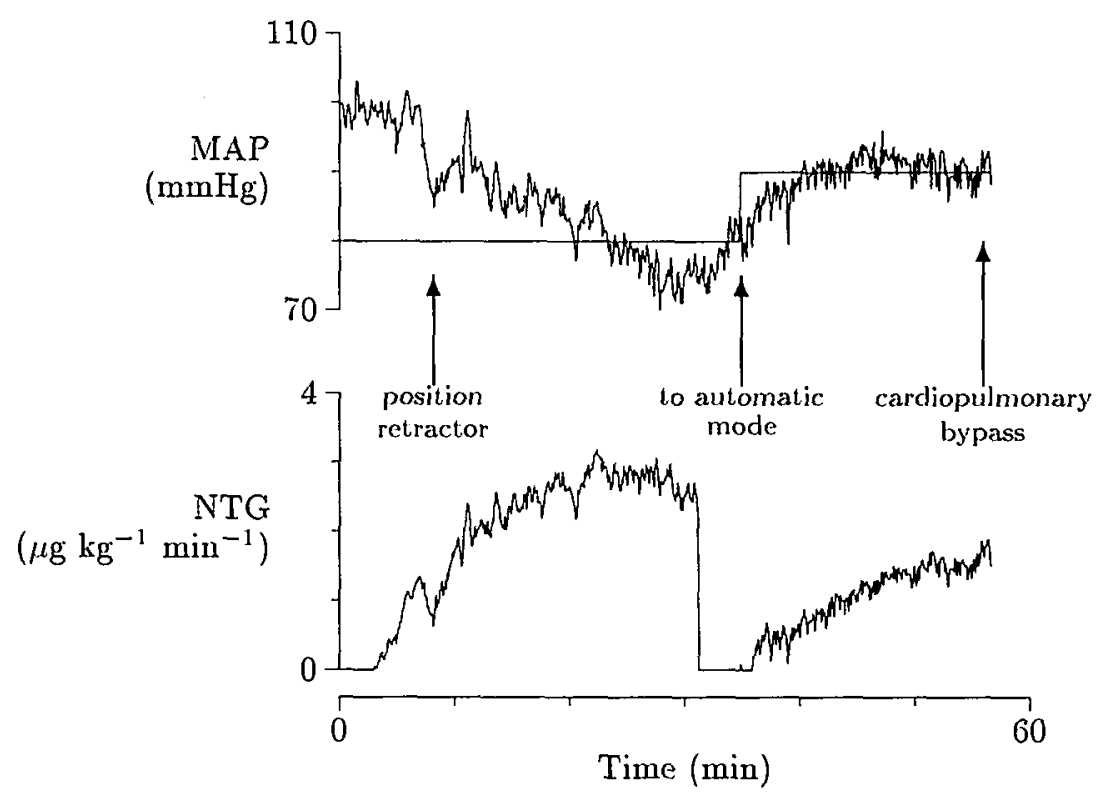

Figure 7.2: Example of the controller's performance during surgery. The controller is switched to automatic mode at the start of the operation. The positioning of the retractor causes the pressure to drop temporarily. After $30 \mathrm{~min}$, the nitroglycerin (NTG) infusion is stopped by the anaesthesiologist. A few minutes later the target mean arterial pressure (MAP) is increased and the controller is switched back to automatic mode. The system is stopped at the start of the cardiopulmonary bypass. 


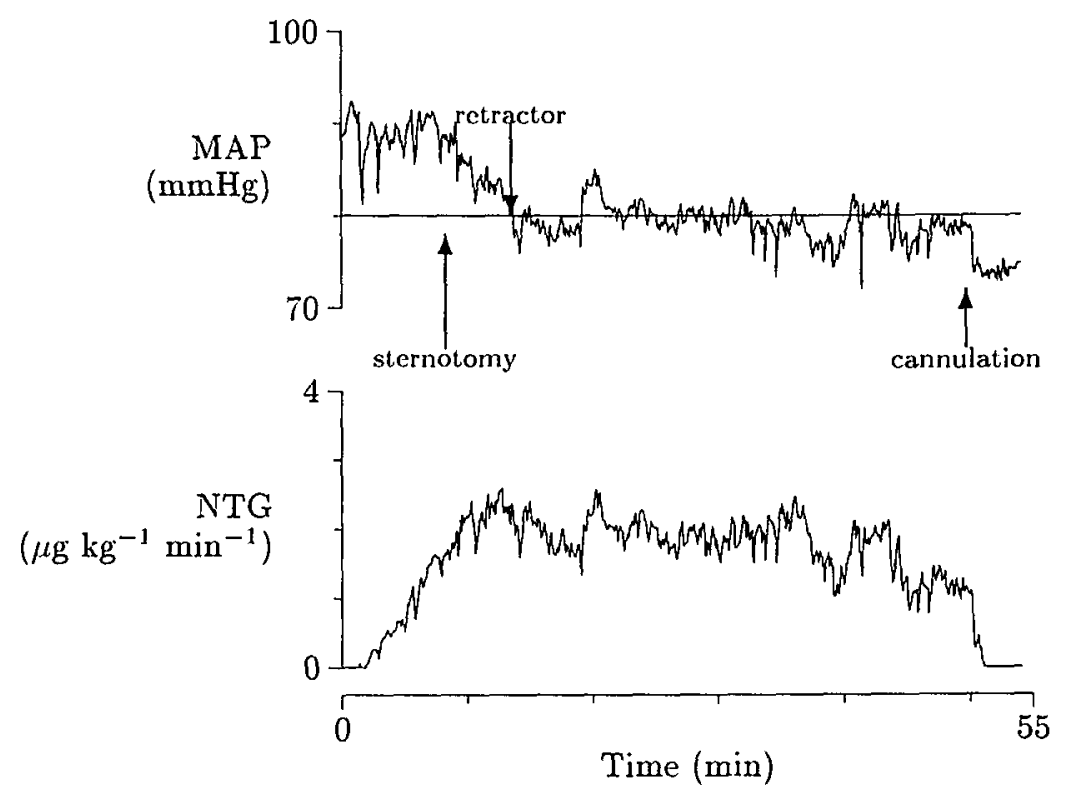

Figure 7.3: Example of the controller's performance during surgery. Sternotomy is performed at $8 \mathrm{~min}$. During the positioning of the retractor the pressure decreases slightly. The decrease in mean arterial pressure (MAP) caused by cannulation causes the controller to reduce the nitroglycerin (NTG) infusion rate to zero. 
the NTG infusion rate during the remaining time.

\subsection{Discussion}

Taking into account the lack of response to NTG in some patients, the controller appeared to be able to control MAP during cardiac surgery in the majority of patients while bringing arterial pressures back to the control values as measured before the start of the operation. Compared to control values cardiac output and heart rate increased slightly. The controller never caused a clinically dangerous situation nor was there any need to overrule its actions manually. The pressure validation algorithm preserved the normal actions of the arterial line such as flushing and the taking of blood samples and never caused the controller to respond to invalid pressure measurements. The controller never displayed unstable or oscillatory behaviour.

We preferred to use the vasodilator NTG, rather than SNP, for its positive effect on coronary perfusion and microcirculation (Chiariello et al. 1976; Hempelmann et al. 1977; Kaplan and Jones 1979; Endrich et al. 1987). NTG was found to be a suitable drug for use in a closed-loop system. A small group of patients (seven of the 40 ) required additional anaesthetics. During these episodes, it was not necessary to switch the closed-loop system back to manual mode and the system satisfactorily controlled MAP after a sufficient level of anaesthesia had been restored. There remained, however, a group of patients (six of the 46) who did not respond to NTG. Colvin and Kenny (1989) reported that 10 of their 24 postoperative patients required an additional infusion of SNP. Their aim, however, was to control systolic pressures. Similar results were obtained when NTG infusions were controlled manually (Csongrady and Ponz-Gonzalez-L 1980; Guggiari et al. 1985). We, therefore, conclude that an alternative vasodilator (e.g. SNP) should be kept on standby when controlling arterial pressure with NTG.

The lack of sufficient a priori knowledge about of the patient's response to vasodilator drug treatment is usually overcome by enabling the controller to learn from the patient response during a control session (Isaka and Sebald 1993). These adaptive controllers may satisfactorily control hypertension in the intensive care unit. During cardiac surgery, however, haemodynamics can change suddenly owing to surgical interferences and infusions of other drugs, inducing pressure fluctuations which are not correlated with the vasodilator infusion rate. The knowledge that can be derived from the arterial pressure signal is therefore limited during cardiac surgery. Martin 
et al. (1992b) used a multiple-model adaptive SNP controller to control arterial pressure during cardiac surgery. Their controller performs in a clinically acceptable manner but still requires manual adjustment of the SNP infusion rate at the onset of hypertension.

We did not attempt to identify the patient's response during the operation in our design. Instead, the supervisory program observed the control performance of the regulator. Control gain was decreased by the supervisory program when control was unstable, whereas it was increased when control could not be achieved. Besides the control gain adaptation rules, we modelled the expert's (i.e. the anaesthesiologist's) decision-making ability into the supervisory program. This implemented expert knowledge enabled the controller to exercise a form of common sense under all circumstances. It defined, among other things, the controller's response during sudden periods of extreme hypertension (figure 7.4) or hypotension. These transient changes in pressure can occur during cardiac surgery and are often caused by painful stimuli combined with insufficient anaesthetic levels or by reflex responses caused by manipulation of the heart or aorta.

A prerequisite for successful vasodilator treatment during surgery is an adequate level of anaesthesia. To ensure sufficient anaesthetic levels, patients were treated with a similar continuous infusion of opioids as used by Mangano et al. (1992). Bolus infusions of drugs were avoided wherever possible because they result in unstable haemodynamic profiles. Insufficient anaesthetic levels may result in haemodynamic instability or even in hypertension. In the presence of this hypertension, the controller will still try to lower the pressure and hence increase the NTG infusion rate. The anaesthesiologist should be aware that a change in the NTG infusion rate, even when MAP remains unchanged, may indicate a change in the patient's anaesthetic level. Therefore, in a closed-loop system, the value of MAP as an indicator of the anaesthetic level may be suppressed and even taken over by the NTG infusion rate. This side-effect of closed-loop control has been noted previously for both the postoperative (Hammond et al. 1979) and the intraoperative patient (Martin et al. 1992b).

Although closed-loop systems can satisfactorily control clinical variables, they should never be regarded as stand-alone devices, only as decision support systems. The anaesthesiologist will always have a better insight into the patient's condition and should therefore closely supervise the controller's actions. A clear (trend) display of the controller's output (NTG infusion rate) and haemodynamic variables is indispensable. Furthermore, the software safety of closed-loop systems also deserves special attention. Beatty (1993) correctly pointed out that patient-connected control systems 


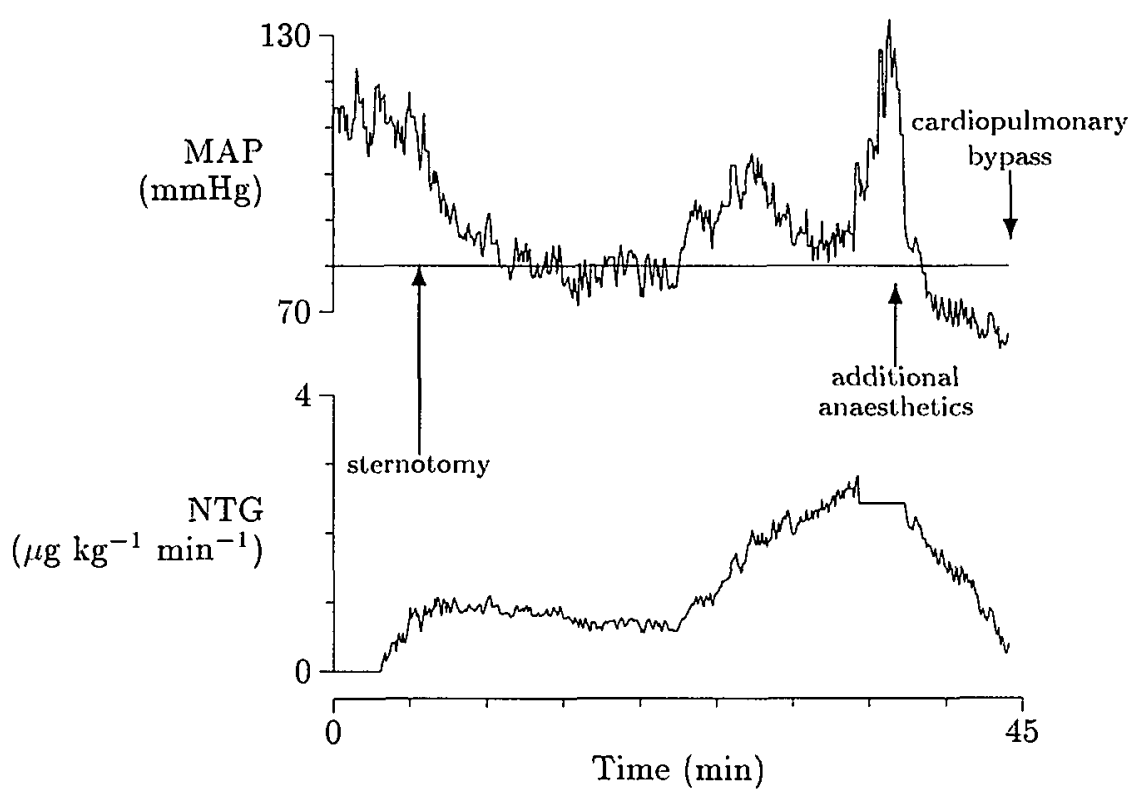

Figure 7.4: The controller's response to a large transient increase in mean arterial pressure (MAP). The controller was switched to automatic mode at the start of the operation. Sternotomy is performed at $6 \mathrm{~min}$. The pressure increases at $35 \mathrm{~min}$. Additional lorazepam and sufentanil were given at $37 \mathrm{~min}$. The supervisory program detected the pressure increase and kept the nitroglycerin (NTG) infusion rate constant until the end of the pressure increase was detected. Cardiopulmonary bypass starts at $44 \mathrm{~min}$. 
should follow strict safety guidelines.

We conclude that closed-loop control of arterial pressure is not only feasible in the cardiac surgical care unit, but also during cardiac surgery. NTG is a good vasodilator for this purpose, provided that the patient is not resistant to NTG. However, automated infusion of vasoactive drugs will be clinically successful only when the closed-loop system is integrated with the anaesthetic treatment of the cardiac surgical patient. It is to be expected that future research will demonstrate that the stable haemodynamic condition which can be achieved with these automated infusion devices will improve patient outcome not only for the postoperative patient (Chitwood et al. 1992), but also for the intraoperative patient. 


\title{
Chapter 8
}

\section{Automated infusion of sodium nitroprusside to control arterial hypertension during cardiac surgery}

\author{
S.A.A.P. Hoeksel \\ J. A. Blom \\ J. R. C. Jansen \\ J. G. Maessen \\ J. J. Schreuder
}




\section{Abstract}

Objective. To evaluate the feasibility of closed-loop control of systemic arterial hypertension during cardiac surgery using the vasoactive drug sodium nitroprusside.

Method. We previously described a control strategy for the control of arterial hypertension using the vasoactive drug nitroglycerin. We have investigated in the present study whether a similar control strategy can be used for the vasoactive drug sodium nitroprusside. Compared to nitroglycerin, sodium nitroprusside has a shorter half-life time and stronger variations in patient sensitivity between individuals. The control strategy was adjusted for these differences in pharmacodynamics. The sodium nitroprusside blood-pressure controller was used to control systemic arterial hypertension in 40 patients during cardiac surgery.

Results. The anaesthesiologist never manually overruled the decisions taken by the closed-loop system; we therefore conclude that control was stable and sufficiently fast in all patients. The time necessary to bring the 30 second averaged mean arterial pressure to within $5 \mathrm{mmHg}$ of the target could be calculated in eight cases and was $7.6 \pm 1.9$ minutes (mean \pm SD). The percentage of time that mean arterial pressure remained in a range of \pm 10 and $\pm 20 \mathrm{mmHg}$ was respectively 76 and $93 \%$. The mean sodium nitroprusside infusion rate when mean arterial pressure was within $5 \mathrm{mmHg}$ of the target before and after cardiopulmonary bypass was respectively $1.20 \pm 0.83$ and $0.67 \pm 0.59 \mu \mathrm{g} \mathrm{kg}^{-1} \mathrm{~min}^{-1}$.

Conclusions. Closed-loop control of systemic arterial hypertension using sodium nitroprusside is feasible during cardiac surgery. Unlike nitroglycerin, the vasoactive drug sodium nitroprusside effectively lowered arterial pressures in all patients.

Keywords sodium nitroprusside - computer control • cardiac surgery • hypertension 


\subsection{Introduction}

Most closed-loop systems applied clinically have been designed for the control of postoperative systemic hypertension in cardiac surgical patients (O'Hara et al. 1992). The vasoactive drug that is most commonly used in these studies is sodium nitroprusside (SNP) (Sheppard 1980; Chitwood et al. 1992; Ruiz et al. 1993), although the use of nitroglycerin (NTG) has also been reported (Colvin and Kenny 1989). Fewer studies have reported on the use of a closed-loop blood-pressure controller during cardiac surgery. Martin et al. (1992b) evaluated a multiple-model adaptive SNP blood-pressure controller during cardiac surgery. Their system, however, required manual adjustments of the SNP infusion rate at the onset of hypertension. Blom (1991) evaluated an adaptive SNP blood-pressure controller during cardiac surgery, but concluded that the system's cautious behaviour made it less ideal for the operating room. Meijers et al. (1997) recently described an improvement in the system that was originally described by Blom (1991) and evaluated it during cardiac surgery in 33 patients, claiming an acceptable control performance in all patients.

In chapter 7 the clinical feasibility of a NTG blood-pressure controller was established. NTG proved to be effective in most patients, but there remained a small group (six out of 46) of patients who did not respond to NTG and required treatment with SNP. Furthermore, in patients with minimal cardiac preload reserve use of SNP rather than NTG is indicated (Kaplan and Guffin 1993). For these reasons we investigated whether the NTG control strategy described in chapter 7 could also be used for the control of SNP infusion rates.

\subsection{Material and method}

\subsubsection{Patients}

The SNP blood-pressure controller was used during cardiac surgery in 40 patients who required SNP for the treatment of systemic arterial hypertension. The study was approved by our hospital's medical ethics committee. Informed patient consent was obtained on the evening before surgery. $\mathrm{Pa}$ tients were scheduled either for coronary bypass surgery ( 35 cases), valvular surgery (three cases) or for surgical repair of an aneurysmatic aorta (two cases). Most patients were male (29 out of 40 ), aged 34 to 76 years (average 62 years). Patients' weight ranged between 61 and $130 \mathrm{~kg}$ (average $82 \mathrm{~kg}$ ). Perioperative anaesthetic management did not differ from the protocol that 
was used during the closed-loop control of arterial hypertension using NTG (section 7.2.2, page 88).

\subsubsection{Control system}

The control system comprised a proportional-integral regulator which updated the infusion rate of a computer-controlled infusion pump (IVAC P4000, IVAC corporation) every five seconds. Values for the model parameters of the patient response to SNP were taken over from previously published data (Sheppard 1980; Blom 1990) (table 6.1, page 78). Compared to NTG, SNP has a shorter time constant, $\tau$ and larger variations in patient sensitivity, $K$, between individuals. The proportional-integral regulator and the gain adaptation mechanisms were adjusted to the pharmacodynamics of SNP. The infusion rate of SNP was limited to $3 \mu \mathrm{g} \mathrm{kg}^{-1}$ $\mathrm{min}^{-1}$, which is considered to be a safe upper limit in clinical practice and limits the chance of toxicity by elevated cyanide levels.

\subsubsection{Measurement protocol}

The SNP blood-pressure controller was introduced to the operating room after it had extensively been tested on a mathematical model which simulated a wide range of patient responses. The blood-pressure controller was generally switched to automatic mode at the start of the operation and remained in automatic mode until the start of the cardiopulmonary bypass. If required, the controller was switched back to automatic mode after cardiopulmonary bypass. Target MAP was generally set between 65 and 90 $\mathrm{mmHg}$. The controller's operation was closely supervised at all times by the anaesthesiologist and the system's designer.

Cardiac output, arterial pressures and heart-rate measurements were taken before the start of the operation (Control) and some time after sternotomy as soon as haemodynamics had stabilized (Stable-SNP). The time of a third set of haemodynamic data was determined offline by finding the maximum 30-second averaged MAP between Control and Stable-SNP (Max-MAP). Cardiac output was calculated using the technique developed by Jansen et al. (1990a) from a set of four thermodilution measurements taken at distances equally spread over the ventilator cycle. If required, additional cardiac output measurements were taken at haemodynamically stable intervals. For these calculations, arterial pressure and heart-rate values were averaged over 2 minutes to eliminate the influence of random fluctuations. 

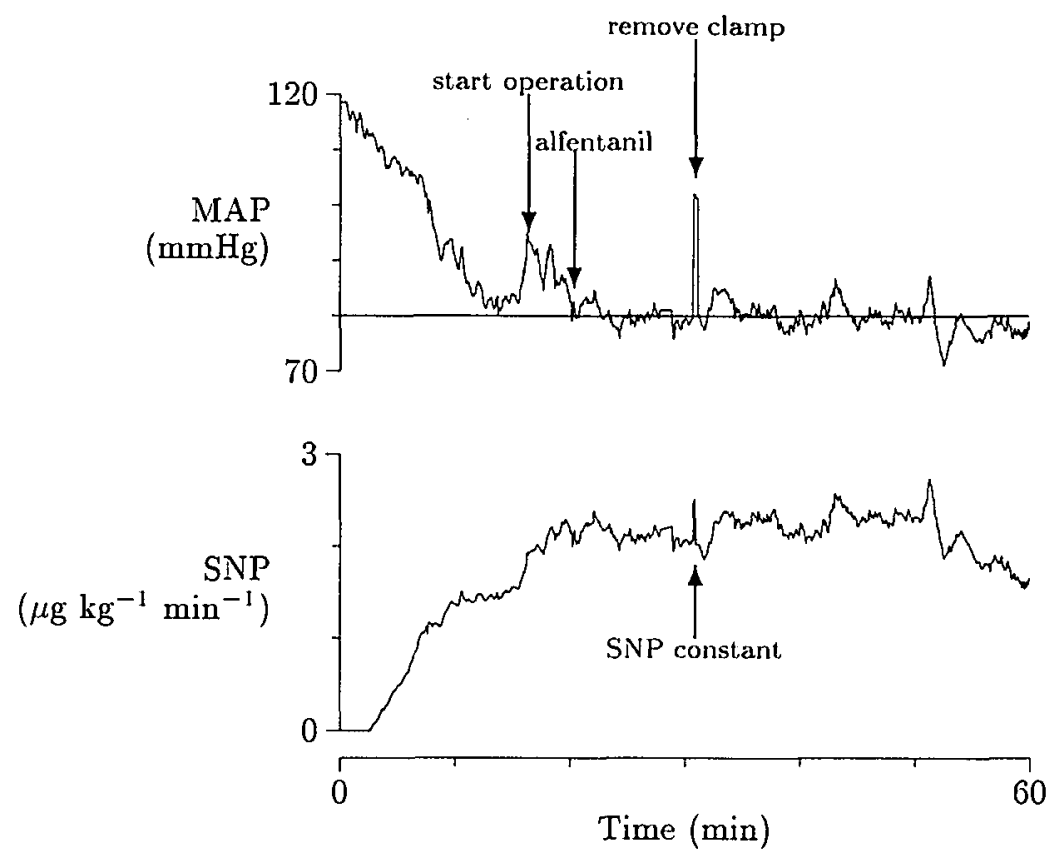

Figure 8.1: Recordings of a 48-year-old male patient scheduled for coronary artery bypass surgery. The sudden increase in mean arterial pressure (MAP) at $30 \mathrm{~min}$ was caused by the removal of a mammary artery clamp. The sodium nitroprusside (SNP) infusion rate initially increased in response to this MAP increase. A few seconds later the supervisory computer program detected the increase in MAP and kept the SNP infusion rate constant at the rate that was used prior to the increase in MAP.

Statistical analyses of the numerical data were caried out using analysis of variances (ANOVA) and a Bonferonni correction for multiple comparisons. All values are mean $\pm \mathrm{SD}$.

\subsection{Results}

The SNP blood-pressure controller was fast enough and provided stable control without the need to overrule the controller's actions manually. Illustrative examples of the controller's performance are given in figures 8.1 and 8.2. In all 40 patients the controller was used to control arterial hypertension before cardiopulmonary bypass for a total of 39 hours (average 59 minutes). After cardiopulmonary bypass, the controller was used to con- 

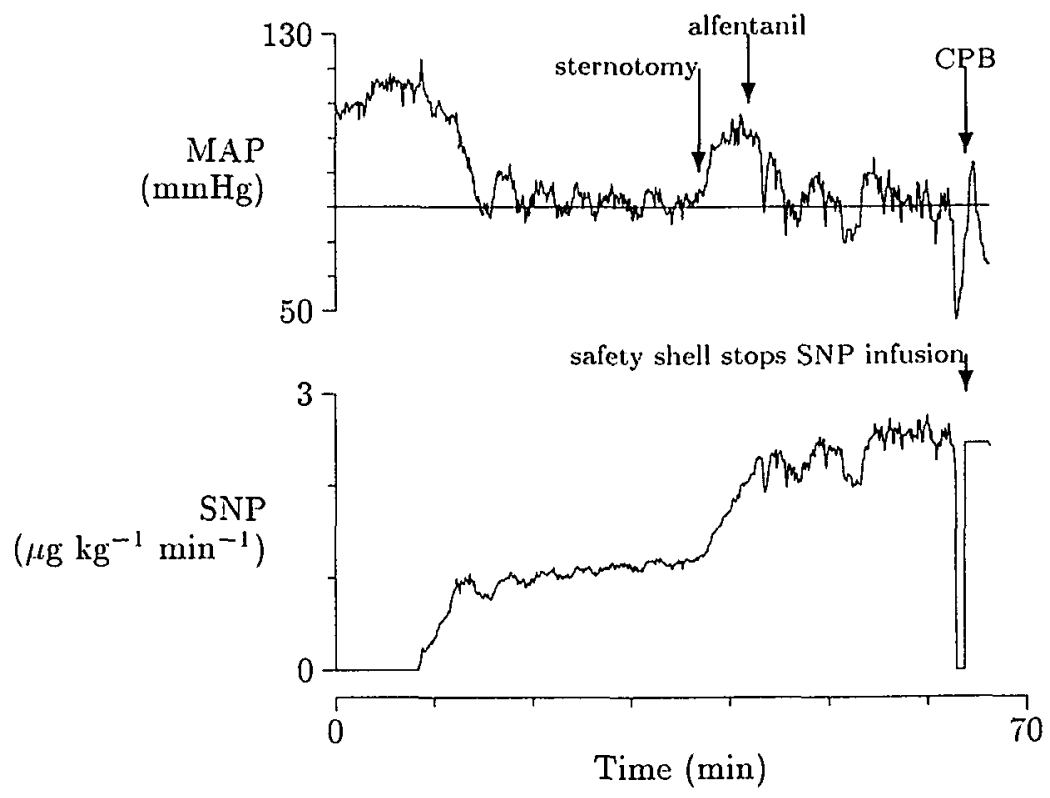

Figure 8.2: Recordings of a 60-year-old male patient scheduled for coronary bypass surgery. The sudden pressure drop caused by the cannulation prior to the cardiopulmonary bypass was detected by the supervisory computer program; the sodium nitroprusside (SNP) infusion rate was stopped till the end of the unexpected drop in mean arterial pressure (MAP) was detected. After cardiopulmonary bypass no SNP infusion was needed, but the oscillatory behaviour that is visible in the MAP was still present, indicating that oscillations before cardiopulmonary bypass were not induced by the closed-loop system.

\begin{tabular}{lccc}
\hline & Control & Max-MAP & Stable-SNP \\
\hline MAP (mmHg) & $86.2 \pm 12.8$ & $98.5 \pm 9.9^{*}$ & $82.9 \pm 8.7 \mathrm{NS}$ \\
$\mathrm{P}_{\text {art }}(\mathrm{mmHg})$ & $131.3 \pm 20.8$ & $151.9 \pm 17.0^{*}$ & $133.3 \pm 17.5 \mathrm{NS}$ \\
$\mathrm{P}_{\text {art }} \mathrm{d}(\mathrm{mmHg})$ & $64.8 \pm 10.1$ & $72.7 \pm 8.7^{*}$ & $62.2 \pm 7.2 \mathrm{NS}$ \\
$\mathrm{CO}\left(\mathrm{L} \mathrm{min}{ }^{-1}\right)$ & $4.7 \pm 1.4$ & - & $5.4 \pm 1.3^{*}$ \\
$\mathrm{HR}($ beats min & -1 \\
\hline${ }^{*} p<0.05$ compared with control & $64.2 \pm 12.3$ & $68.9^{*} \pm 15.9$ & $73.4 \pm 19.0^{*}$ \\
$\mathrm{NS}$, not significant compared with control &
\end{tabular}

Table 8.1: Haemodynamic data at the start of the operation (Control), when the 30-second averaged MAP was maximum (Max-MAP) and when the SNP infusion rate stabilized (Stable-SNP). Values are mean \pm SD. MAP, mean arterial pressure. $\mathrm{P}_{\text {art }} \mathrm{s}$, systolic arterial pressure. $\mathrm{P}_{\text {art }} \mathrm{d}$, diastolic arterial pressure. $\mathrm{CO}$, cardiac output. HR, heart rate. 
trol hypertension in 28 cases for a total of 26 hours (average 55 minutes). In the remaining 12 cases, hypertension did not occur after cardiopulmonary bypass. The SNP infusion rate while MAP was within $5 \mathrm{mmHg}$ of the target MAP before and after cardiopulmonary bypass was respectively $1.20 \pm 0.83$ (mean $\pm \mathrm{SD}$ ) and $0.67 \pm 0.59 \mu \mathrm{g} \mathrm{kg}^{-1} \mathrm{~min}^{-1}$. Compared to control measurements, cardiac output and heart rate increased (table 8.1). Arterial pressures rose significantly (Max-MAP), but returned to control levels as soon as the haemodynamics had stabilized (Stable-SNP) (table 8.1).

The time necessary to bring the 30 -second averaged MAP to within 5 $\mathrm{mmHg}$ of target MAP could be calculated in eight cases and was $7.6 \pm 1.9$ min. In the remaining 32 cases, MAP was within $5 \mathrm{mmHg}$ of the target MAP when the controller was switched to automatic mode or a smooth decrease in pressure towards the target MAP was disturbed by the surgical procedure. The percentages of time that MAP remained in a range around target MAP of \pm 10 and $\pm 20 \mathrm{mmHg}$ was respectively 76 and $93 \%$. In all 40 cases the proportional-integral regulator was overruled 29 times by the supervisory computer program during a large unexpected MAP increase (in 21 cases) or decrease (in eight cases). MAP decreased in response to SNP in all patients, although in six patients the SNP infusion rate reached its maximum value of $3 \mu \mathrm{g} \mathrm{kg}^{-1} \mathrm{~min}^{-1}$ and in one of these six patients MAP could not be lowered to its target; for this patient the anaesthesiologist considered the final MAP level acceptable. In 14 patients additional boluses of sufentanil or alfentanil (13 cases) or a temporary use of isoflurane (one case) were required before cardiopulmonary bypass. In six patients additional anaesthetics (five cases) or temporary use of isoflurane (one case) were required after cardiopulmonary bypass. During all these episodes the controller was kept in automatic mode.

\subsection{Discussion}

The SNP blood-pressure controller satisfactorily controlled arterial hypertension in all 40 patients while bringing MAP slightly below the control values as measured before the start of the operation (table 8.1). Compared to the NTG patient group (table 7.1, page 92), the SNP patient group had somewhat higher pressure values at the start of the operation, indicating probably the underlying differences in selection criteria for both patient groups. The control performance of the MAP-SNP controller, as derived from the percentages of time that MAP remained inside a range around the target MAP, was comparable to control performance of the MAP-NTG 
controller (chapter 7), although the time necessary to reach target MAP appeared to be somewhat shorter for the MAP-SNP controller (7.6 versus $9.4 \mathrm{~min}$ ).

The most frequently used drugs for the control of intraoperative arterial hypertension are SNP and NTG. Compared to NTG, SNP proved to be a more effective drug in treating systemic arterial hypertension, because, unlike NTG, SNP effectively lowered arterial pressures in all patients. Which vasodilator is preferred for which patient also depends, however, on the haemodynamic state of the patient. In the ischaemic patient, for example, NTG is often preferred to SNP, because SNP decreases coronary perfusion pressures and might cause cardiac ischaemia (Kaplan and Jones 1979).

Chitwood et al. (1992) used a SNP blood-pressure controller to control postoperative hypertension in cardiac surgical patients and found a significant improvement in patient outcome. The present study and the recent paper by Meijers et al. (1997) demonstrate that closed-loop control of arterial hypertension using SNP is also feasible during cardiac surgery. Future research, preferably in a multi-centre setting, should however investigate the effects of closed-loop blood-pressure control during cardiac surgery on patient outcome. 


\section{Chapter 9}

\section{Automated infusion of nitroglycerin to control pulmonary hypertension during cardiac surgery}

S. A. A. P. Hoeksel

J. A. Blom

J. R. C. Jansen

J. G. Maessen

J. J. Schreuder 


\section{Abstract}

Objective. To evaluate the feasibility of closed-loop control of pulmonary artery hypertension during cardiac surgery.

Method. A closed-loop system for the control of pulmonary hypertension has been developed based on a previously described control strategy. Before validation the pulmonary artery pressure signal was corrected for the cyclic ventilator artefact by a least squares fitting procedure. The closed-loop system was evaluated in 11 patients who required nitroglycerin for the treatment of elevated pulmonary artery pressures during cardiac surgery. Results. The closed-loop system provided safe and sufficiently fast control in all patients. The NTG infusion rate while the mean pulmonary artery pressure was within $2 \mathrm{mmHg}$ of the target before and after cardiopulmonary bypass was respectively $1.46 \pm 0.89$ and $1.26 \pm 0.81 \mu \mathrm{g} \mathrm{kg}^{-1} \mathrm{~min}^{-1}$. The percentages of time that the mean pulmonary artery pressure remained in a range of \pm 5 and $\pm 10 \mathrm{mmHg}$ around its target was respectively 76 and $95 \%$. The pressure validation algorithm did not once interpret a disturbed pulmonary artery pressure signal as valid, although with atrial fibrillation some pressure measurements were incorrectly labelled as invalid.

Conclusions. Closed-loop control of pulmonary artery hypertension using nitroglycerin appeared to be feasible during cardiac surgery.

Keywords nitroglycerin $\bullet$ cardiac failure $\bullet$ pulmonary hypertension $\bullet$ computer control $\bullet$ cardiac surgery 


\subsection{Introduction}

Management of circulatory filling in cardiac surgical patients is usually guided by the level of the pulmonary wedge pressure. Wedge pressure can however not be measured continuously and therefore, in the absence of pulmonary problems, the mean pulmonary artery pressure (MPAP) is often used. Too low MPAP is treated by infusion of additional volume. Too high MPAP is treated by a venodilator drug, such as nitroglycerin (NTG), to relieve the preload of the ventricles and prevent decompensation cordis. One of the first closed-loop systems that was used in a clinical setting automated the infusion of blood in postoperative cardiac surgical patients (Sheppard et al. 1968), but as far as we have been able to establish there have been no attempts to closed-loop control a situation of too high circulatory filling.

Control of elevated MPAP in the presence of left ventricular heart failure or mitral valve defects requires a repetitive adjustment of the NTG drug infusion rate. We investigated whether this repetitive adjustment can be taken over by a closed-loop system. The control strategy was based on a previously described control strategy for the control of systemic arterial hypertension (chapters 7 and 8).

\subsection{Material and method}

\subsubsection{Patients}

MPAP was controlled in 11 patients who were scheduled for cardiac surgery and had pulmonary hypertension secondary to left ventricular heart failure or mitral valve defects. Six of the patients were scheduled for coronary bypass surgery and five for valvular surgery. Most patients were female (six out of 11), aged 51 to 74 years (average 65 years). Patients' weights ranged between 51 and $88 \mathrm{~kg}$ (average $71 \mathrm{~kg}$ ). The study was approved by our hospital's medical ethics committee. Informed patient consent was obtained on the evening before surgery. The anaesthetic protocol did not differ from the protocol that was used during the closed-loop control of systemic hypertension (section 7.2.2, page 88).

\subsubsection{Controller}

The MPAP-NTG control system comprised a proportional-integral regulator which updated the infusion rate of a computer-controlled infusion 
pump (IVAC P4000) every five seconds. A supervisory computer program adapted or overruled the proportional-integral regulator when necessary. The patient model parameter values (table 6.1 , page 78 ) that were used to tune the proportional-integral regulator and the gain adaptation mechanisms were based on our experience with closed-loop control of systemic arterial hypertension using NTG (chapter 7). Unlike the closed-loop systems for systemic arterial hypertension (chapters 7 and 8), the MPAPNTG regulator is not overruled by the supervisory safety shell during sudden increases or decreases in MPAP, because sudden increases in MPAP are routinely treated by vasodilator drugs provided an adequate depth of anaesthesia has been established.

The quality of the pulmonary pressure signal was verified by comparing several features of each pulmonary pressure beat with a moving average model of the features of the previous pressure beats (chapter 3 ). The pulmonary pressure features were corrected for the respiration artefact by fitting them in a least squares sense on the first and second harmonics of the ventilator frequency (chapter 3 ).

\subsubsection{Measurement protocol}

The MPAP-NTG controller was introduced into the operating room after it had been extensively tested on a mathematical model that simulated a wide range of patient responses. The MPAP-NTG controller was used to control pulmonary hypertension before and after cardiopulmonary bypass. Target MPAP was generally set between 20 and $25 \mathrm{mmHg}$. The controller's operation was closely supervised at all times by the anaesthesiologist and the system's designer.

Cardiac output, pulmonary artery and systemic pressure and heart rate measurements were taken at the start (Start-Oper) of the operation, before (Start-Bypass) and after cardiopulmonary bypass (End-Bypass) and at the termination of the operation (End-Oper). If required, additional cardiac output measurements were made during the procedure at haemodynamically stable intervals. Cardiac output was calculated using a technique developed by Jansen et al. (1990a), from a set of four thermodilution measurements taken at distances equally spread over the ventilator cycle. For these calculations, pressure and heart-rate measurements were averaged over two minutes to eliminate the influence of random fluctuations. 

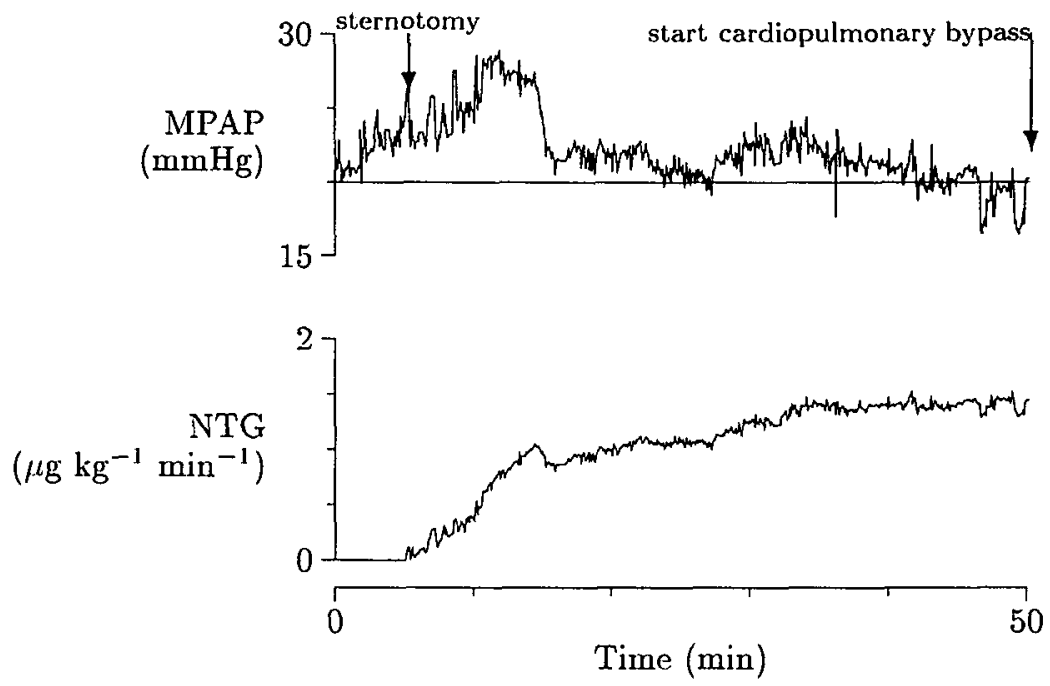

Figure 9.1: Recordings of a 63-year-old male patient scheduled for coronary bypass surgery. An intra aortic balloon pump was used in this patient. MPAP, mean pulmonary artery pressure. NTG, nitroglycerin.

\subsection{Results}

The MPAP-NTG controller was used to control pulmonary hypertension before and after cardiopulmonary bypass for a total of respectively 9.2 hours (average 50 minutes) and 9.5 hours (average 52 minutes). Illustrative examples of the MPAP-NTG controller are given in figures 9.1 and 9.2. Results from this limited group of patients indicated that control was stable and adequately fast. There was never any need to overrule the controller's actions manually. Table 9.1 shows the haemodynamic data measured during the procedure. Both before and after cardiopulmonary bypass, NTG lowered pulmonary pressures whilst arterial pressures remained unchanged (table 9.1). After the infusion of NTG had started MPAP decreased in all 11 patients, although in two patients the maximum infusion rate of $5 \mu \mathrm{g}$ $\mathrm{kg}^{-1} \mathrm{~min}^{-1}$ was reached and in one of these two patients MPAP could not be lowered to target MPAP. The NTG infusion rate while MPAP was within $2 \mathrm{mmHg}$ of target MPAP before and after cardiopulmonary bypass was respectively $1.46 \pm 0.89$ and $1.26 \pm 0.81 \mu \mathrm{g} \mathrm{kg}^{-1} \mathrm{~min}^{-1}$. The percentage of time that MPAP remained in a range around the target MPAP of \pm 5 and $\pm 10 \mathrm{mmHg}$ was respectively 76 and $95 \%$.

The pulmonary pressure signal validation routine correctly interpreted 

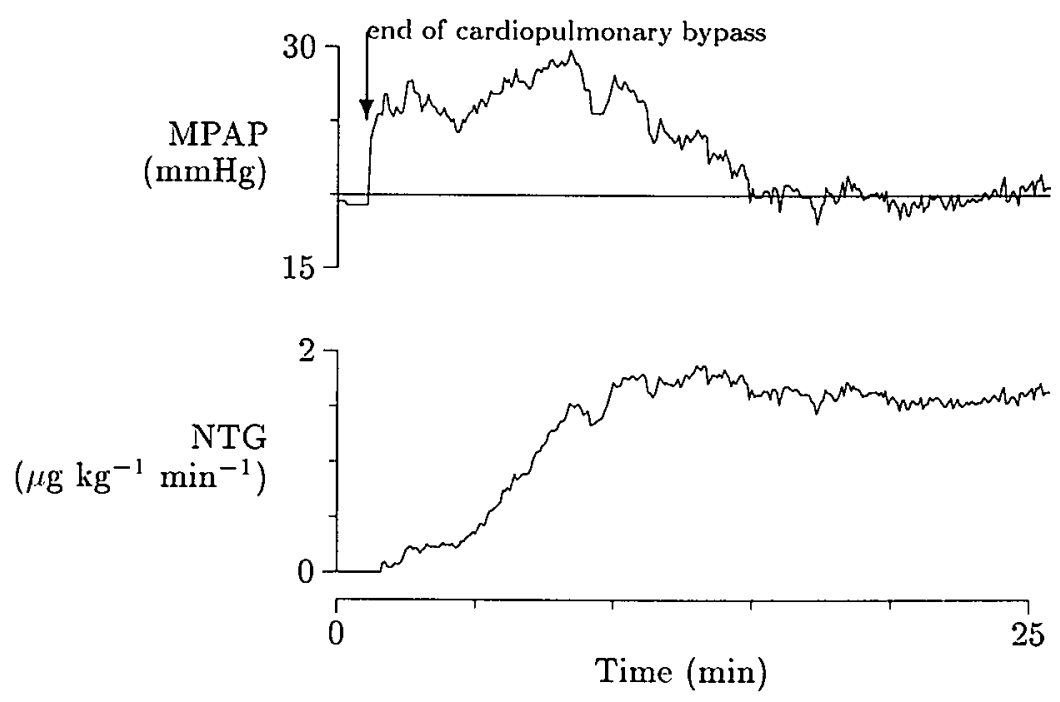

Figure 9.2: Recordings of a 61-year-old male patient scheduled for re-do coronary bypass surgery. The closed-loop system was switched to automatic mode at the end of the cardiopulmonary bypass. MPAP, mean pulmonary artery pressure. NTG, nitroglycerin.

\begin{tabular}{lcccc}
\hline & Start-Oper & Start-Bypass & End-Bypass & End-Oper \\
\hline MAP & $73.1 \pm 9.9$ & $69.1 \pm 12.3$ & $67.0 \pm 10.8$ & $70.6 \pm 7.0$ \\
$\mathrm{P}_{\text {arl }} \mathrm{S}$ & $106.2 \pm 19.9$ & $105.5 \pm 16.9$ & $96.7 \pm 24.0$ & $106.5 \pm 10.7$ \\
$\mathrm{P}_{\text {art }} \mathrm{d}$ & $51.8 \pm 9.0$ & $51.8 \pm 9.0$ & $48.5 \pm 8.9$ & $55.5 \pm 6.7$ \\
$\mathrm{MPAP}$ & $25.0 \pm 5.5$ & $20.9 \pm 4.3$ & $27.0 \pm 5.9$ & $22.7 \pm 2.8$ \\
$\mathrm{P}_{\text {pul }} \mathrm{S}$ & $32.9 \pm 7.2$ & $28.3 \pm 5.8$ & $33.8 \pm 10.6$ & $29.5 \pm 3.6$ \\
$\mathrm{P}_{\text {puld }}$ & $18.8 \pm 5.0$ & $16.2 \pm 4.0$ & $19.1 \pm 5.6$ & $17.5 \pm 3.4$ \\
$\mathrm{CO}$ & $3.8 \pm 0.7$ & $4.5 \pm 1.3$ & $5.2 \pm 1.2$ & $4.4 \pm 1.0$ \\
$\mathrm{HR}$ & $70.4 \pm 21.6$ & $83.6 \pm 15.9$ & $85.4 \pm 17.3$ & $85.8 \pm 10.7$ \\
\hline All units in mmHg, except CO in L min $^{-1}$ and HR in beats min
\end{tabular}

Table 9.1: Haemodynamic data at the start of the operation (Start-Oper), before (Start-Bypass) and after cardiopulmonary bypass (End-Bypass) and at the end of the operation (End-Oper). Values are mean \pm SD. MAP, mean arterial pressure. $\mathrm{P}_{\mathrm{art}} \mathrm{s}$, systolic arterial pressure. $\mathrm{P}_{\mathrm{art}} \mathrm{d}$, diastolic arterial pressure. MPAP, mean pulmonary artery pressure. $P_{p u l} s$, systolic pulmonary pressure. $P_{p u l} d$, diastolic pulmonary pressure. $\mathrm{CO}$, cardiac output. $\mathrm{HR}$, heart rate. 
the cyclic ventilator disturbances and never incorrectly labelled invalid pressure signals as valid. With three patients with atrial fibrillation the closedloop system sometimes switched back to manual mode (in total five times), because insufficient pressure beats were judged as valid by the validation routine. In these situations the control system alerted the anaesthesiologist, who, after verification of the quality of the pressure signal, switched the control system back to automatic mode.

\subsection{Discussion}

In chapters 7 and 8 it was demonstrated that a proportional-integral regulator combined with a supervisory safety shell can satisfactorily control arterial hypertension during cardiac surgery. Results from the present study indicate that a similar control strategy can be used for the control of pulmonary hypertension. Control of MPAP was stable and appeared fast enough in this limited patient group. Moreover, NTG effectively lowered MPAP in all patients. Both properties are important because marked increases in MPAP might indicate, when pulmonary problems are excluded, malfunctioning of the mitral valve or left ventricular decompensation. In both haemodynamic states MPAP needs to be lowered immediately during cardiac surgery. In this limited patient group, mean arterial pressure did not decrease in response to NTG, indicating a different effect of NTG in patients with cardiac failure than in patients with systemic hypertension (chapter 7 ).

This study demonstrated that computer control of a pulmonary hypertension is feasible during cardiac surgery. Future research should investigate whether control of a situation of low circulatory filling is feasible. not only for the postoperative patient (Sheppard et al. 1968), but also for the intraoperative patient. 


\section{Chapter 10}

Automated infusion of vasoactive and inotropic drugs to control arterial and pulmonary pressures during cardiac surgery

S. A. A. P. Hoeksel

J. A. Blom

J. R. C. Jansen

J. G. Maessen

J. J. Schreuder 


\begin{abstract}
Objective. To evaluate the feasibility of a closed-loop system for the simultaneous control of systemic arterial and pulmonary artery blood pressures during cardiac surgery.

Method. A multiple-drug closed-loop system integrated five single-drug blood-pressure controllers. Arterial hypertension was controlled using sodium nitroprusside or nitroglycerin, arterial hypotension was controlled using noradrenalin or dobutamine and pulmonary hypertension was controlled using nitroglycerin. The anaesthesiologist selected target pressures and single-drug blood-pressure controllers. The multiple-drug closed-loop system had a set of priority rules that automatically activated from the selected single-drug controllers the optimum single-drug controller for each haemodynamic state. Drug infusion rates of the non-active controllers were kept constant. The initial knowledge that was used to construct the priority rules was obtained from standard anaesthetic protocols on perioperative management of cardiac surgical patients. A supervisory safety shell defined the actions to be taken in cases of infusion pump problems, invalid pressure measurements and during unexpected increases and decreases in systemic arterial pressure. The performance of the multiple-drug closed-loop system was evaluated during cardiac surgery in 30 patients who required treatment with more than one vasoactive or inotropic drug.

Results. The activation of single-drug controllers by the priority rules was accurate and fast. On average a different single-drug controller was activated once every $7.2 \mathrm{~min}$. The average deviation of mean arterial pressure and mean pulmonary artery pressure from their target values was respectively $8.6 \pm 4.0$ and $4.4 \pm 4.0 \mathrm{mmHg}$ before cardiopulmonary bypass and $8.0 \pm 3.6$ and $2.4 \pm 0.9 \mathrm{mmHg}$ after cardiopulmonary bypass. None of the single-drug controllers ever showed any sign of unstable behaviour.

Conclusion. Closed-loop control of both arterial and pulmonary pressures using multiple drugs is feasible during cardiac surgery.
\end{abstract}

Keywords computer control • multiple drug delivery • cardiac surgery 


\subsection{Introduction}

Closed-loop control of haemodynamics was first reported by Sheppard et al. (1968) who automated the infusion of blood to control atrial pressures in postoperative cardiac surgical patients. The continuing interest in closedloop control has been illustrated in various articles (Katona 1982; Linkens and Hacisalihzade 1990; O'Hara et al. 1992). Most closed-loop systems automate the infusion of a vasoactive drug to control systemic arterial hypertension in postoperative patients. Recently, we showed that closedloop control of systemic arterial hypertension is also feasible during cardiac surgery (chapters 7 and 8 ). Chitwood et al. (1992) showed that, compared with manual control, closed-loop control of postoperative hypertension significantly improves patient outcome, by reducing transfusion requirements and postoperative blood loss and by decreasing the number of hypertensive and hypotensive periods. Despite these improvements in patient outcome, closed-loop control of blood pressure is not readily accepted in routine clinical practice. One explanation might be that control of blood pressure using a single vasoactive drug does not control overall haemodynamics. Especially in the haemodynamically unstable (heart-failure) patient, repeated adjustment of vasoactive and inotropic drug infusion rates is needed to maintain cardiac output and left ventricular preload and afterload within an acceptable range (Kaplan and Cruffin 1993). It is worthwhile to investigate the feasibility of closed-loop control of overall haemodynamics in haemodynamically unstable patients, because it is specifically these patients that might benefit the most from closed-loop control.

The closed-loop control of haemodynamics requires an adaptive control strategy (Isaka and Sebald 1993), because patient response varies widely between patients and within one patient over time. Voss et al. (1987) used a minimum variance controller to control arterial hypertension and cardiac output in dogs simultaneously. Yu et al. (1992) evaluated a similar strategy in dogs, but used multiple-model adaptive control to limit the estimated model to a finite set of models. Held and Roy (1995) used computer simulations to evaluate a fuzzy-logic controller for the simultaneous control of cardiac output and arterial hypertension. The feasibility of closed-loop control schemes that vary more than one drug infusion rate at the same time is however questionable, because differentiation between drug effects is difficult. In clinical practice continuous reliable identification of the multiple-input multiple-output patient model can therefore not be guaranteed, resulting in a potentially unstable control clesign. We observed, however, that in practice the anaesthesiologist tries to avoid si- 
multaneous changes in drug infusion rates by controlling the most critical haemodynamic variables first. We therefore speculated that the problem of closed-loop control with multiple drugs can be reduced to the design of a set of priority rules that selects the optimum single-drug controller for a given haemodynamic state.

This report describes the integration of five single-drug blood-pressure controllers into a single multiple-drug control system. The single-drug systems controlled arterial hypertension using sodium nitroprusside (SNP) or nitroglycerin (NTG), arterial hypotension using noradrenaline (NAD) or dobutamine (DOBU) and pulmonary hypertension using NTG. A set of priority rules continuously analysed the patient's haemodynamic state and automatically activated the optimum single-drug controller. The drug infusion rates of the non-active controllers were kept constant. We did not include the control of cardiac output, because we felt that the current techniques to monitor cardiac output continuously during cardiac surgery are not yet reliable enough to be used as the input of a closed-loop system. The performance of the multiple-drug closed-loop system was evaluated intraoperatively in cardiac surgical patients who required simultaneous treatment with more than one vasoactive or inotropic drug.

\subsection{Material and method}

\subsubsection{Modelling drug responses}

The patient responses to changes in drug infusion rates were described by the transfer function of a first-order model with a transport delay. The parameters of the transfer function are illustrated by the model's response to a unit stepwise change in clrug infusion rate. The transport delay is the time before any change in pressure is visible and is equal to the time necessary for the drug to reach the target compartment. The half-life time of the drug describes the exponential change in pressure. The patient sensitivity is the final change in pressure after the effect of the stepwise change in drug infusion rate has stabilized. We obtained values for the first-order model parameters from our own experience with single-drug infusion control systems (chapters 7,8 and 9 ) and from previous publications on the drugs SNP (Sheppard 1980), NTG (Fahmy 1978), DOBU (Kates and Leier 1978) and NAD (Hoffman and Lefkowitz 1996). For the control of blood pressure, it is chiefly the variation in sensitivity between patients that is too large for a fixed control scheme and so necessitates an adaptive control strategy (Isaka and Sebald 1993). 


\subsubsection{Single-drug closed-loop control}

The design of a single-drug blood-pressure controller for the control of arterial hypertension using NTG was described in chapter 7. The single-drug controllers for the control of arterial hypotension, pulmonary hypertension and arterial hypertension using SNP were developed in a similar fashion. In short, a proportional-integral regulator calculated a new drug infusion rate every five seconds from the distance between the measured pressure and the target pressure and the integral of that distance. A supervisory computer program classified the patient into one of five sensitivity categories by observing the control performance of the proportional-integral regulator. The control gain of the proportional-integral regulator was inversely related to the patient sensitivity estimated online. Furthermore, the supervisory program defined the actions to be taken in cases of infusion pump problems, invalid pressure measurements and during unexpected periods of hypertension and hypotension.

The validity of the pressure signals was verified before calculating the mean pressure values. Each pressure beat was identified by its systolic upstroke and its shape was described by a set of eight characteristic features including mean, diastolic and systolic pressure and timing values (chapter 3). A pressure signal was considered invalid when the beat-to-beat fluctuations in the characteristic features were too large. Before validation, the pulmonary features were corrected for the ventilatory induced fluctuations by a least squares filtering technique (chapter 3 ). Mean arterial pressure (MAP) and mean pulmonary artery pressure (MPAP) were calculated every five seconds from the valid pressure beats and were considered valid when at least two valid pressure beats were found within a five-second episode.

\subsubsection{Multiple-drug control strategy}

The single-drug control strategy described above cannot be used for the simultaneous control of arterial and pulmonary pressures because of the difficulty of differentiating between drug effects when drug infusion rates are allowed to vary simultaneously. We decided to use a set of priority rules that intermittently activated one single-drug closed-loop controller out of a set of selected single-drug controllers to overcome this problem. The priority rules analysed the current drug infusion rates and the offset of MAP and MPAP from their target values. This strategy was based on our observation that an anaesthesiologist controls the most important haemodynamic variables first and tries to avoid simultaneous changes in 


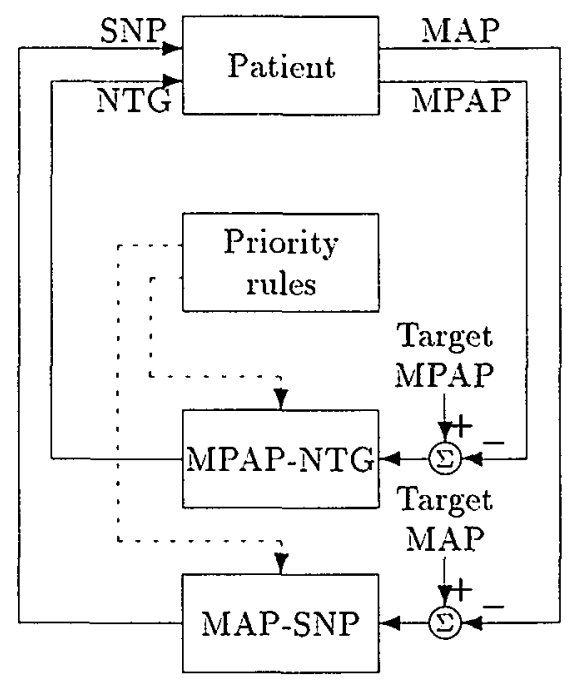

Figure 10.1: Scheme of the multiple-drug closed-loop system. In this example it is assumed that only the single-drug controllers MPAP-NTG and MAP-SNP have been selected by the anaesthesiologist. The priority rules automatically activate one of the selected controllers by analysing the control performance of the individual single-drug controllers and the patient's haemodynamic state. Dashed lines indicate the activation and deactivation actions of the priority rules. MAP, mean arterial pressure. MPAP, mean pulmonary artery pressure. NTG, nitroglycerin. SNP, sodium nitroprusside. (Not shown are the infusion pumps, the pressure validation routines and the non-selected single-drug controllers.) 
drug infusion rates. The control strategy is schematically shown in figure 10.1. The anaesthesiologist could select all possible combinations from the five single-drug controllers, with the exception of the combination MPAPNTG and MAP-NTG and the combination MAP-DOBU and MAP-NAD, neither of which could not be selected simultaneously.

\section{Expertise}

The initial knowledge that was used to construct the priority rules was obtained from standard anaesthetic protocols on perioperative management. of cardiac surgical patients (Kaplan and Guffin 1993) and from our own experience with single-drug blood-pressure control (chapters 7,8 and 9). Considerations that had to be taken into account when selecting a singledrug controller could be summarized as follows: arterial hypotension lowers perfusion pressures of vital organs, such as lidneys, heart and brain. Too high arterial pressures increase the workload of the left ventricle and may result in myocardial ischaemia, while high systolic pressures can damage fresh suture lines and increase intraoperative blood loss. Sudden increases in pulmonary pressure may indicate a situation of acute failing of leftventricular pump function. Too low pulmonary pressures may occur when circulatory filling is insufficient, but this situation is not controlled by the closed-loop system. The above criteria were further refined by discriminating between mild, moderate and severe hypertension and hypotension. The degree of hypertension was derived from the distances between the measured pressures and the target pressures. For a target MAP of $80 \mathrm{mmHg}$, the systemic arterial pressure could be normotensive $(75-85 \mathrm{mmHg})$, mildly hypertensive (85-95 $\mathrm{mmHg}$ ), moderately hypertensive $(95-100 \mathrm{mmHg})$, hypertensive $(100-120 \mathrm{mmHg}$ ) and severely hypertensive $(>120 \mathrm{mmHg})$. For a target MPAP of $20 \mathrm{mmHg}$, the pulmonary artery pressure could be normotensive $(17-23 \mathrm{mmHg})$, hypertensive $(23-27 \mathrm{mmHg})$ and severely hypertensive $(>27 \mathrm{mmHg})$. Comparable definitions held for arterial and pulmonary hypotension. Furthermore the priority rules take the characteristics of each drug into account. NTG was for example preferred to control mild arterial hypertension, but SNP was preferred to counter high arterial pressures because of its effectiveness in almost all patients.

\section{Implementation of expert knowledge}

A rule-based expert system was used to implement the priority rules for the activation of a single-drug controller. The expert system used two-valued logic ('true' or 'false') and kept track of the time that an evaluation had 
been true or false. The priority rules were implemented by an ordered list of if-then statements. The general construction of each if-then statement can be given as

if

a) 'no previous activation request found'

b) 'control of single-drug controller will be effective'

c) 'single-drug controller is selected by anaesthesiologist'

d) 'control of currently active single-drug controller is stable'

e) 'no conflicts with other single-drug controllers'

f) 'activation condition'

then

'activate single-drug controller'

Condition a) ensured that rules that were higher in the list had a higher priority. Control will be effective (condition b) when a drug infusion rate is not already limited at its minimum or maximum value. Condition c) ensured that only controllers that were selected by the anaesthesiologist could be activated. Activation rules with a lower priority required stability of the currently active controller (condition d), whereas rules with a higher priority required less or zero stability of the currently active controller. The stability of a single-drug controller was evaluated by analysing the slope of the associated drug infusion rate. Switching between controllers was not allowed during unexpectedly large changes in MAP. During an unexpectedly large increase of MAP the supervisory safety-shell kept all pressurelowering drug infusion rates constant and stopped all pressure-increasing drugs. Comparable actions were taken during an unexpectedly large decrease in MAP. Condition e) implemented some logical constraints that prevented, for example, the MAP-NAD controller from being activated as long as the SNP drug infusion rate was non-zero. The activation condition (condition $\mathrm{f}$ ) held the actual condition that triggered the activation of the single-drug controller.

The order of the if-then rules in the rule base determined the priority that was given to each rule (because of condition a). This is illustrated by the following simplified example, where only the activation conditions (condition $f$ ) are given

if 'severe pulmonary hypertension' then 'activate MPAP-NTG'

if 'arterial hypertension' then 'activate MAP-SNP'

if 'pulmonary hypertension' then 'activate MPAP-NTG'

In this example severe pulmonary hypertension is controlled first, but control of arterial hypertension has a higher priority than control of pulmonary hypertension. Table 10.1 summarizes all the rules that were used to acti- 
vate a different single-drug controller during the clinical evaluation. During drug-induced hypotensive and hypertension, high priority was given to the lowering of drug infusion rates. Next, the various combinations of arterial and pulmonary hypertension and hypotension were dealt with. Rules with a lower priority tried to deactivate a single-drug controller when its control had proved ineffective. Control was ineffective when the pressure remained constant for some time after the drug infusion rate had changed or when the drug infusion rate was limited at its maximal or minimal value. The rules with the lowest priority tried to activate a different controller every six minutes, provided the currently active controller had achieved its control goal.

All single-drug blood-pressure controllers, the priority rules and the supervisory safety-shell were programmed in Pascal (Turbo Pascal v7.0, Borland). The user interface comprised five trend displays of MAP, MPAP and the infusion rates of NTG, SNP, and NAD or DOBU. Numeric boxes displayed current infusion rates, heart-rate and mean, systolic and diastolic arterial and pulmonary pressure values. Function keys were used to select or deselect blood-pressure controllers and to change target pressures and infusion rates of non-selected controllers. An emergency stop-key enabled the anaesthesiologist to shut down all infusions in the unlikely event of a system failure.

\subsubsection{Clinical evaluation}

\section{Patients}

The multiple-drug infusion system was used during cardiac surgery in 30 patients who required simultaneous treatment with more than one of the drugs SNP, NTG, NAD or DOBU. Most patients were male (20 of the 30 ). Patients' weights varied between 42 and $100 \mathrm{~kg}$ (average $72.9 \mathrm{~kg}$ ). Patients' ages varied between 41 and 77 years (average 65.6 years). Patients were scheduled either for coronary bypass surgery (18 cases), valvular surgery (10 cases) or other (two cases). The study was approved by our hospital's medical ethics committee. Informed written patient consent was obtained the evening before surgery.

\section{Anaesthesia}

All patients received lorazepam as oral premedication two hours before surgery. Anaesthesia was induced with $7.5 \mu \mathrm{g} \mathrm{kg}-1$ sufentanil and maintained with $3.75 \mu \mathrm{g} \mathrm{kg}^{-1} \mathrm{~h}^{-1}$ sufentanil. Muscle relaxation was achieved 
with $0.1 \mathrm{mg} \mathrm{kg}^{-1}$ of pancuronium bromide. The lungs were ventilated with an oxygen/air mixture $\left(\mathrm{F}_{\mathrm{i}} \mathrm{O}_{2}=0.50\right)$ at a rate of 15 breaths min ${ }^{-1}$ and ventilatory volume was adjusted to maintain arterial $\mathrm{CO}_{2}$ tension between 32 and $42 \mathrm{mmHg}$. The pulmonary artery pressure was measured from the distal port of a pulmonary artery catheter ( $7.5 \mathrm{~F}$, Baxter) which was introduced prior to surgery via the right internal jugular vein. The systemic arterial pressure signal was measured using a saline-filled radial artery catheter (20-gange, Arrow). Pressure transducers (Uniflow, Baxter) were used to convert the pressure signals to an electrical signal. Pressure signals were taken from the operating-room monitoring system (M1168A Components Monitoring System, Hewlet. Packard) and digitized at a sampling rate of 100 Hertz using a 12-bits analog-to-digital converter (DAS1600, Keithley Metrabyte). Low concentrations of all drugs were used to prevent excessive dosages in the unlikely event of system malfunction and to minimize effective dead space in the infusion lines. SNP and NTG were both diluted to $200 \mu \mathrm{g} \mathrm{ml}^{-1}$, DOBU to $1 \mathrm{mg} \mathrm{ml}^{-1}$ and NAD to $5 \mu \mathrm{g} \mathrm{ml} \mathrm{m}^{-1}$. Drugs were infused via the introduction sheet of the pulmonary artery catheter by computer-controlled syringe infusion pumps (P4000, IVAC corporation) using a RS232 communication protocol. A software handshake protocol ensured safe communication between computer and infusion pumps. A continuous infusion of $150 \mathrm{ml} \mathrm{hr}^{-1}$ sodium chloride was added to minimize dead time in the infusion lines.

After cardiopulmonary bypass, patients who underwent surgery for coronary bypass grafting received, independent of the study program, a separate infusion of $0.5 \mu \mathrm{g} \mathrm{kg}^{-1} \mathrm{~min}^{-1}$ NTG to prevent spasm of mammary and coronary arteries. During the procedure an intravenous bolus of 5 $\mathrm{mg}$ alfentanil was given when an insufficient depth of anaesthesia was suspected. Manually controlled DOBU was used in normotensive patients with low cardiac output.

Cardiac output measurements were performed at the start of the operation, before and after cardiopulmonary bypass and at the end of the operation. When required additional cardiac output values were measured during the operation at haemodynamically stable intervals. Cardiac output was calculated from four thermodilution measurements taken at equidistantly spaced intervals over the ventilator cycle (Jansen et al. 1990a). Between thermodilution measurements, cardiac output was calculated from the arterial pressure waveform using a modified pulse contour method (Wesseling et al. 1993). All cardiac output measurements were indexed to the patient's body surface area. 


\section{Usage during operation}

At the start of the operation the anaesthesiologist chose the target pressures for MAP and MPAP and selected those blood pressure controllers that were allowed to be activated by the priority rules. Generally, MAPSNP was selected in patients with systemic hypertension and MAP-NTG in ischaemic hypertensive patients. MPAP-NTG was selected to control elevated pulmonary pressures secondary to left ventricular heart failure or mitral valve defects. MAP-DOBU was used to control low arterial pressures secondary to low output failure. MAP-NAD was used to control low arterial pressures in the presence of high cardiac output resulting from an anaphylactic or cardiogenic shock. The multiple-drug closed-loop system was used to control pressures from the start of the operation up to the start of the cardiopulmonary bypass and from the end of the cardiopulmonary bypass until the end of the operation.

\subsection{Results}

The duration of the operation, before and after cardiopulmonary bypass was respectively $39.9 \pm 19.9$ (mean $\pm \mathrm{SD}$ ) and $45.3 \pm 13.3$ minutes. Illustrative examples of the controller's performance during surgery are given in figures $10.2,10.3$ and 10.4 . Figure 10.5 shows the combinations of single-drug controllers that were selected by the anaesthesiologist. Table 10.2 show:s the percentages of time that a single-drug controller was active. Table 10.1 shows the priority rules that were used by the closed-loop system to select a different single-drug controller. The anaesthesiologist on no occasion overruled the controller's actions manually. None of the single-drug closedloop systems showed any sign of unstable behaviour.

Before cardiopulmonary bypass, the average computer-controlled infusion rates of NTG, SNP, DOBU and NAD were respectively $1.04 \pm 1.15$, $0.69 \pm 0.61,3.63 \pm 2.00$ and $18.9 \cdot 10^{-3} \pm 18.1 \cdot 10^{-3} \mu_{\mathrm{g} \mathrm{kg}}^{-1} \mathrm{~min}^{-1}$. After cardiopulmonary bypass, the average computer-controlled infusion rates of NTG, SNP, DOBU and NAD were respectively $0.66 \pm 0.7 \tau, 0.41 \pm 0.34$, $5.12 \pm 4.05$ and $35.4 \cdot 10^{-3} \pm 30.9 \cdot 10^{-3} \mu \mathrm{g} \mathrm{kg} \mathrm{kg}^{-1} \mathrm{~min}^{-1}$. All zero infusion rates and the $0.5 \mu \mathrm{g} \mathrm{kg}^{-1} \mathrm{~min}^{-1}$ NTG infusion after cardiopulmonary bypass in patients awaiting coronary bypass grafting were excluded when calculating the average infusion rates. The average target values of MAP and MPAP were respectively $79.9 \pm 5.6$ and $20.7 \pm 1.6 \mathrm{mmHg}$ before cardiopulmonary bypass and $77.7 \pm 5.5$ and $20.6 \pm 1.7 \mathrm{mmHg}$ after cardiopul- 


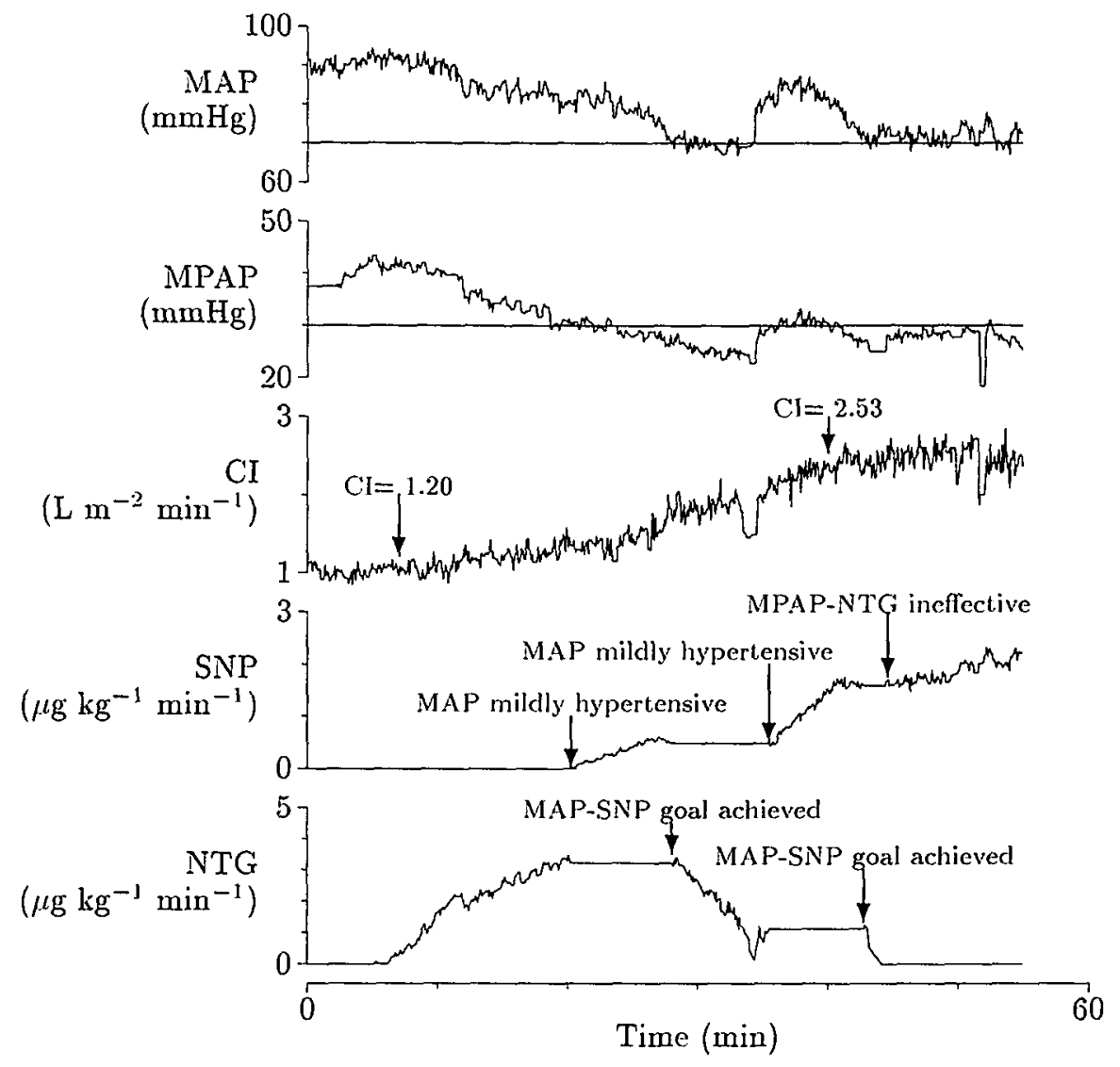

Figure 10.2: Recordings before cardiopulmonary bypass of a 52-year-old male patient with end-stage heart failure and a left ventricular ejection fraction of 12 $\%$. The anaesthesiologist selected the MPAP-NTG controller at the start of the operation and the MAP-SNP controller at 20 min. The priority rules that were used by the closed-loop system to automatically activate a different controller and the calibrations of the cardiac index $(\mathrm{CI})$ by thermodilution measurements are indicated inside the figure. MAP, mean arterial pressure. MPAP, mean pulmonary artery pressure. NTG, nitroglycerin. SNP, sodium nitroprusside. 


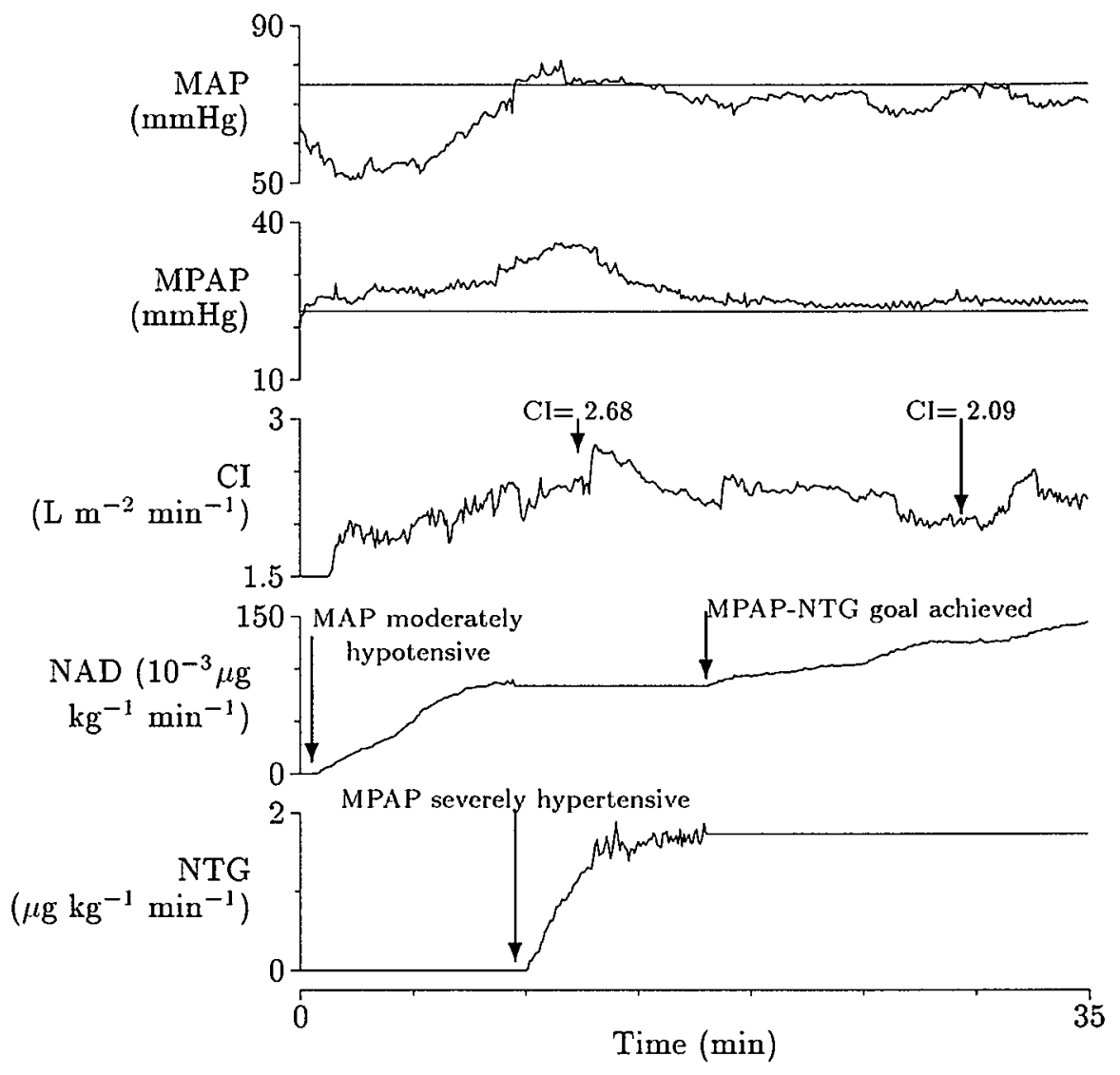

Figure 10.3: Recordings of a 73-year-old female patient. Measurements start when the patient is still on cardiopulmonary bypass. The MAP-NAD, MAP-SNP and MPAP-NTG controllers were selected by the anaesthesiologist at $0 \mathrm{~min}$. Cardiopulmonary bypass is stopped at $2 \mathrm{~min}$. The priority rules that were used by the closed-loop system to automatically activate a different controller and the calibrations of the cardiac index (CI) by thermodilution measurements are indicated inside the figure. At 30 min a continuous manual infusion of $3 \mu \mathrm{g} \mathrm{kg}^{-1} \mathrm{~min}^{-1}$ DOBU was started. MAP, mean arterial pressure. MPAP, mean pulmonary artery pressure. NAD, noradrenalin. NTG, nitroglycerin. 


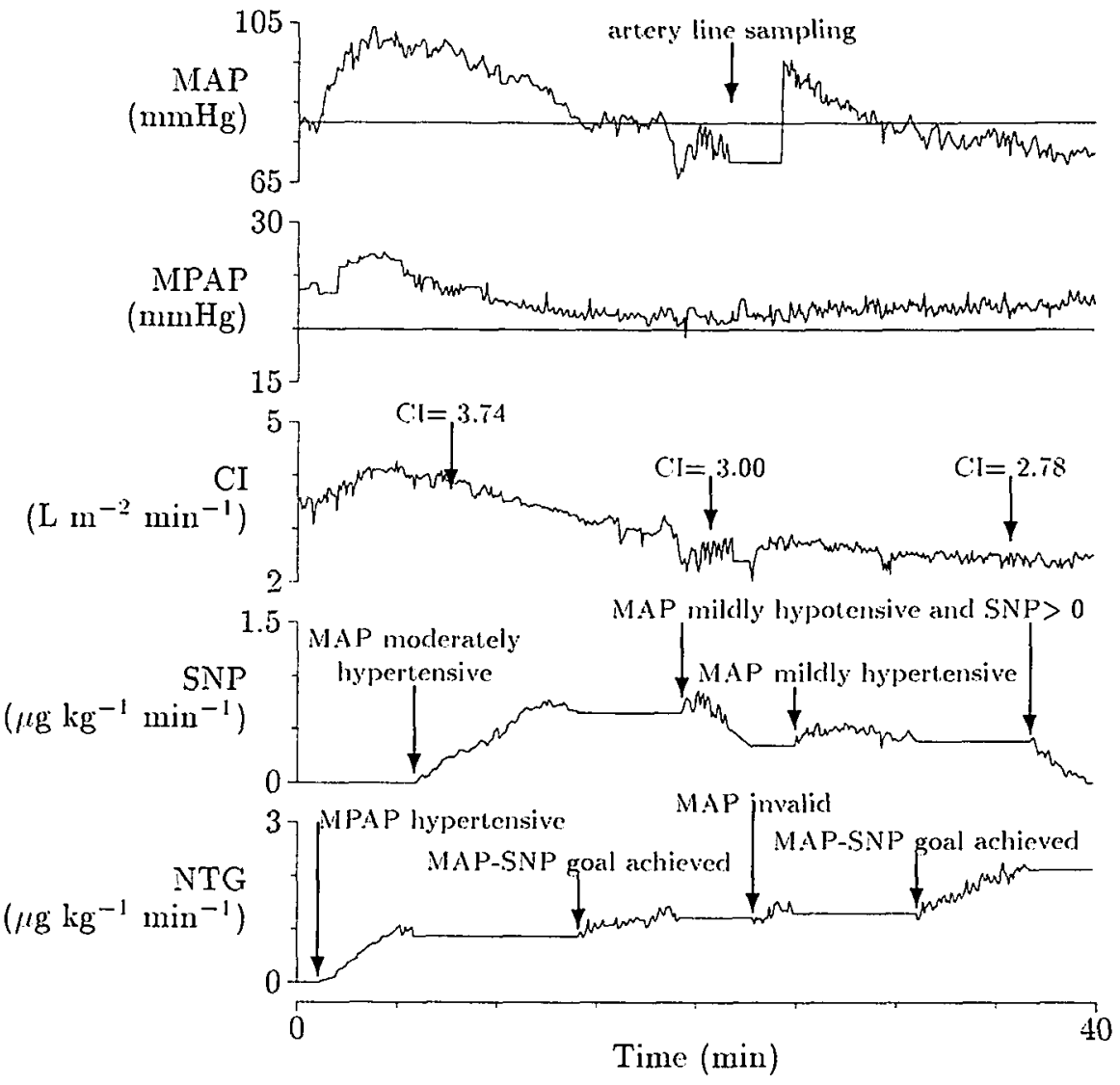

Figure 10.4: Recordings after cardiopulmonary bypass of a 46-year-old male patient with a left ventricular ejection fraction of $20 \%$. The MAP-SNP and MPAP-NTG controllers were selected by the anaesthesiologist at 0 min. The priority rules that were used by the closed-loop system to automatically activate a different controller and the calibrations of the cardiac index (CI) by thermodilution measurements are indicated inside the figure. MAP, mean arterial pressure. MPAP, mean pulmonary artery pressure. NTG. nitroglycerin. SNP, sodium nitroprusside. 


\begin{tabular}{llll}
\hline$\#$ & Controller & Priority rule & Action \\
\hline 1 & MAP-DOBU & MAP mildly hypertensive and DOBU >0 & DOBU $\downarrow$ \\
4 & MAP-NAD & MAP mildly hypertensive and NAD >0 & NAD $\downarrow$ \\
0 & MAP-SNP & MAP hypotensive and SNP >0 & SNP $\downarrow$ \\
0 & MAP-NTG & MAP hypotensive and NTG $>0$ & NTG $\downarrow$ \\
1 & MAP-SNP & MAP severely hypertensive & SNP $\uparrow$ \\
1 & MPAP-NTG & MPAP severely hypotensive and NTG >0 & NTG $\downarrow$ \\
16 & MAP-SNP & MAP mildly hypotensive and SNP >0 & SNP $\downarrow$ \\
2 & MAP-NTG & MAP mildly hypotensive and NTG >0 & NTG $\downarrow$ \\
13 & MPAP-NTG & MPAP hypotensive and NTG $>0$ & NTG $\downarrow$ \\
4 & MAP-DOBU & MAP hypotensive & DOBU $\uparrow$ \\
4 & MAP-NAD & MAP hypotensive & NAD $\uparrow$ \\
13 & MPAP-NTG & MPAP severely hypertensive & NTG $\uparrow$ \\
5 & MAP-DOBU & MAP moderately hypotensive & DOBU $\uparrow$ \\
6 & MAP-NAD & MAP moderately hypotensive & NAD $\uparrow$ \\
15 & MAP-SNP & MAP hypertensive & SNP $\uparrow$ \\
2 & MAP-NTG & MAP moderately hypertensive & NTG $\uparrow$ \\
2 & MAP-SNP & MAP moderately hypertensive & SNP $\uparrow$ \\
37 & MPAP-NTG & MPAP hypertensive & NTG $\uparrow$ \\
11 & MAP-NTG & MAP mildly hypertensive & NTG $\uparrow$ \\
46 & MAP-SNP & MAP mildly hypertensive & SNP $\uparrow$ \\
32 & MAP-DOBU & MAP mildly hypotensive & DOBU $\uparrow$ \\
16 & MAP-NAD & MAP mildly hypotensive & NAD $\uparrow$ \\
73 & all & ineffective control & - \\
52 & all & control goal achieved & - \\
\hline
\end{tabular}

Table 10.1: The number of times that a priority rule was used to activate a different blood-pressure controller (indicated by \#). Rules at the top of the list had the highest priority. Data of all 30 patient were analysed. All rules that fired when control of the currently active controller was ineffective or when the currently active controller had reached its control goal were grouped (all). Total number of activations was 356 . On average one activation every 7.2 min. See text for further explanation. 


\begin{tabular}{lccc}
\hline Controller & Selected & Active & Ratio \\
\hline MAP-SNP & 91 & 46 & 51 \\
MAP-NTG & 15 & 9 & 60 \\
MPAP-NTG & 67 & 22 & 33 \\
MAP-NAD & 20 & 8 & 40 \\
MAP-DOBU & 38 & 15 & 39 \\
\hline
\end{tabular}

Table 10.2: The percentages of time that a single-drug controller was selected by the anaesthesiologist, activated by the rule base and the ratio of these percentages expressed in percentages.

monary bypass. The average deviations from the target MAP and MPAP were respectively $8.6 \pm 4.0$ and $4.4 \pm 4.0 \mathrm{mmHg}$ before cardiopulmonary bypass and $8.0 \pm 3.6$ and $2.4 \pm 0.9 \mathrm{mmHg}$ after cardiopulmonary bypass. The cardiac index before the start of the operation, before and after the cardiopulmonary bypass and at the end of the operation was $2.46 \pm 0.52$, $2.83 \pm 0.75,2.93 \pm 0.55$ and $2.72 \pm 0.62 \mathrm{~L} \mathrm{~min}^{-1}$, respectively.

Computer control of NTG was used in 26 patients, of SNP in 27 patients, of NAD in 10 patients and of DOBU in nine patients. In three patients a low-dose dopamine infusion $\left(2 \mu \mathrm{g} \mathrm{kg}^{-1} \mathrm{~min}^{-1}\right)$ was given after cardiopulmonary bypass for kidney function. In three patients a manually controlled infusion of DOBU was used because of low cardiac output at non-hypotensive arterial pressure levels. In one patient arterial pressure could not satisfactorily be increased after cardiopulmonary bypass using DOBU. In another patient both NAD and DOBU could not satisfactorily increase arterial pressure after cardiopulmonary bypass.

\subsection{Discussion}

A sufficient depth of anaesthesia and adequate circulatory filling are needed in order to control haemodynamics successfully. We ensured adequate circulatory filling by manually infusing additional volume when MPAP fell below 18-20 $\mathrm{mmHg}$. MPAP was used as an indicator of the pulmonary wedge pressure, which is a valid assumption in the absence of pulmonary problems. To guarantee adequate anaesthetic levels, a continuous infusion of opioids was used. Additional bolus infusions of alfentanil were given during the procedure when an insufficient depth of anaesthesia was suspected.

Incorrect setup of infusion lines can quickly instigate a delay of several minutes, which might trigger oscillatory behaviour of the control system. We minimized the dead time in the drug infusion lines by using large vol- 


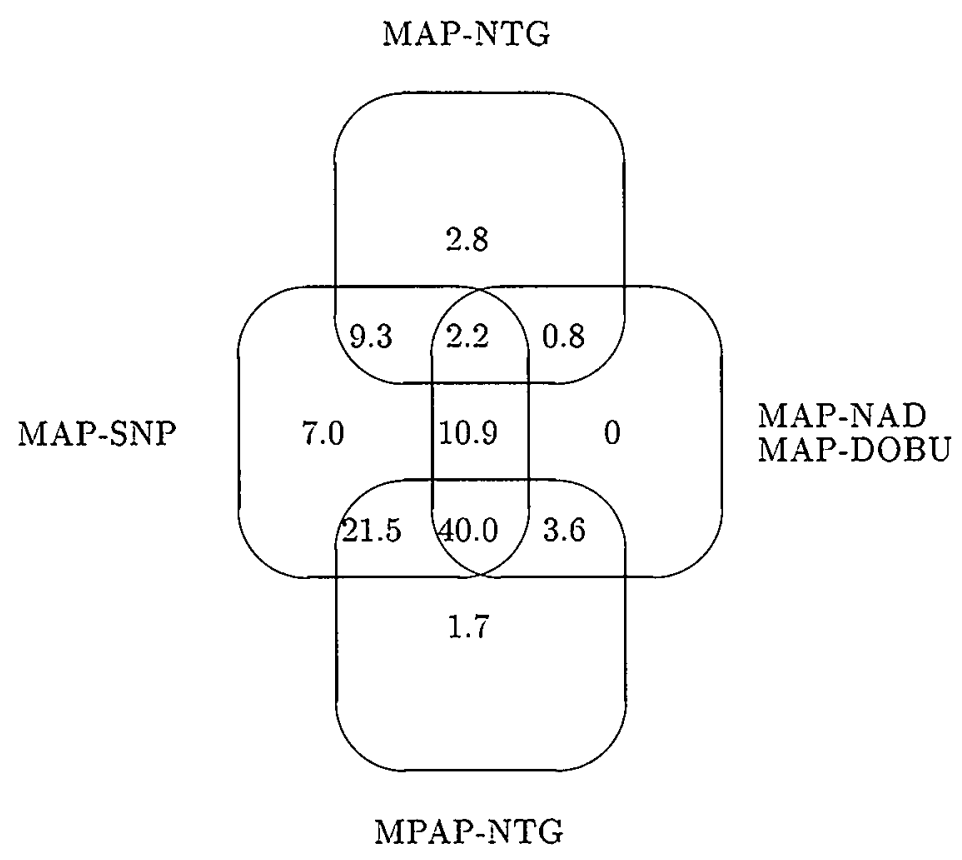

Figure 10.5: All possible combinations of the single-drug controllers. Numbers indicate the percentages of time that the different single-drug controller combinations were selected by the anaesthesiologist. The results of the MAP-NAD and MAP-DOBU controllers have been combined. 
umes of low drug concentrations, purging all lines at start-up, using short (direct) infusion lines and by adding a sufficient continuous infusion to the shared infusion line. Since oscillatory behaviour was never observed, we conclude that our efforts to minimize dead-time were adequate.

Closed-loop control of blood pressure requires online identification of the patient model parameter values to achieve optimum tuning of the controller for a given patient (Isaka and Sebald 1993). In a clinical setting online identification of the patient model is hampered by high background noise, surgical manipulations, infusion of other drugs and drifting of zerodrug-infusion pressure owing to compensatory reflexes and changes in the patient's haemodynamic condition. We therefore limited the online identification of the patient model parameters for each drug to the classification of the patient into one of five sensitivity categories. The classification of the patient sensitivity was automatically updated when the progress of the pressure towards the target pressure was either too fast or too slow. In addition we used a supervisory computer program, which enabled the system to exercise simulated common sense under all circumstances. The effectiveness of this control strategy has been demonstrated previously for the control of arterial hypertension using NTG (chapter 7). Results of the present study demonstrated that the same control strategy can also be used to control other haemodynamic states, such as pulmonary hypertension and arterial hypotension.

For the control of arterial hypotension in patients with a low cardiac output we preferred use of DOBU over the inotropic drug dopamine, because of the nonlinear behaviour of dopamine at lower infusion rates (Held and Roy 1995). NAD was used to control arterial hypotension with high cardiac output secondary to a cardiogenic or anaphylactic shock. Because the choice of anti-hypotensive drug depends strongly on the level of the cardiac output, cardiac output should be closely monitored when controlling arterial hypotension. To monitor changes in cardiac output we used a continuous cardiac output monitoring system (Wesseling et al. 1993), which was calibrated by thermodilution measurements taken at equidistantly spread intervals over the ventilator cycle (Jansen et al. 1990a).

Voss et al. (1987) and Yu et al. (1992) both described multiple-drug closed-loop systems that control MAP and cardiac output by simultaneously varying the infusion rates of a vasodilator and an inotropic drug. Both systems were evaluated in dogs and an aortic flow probe was used to measure cardiac output continuously. In a clinical setting, however, online estimates of the patient model are not reliable when drug infusion rates are allowed to vary simultaneously, because of the difficulty of differentia- 
tion between drug effects. We avoided this problem by allowing only one drug to be varied at one time. This strategy coincides with the normal working procedure of the anaesthesiologist, who tries to stabilize the most critical haemodynamic variables first and avoids simultaneous changes in drug infusion rates. The anaesthesiologist could still determine the basic drug-regime by selecting target pressures and single-drug controllers that were allowed to be activated by the priority rules, whereas the routinelike adjustments of drug infusion rates were taken over by the closed-loop system. Decisions concerning the basic drug-regime were not automated, because of the superiority of the anaesthesiologist's background information insight into the patient condition. Elevated pulmonary pressures might for example indicate an acute failing of left-ventricular pump function, but they might also indicate hypoxia.

By intermittently controlling drug infusion rates, the multiple-drug closed-loop system satisfactorily controlled both arterial and pulmonary pressures during cardiac surgery. The selection of single-drug controllers by the priority rules was sufficiently fast and control of each single-drug controller was stable. The ultimate goal of a multiple-drug infusion system is to control arterial and pulmonary artery pressures and cardiac output simultaneously, in such a way that an adequate cardiac output can be obtained at a normalized preload and afterload for a given patient. As soon as a reliable continuous cardiac output measurement technique is available, a single-drug controller for the control of cardiac output might be developed and integrated into the multiple-drug closed-loop system simply by adding priority rules. Two additional advantages of the proposed technique of using priority rules are that it involves the clinician more in the development of the control system and that it is intuitively appealing for a clinician. Both factors will facilitate the acceptance of this new technique in the clinical environment. We expect that closed-loop control of multiple drugs can further extend the improvements in patient outcome, that were found by Chitwood et al. (1992) in postoperative hypertensive patients, to the perioperative haemodynamically unstable patient. Future research, in a large patient population and preferably in a multi-centre trial setting; should therefore concentrate on the clinical relevance of the multiple-drug closed-loop system in terms of improved patient outcome. 


\title{
Chapter 11
}

\section{Computer control versus manual control of systemic hypertension during cardiac surgery}

\author{
S. A. A. P. Hoeksel \\ J. A. Blom \\ J. R. C. Jansen \\ J. G. Maessen \\ J. J. Schreuder
}




\begin{abstract}
Objective. To investigate whether computer control of vasodilator drug infusion rates improves haemodynamic stability in patients undergoing cardiac surgery.

Material and method. Systemic arterial blood pressures were managed either by computer ( 80 patients) or by a well-trained anaesthesiologist ( 80 patients). The vasodilator drugs sodium nitroprusside and nitroglycerin were used. Haemodynamic stability was determined from the standard deviation of the mean arterial pressure samples and from the percentages of time that arterial pressure was hypertensive or hypotensive.

Results. The average standard deviation of the mean arterial pressure was smaller in the computer samples than for the manually controlled group; $7.5 \pm 2.2$ (mean $\pm \mathrm{SD}$ ) versus $8.9 \pm 2.3 \mathrm{mmHg}(p<0.0001)$. The systemic arterial pressure was less hypertensive and less hypotensive in the computer controlled than in the manually controlled group; respectively $9.4 \pm 5.7$ versus $13.1 \pm 6.0 \%(p<0.0001)$ and $8.0 \pm 5.9$ versus $11.8 \pm 7.4 \%$ $(p<0.0001)$.

Conclusions. Compared with manual control, computer control of systemic hypertension significantly improved haemodynamic stability during cardiac surgery.
\end{abstract}

Keywords cardiac surgery $\bullet$ hypertension $\bullet$ closed-loop control $\bullet$ outcome study 


\subsection{Introduction}

Several investigators have demonstrated that computer control of vasodilator infusion rates for the management of postoperative systemic hypertension in cardiac surgical patients results in more stable haemodynamic profiles when compared with manual control (de Asla et al. 1985; Reid and Kenny 1987; Colvin and Kenny 1989; Cosgrove III et al. 1989). Chitwood et al. (1992) demonstrated in a multi-centre trial that more stable haemodynamic profiles, obtained by computer control, improve outcome for the postoperative cardiac surgical patient. Compared with manual control, computer control decreases the incidence of therapy-induced hypotension, reduces postoperative bleeding, decreases transfusion requirements and probably decreases the incidence of re-exploration for postoperative bleeding (Chitwood et al. 1992).

Control of systemic hypertension during cardiac surgery is more challenging than control of systemic hypertension in the intensive care unit, because in the operating room a faster control is required and conditions might change more rapidly (Martin et al. 1992b). Results of preliminary investigations into the feasibility of computer control of systemic hypertension during cardiac surgery have been described independently by Blom (1991), Martin et al. (1992b) and Meijers et al. (1997), whereas only the recent study by Meijers et al. (1997) claimed an acceptable control performance in all patients. Blom (1991) also compared the performance of computer control with manual control and found that in the computer controlled group MAP was on average closer to the target MAP, though no significance levels were given. Moreover, haemodynamic stability was expressed as the variation around a predefined target pressure, a measure that might favour computer control, because clinicians, unlike closed-loop systems, do not work with predefined target pressures.

We described a strategy for the control of systemic hypertension during cardiac surgery using the vasodilators sodium nitroprusside (SNP) and nitroglycerin (NTG) in chapters 7 and 8 . The aim of the present study was to investigate whether use the of these computerized infusion systems improves haemodynamic stability of patients undergoing cardiac surgery. 


\subsection{Material and method}

\subsubsection{Patients and anaesthesia}

The study was approved by our hospital's medical ethics committee. Patients gave informed consent for use of their data in this study on the evening before surgery. All cardiac surgical patients that were treated by the same anaesthesiologist over a period of three years were included in the study protocol. Patients who developed systemic hypertension during cardiac surgery and required treatment with vasodilator drugs were analysed. The type of control used depended on the availability of the measuring equipment and of a control engineer, who analysed the way the computerized system was used by the clinicians. Retrospective data were provided by the manually controlled group. There were 80 patients in the computercontrolled group, and 80 patients in the manually controlled group. Patients were scheduled either for coronary artery bypass surgery (139 cases) or valvular surgery (21 cases), aged $63.0 \pm 9.4$ (mean \pm SD) years and weighed $78.7 \pm 12.2 \mathrm{~kg}$. Most patients were male (120 out of 160 ). No statistically significant differences could be detected between patient groups relating to type of surgery and patient gender, weight or age.

All patients received comparable anaesthetic regimes; starting with lorazepam as oral premedication two hours before surgery. Patients scheduled for coronary bypass surgery received triple medication according to a general protocol inclucling nitrates, $\beta$-blockades and Ca-antagonists. Anaesthesia was induced with $7.5 \mu \mathrm{g} \mathrm{kg}^{-1}$ sufentanil and maintained with $3.75 \mu \mathrm{g}$ $\mathrm{kg}^{-1} \mathrm{~h}^{-1}$ sufentanil. Patients who underwent coronary bypass surgery received, independently of the study programme, a baseline infusion of NTG $\left(0.5 \mu \mathrm{g} \mathrm{kg}^{-1} \mathrm{~min}^{-1}\right)$ after cardiopulmonary bypass to prevent spasm of coronary and mammary arteries. SNP and NTG infusions were prepared in a similar fashion for both patient groups and infused by syringe infusion pumps; for the computer controlled group an IVAC P4000 (IVAC) and for the manually controlled group a Treonic IP4 (Vickers) infusion pump. A $7 \mathrm{~F}$ pulmonary artery catheter was introduced prior to surgery via the right internal jugular vein. The systemic arterial pressure signal was obtained using a saline-filled radial artery catheter (20 gauge).

In the manually controlled patient group systemic blood pressures were controlled manually by the anaesthesiologist. In the computer-controlled patient group either the SNP or the NTG infusion rate was controlled by computer. The design of the closed-loop blood-pressure controllers has been described elsewhere (chapters 7 and 8 ). In both groups the choice of 
vasodilator was made by the anaesthesiologist. In the computer controlled group, the vasodilator drug that was controlled by the closed-loop system could be changed at any time by the anaesthesiologist. During cardiopulmonary bypass vasodilator infusion rates were controlled manually in both groups.

\subsubsection{Measurements}

The arterial pressure signal was taken from the pressure module (M1006A, Hewlett Packard) of the operating room monitoring system (M1168A component monitoring system, Hewlett Packard). In both patient groups, the arterial pressure waveform was analysed online by the ModelFlow ${ }^{\mathrm{TM}}$ cardiac output monitor system (Wesseling et al. 1993). The ModelFlow ${ }^{\text {TM }}$ system stored several features of each pressure beat on the hard disk of an IBM-AT compatible personal computer and provided a trend recording of the arterial pressure during the operation. Moreover, it allowed us to analyse the pressure data independently of the type of control used. The validity of each arterial pressure beat was determined offline by analysing the beat-to-beat fluctuations in its characteristic features (chapter 3). MAP was calculated every five seconds from the valid pressure beats. As stability indices we used the standard deviation of the 5 -second averaged MAP samples and the percentages of time that MAP was $10 \mathrm{mmHg}$ above and below the average MAP. For each patient the stability indices before and after cardiopulmonary bypass were averaged.

During a haemodynamically stable interval arterial pressures and cardiac output measurements were done before the start of the operation, before the start of the cardiopulmonary bypass, after the cardiopulmonary bypass and at the end of the operation. For these measurements, pressure and heart-rate measurements were averaged over 30 seconds to reduce the influence of small fluctuations. Cardiac output was calculated from a set of four thermodilution measurements taken at equally spaced distances over the ventilator cycle (Jansen et al. 1990a). Cardiac output measurements were indexed to patient body surface area. Additional haemodynamic measurements were taken during the procedure when required.

The stability indices were analysed statistically using regression analysis. As factors for the regression model the following variables were used; MAP and cardiac index measurements taken before the start of operation, date of the operation, mean SNP and NTG infusion rates, type of surgery, duration of surgery, type of control and patient age and gender. Dummy variables were used to code patient gender, type of control and type of 


\begin{tabular}{|c|c|c|c|c|}
\hline & Start-Oper & Start-Bypass & End-Bypass & End-Oper \\
\hline & \multicolumn{4}{|c|}{ Computer controlled } \\
\hline MAP (mmHg) & $83.0 \pm 13.7$ & $79.3 \pm 7.7$ & $72.0 \pm 9.9$ & $76.0 \pm 7.4$ \\
\hline$P_{\text {art }} \mathrm{s}(\mathrm{mmHg})$ & $123.6 \pm 20.0$ & $124.2 \pm 14.6$ & $108.0 \pm 16.1$ & $114.1 \pm 11.4$ \\
\hline$P_{\text {art }} \mathrm{d}(\mathrm{mmHg})$ & $62.9 \pm 11.6$ & $60.5 \pm 8.1$ & $55.2 \pm 7.8$ & $60.2 \pm 6.9$ \\
\hline CI $\left(\mathrm{L} \min ^{-1} \mathrm{~m}^{2}\right)$ & $2.3 \pm 0.6$ & $2.6 \pm 0.7$ & $2.9 \pm 0.7$ & $2.6 \pm 0.7$ \\
\hline \multirow[t]{2}{*}{$\mathrm{HR}$ (beats $\min ^{-1}$ ) } & $63.1 \pm 13.5$ & $74.4 \pm 19.8$ & $79.5 \pm 12.2$ & $83.5 \pm 12.4$ \\
\hline & \multicolumn{4}{|c|}{ Manually controlled } \\
\hline $\operatorname{MAP}(\mathrm{mmHg})$ & $84.0 \pm 11.0$ & $80.0 \pm 10.2$ & $72.8 \pm 9.8$ & $80.0 \pm 10.7$ \\
\hline $\mathrm{P}_{\text {art }} \mathrm{s}(\mathrm{mmHg})$ & $126.9 \pm 16.9$ & $126.2 \pm 19.1$ & $110.6 \pm 17.6$ & $121.5 \pm 17.5$ \\
\hline$P_{\text {art }} \mathrm{d}(\mathrm{mmHg})$ & $63.4 \pm 9.3$ & $62.2 \pm 8.9$ & $56.9 \pm 8.0$ & $64.2 \pm 9.0$ \\
\hline $\mathrm{CI}\left(\mathrm{L} \min ^{-1} \mathrm{~m}^{2}\right)$ & $2.4 \pm 0.5$ & $2.6 \pm 0.5$ & $3.1 \pm 0.7$ & $2.7 \pm 0.5$ \\
\hline $\mathrm{HR}$ (beats $\min ^{-1}$ ) & $63.6 \pm 12.5$ & $74.6 \pm 17.8$ & $83.2 \pm 14.9$ & $86.6 \pm 15.1$ \\
\hline
\end{tabular}

Table 11.1: Haemodynamic data of computer- and manually controlled patient groups. Measurements were taken before the start of the operation (Start-Oper), before (Start-Bypass) and after cardiopulmonary bypass (End-Bypass) and at the end of the operation (End-Oper). Values are mean \pm SD. MAP, mean arterial pressure. $P_{a r t} s$, systolic arterial pressure. $P_{\text {art }} \mathrm{d}$, diastolic arterial pressure. $\mathrm{CI}$, cardiac output indexed to body surface area. HR, heart rate.

surgery. Differences between patient groups were analysed by an unpaired Student's $t$ statistic. A non-parametric Mann-Witney statistic was used to analyse dichotomous data. A significance level of $p<0.05$ was considered to be significant in all cases.

\section{$11.3 \quad$ Results}

The duration of the operation before and after cardiopulmonary bypass was respectively $61.4 \pm 28.7$ and $49.0 \pm 23.7$ minutes for the computer and $62.7 \pm 28.4$ and $49.4 \pm 18.5$ minutes for the manually controlled group. SNP was used more often in the computer- than in the manually controlled group; 55 out of 80 versus 35 out of 80 patients $(p<0.01)$. NTG was used less frequently in the computer-controlled group; 49 out of 80 versus 66 out of 80 patients $(p<0.01)$. Average SNP infusion rates were higher in the computer- than in the manually controlled group; $0.62 \pm 0.49$ versus $0.35 \pm 0.34 \mu \mathrm{g} \mathrm{kg}^{-1} \min ^{-1}(p<0.01)$. Average NTG infusion rates in the computer and manually controlled group were respectively $0.52 \pm 0.41$ and $0.55 \pm 0.32 \mu \mathrm{g} \mathrm{kg}^{-1} \mathrm{~min}^{-1}$. Zero-infusion rates (from patients who did not require one of the vasodilator drugs) were ignored when the average infusion rate for that vasodilator was calculated. Average MAP before and after cardiopulmonary bypass was respectively $82.0 \pm 6.1$ and $74.7 \pm 6.6 \mathrm{mmHg}$ 

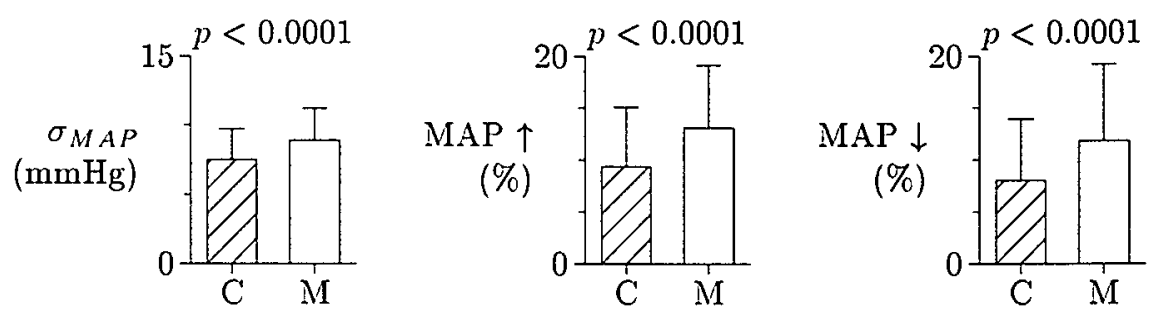

Figure 11.1: Results of computer control (C) versus manual control (M) of mean arterial pressure (MAP). Shown are the standard deviation of MAP samples $\left(\sigma_{M A P}\right)$ and the percentages of time that MAP was hypertensive (MAP $\left.\uparrow\right)$ and hypotensive (MAP $\downarrow$ ). Shown are mean+SD of all patients.

for the computer-controlled group and $83.7 \pm 7.0$ and $76.5 \pm 7.9 \mathrm{mmHg}$ for the manually controlled group. There were no statistically significant differences between groups in duration of operation, mean NTG infusion rate and average MAP levels before and after cardiopulmonary bypass.

No significant differences between the patient groups could be detected in haemodynamic measurements before the start of the operation, before the cardiopulmonary bypass and after cardiopulmonary bypass (table 11.1). At the end of the operation systolic and diastolic arterial pressures were slightly lower in the computer-controlled group (table 11.1). Results of the regression analysis are given in figure 11.1. The standard deviation of the 5 -second MAP samples was smaller for the computer- than for the manually controlled group; $7.5 \pm 2.2$ versus $8.9 \pm 2.3 \mathrm{mmHg}(p<0.0001)$. There were fewer hypertensive episodes for the computer- than for the manually controlled group; $9.4 \pm 5.7$ versus $13.1 \pm 6.0 \%$ of the time $(p<0.0001)$. There were fewer hypotensive episodes in MAP for the computer- than for the manually controlled group; $8.0 \pm 5.9$ versus $11.8 \pm 7.4 \%$ of the time $(p<0.0001)$.

\subsection{Discussion}

Computer control of systemic hypertension during cardiac surgery improved haemodynamic stability and reduced the duration of hypertensive and hypotensive episodes. We defined haemodynamic stability as the variation around the average MAP before and after cardiopulmonary bypass. This indirectly assumed that the observed average MAP before and after cardiopulmonary bypass was equal to the desired level of MAP for a given 
patient. This is a reasonable assumption considering the average duration of the operation before and after cardiopulmonary bypass (62 and $49 \mathrm{~min}$ respectively). Furthermore, the analysis of the variations around the average MAP rather than a predefined target allowed for an equal measure of stability in both patient groups.

Both patients group were comparable in patient age and gender and type of surgery. There were no significant differences between patient groups in the average MAP levels before and after cardiopulmonary bypass and in the haemodynamic baseline measurements taken at the start of the operation (table 11.1). Haemodynamic data of our patient group were comparable with haemodynamic data previously published in a multi-centre study (Jain et al. 1997). In the computer-controlled group SNP was used more frequently and higher SNP infusion rates were needed than in the manually controlled group. These underlying differences between patient groups were corrected for by considering the average drug infusion rates as factors in the linear regression models, though the regression models might still have been biased by unmodelled higher-order effects.

For the postoperative cardiac surgical patient, Chitwood et al. (1992) showed that more stable haemodynamics, obtained by computer control, significantly improve patient outcome by reducing blood loss, transfusion requirements and duration of stay in the intensive care unit. The effects of intraoperative haemodynamic stability on patient outcome are less well defined, which might partly be explained by the fact that only recently have objective methods been proposed for qualifying haemodynamic stability during surgery (Reich et al. 1997). Systemic hypertension increases postoperative blood loss (Simpson 1992) and systemic hypertension resulting from an increased systemic resistance increases cardiac afterload and thus left ventricular stroke work. It seems reasonable to assume that hypertensive episodes of short duration will at least increase intraoperative blood loss. Systemic hypotension impairs coronary perfusion, though no significant relation could be found between the number of short hypotensive episodes and the incidence of myocardial ischaemia in patient undergoing cardiac surgery (Jain et al. 1997).

We conclude that computer control of systemic hypertension significantly improves haemodynamic stability. True improvement in patient outcome can however only be expressed in variables that express clinical outcome, such as amount of blood lost, incidence of myocardial ischaemia, duration of hospital stay and improvement of cardiac function. A large patient population receiving different anaesthetic managements and preferably in a multi-centre trial setting is needed to demonstrate a real improvement in 
clinical outcome. The results of the present study motivate the initiation of a multi-centre trial comparing computer versus manual control of systemic hypertension during cardiac surgery, as was conducted by Chitwood et al. (1992) for the postoperative patient. 
Chapter 12

General discussion 
The number of patients with end-stage cardiac failure scheduled for cardiac surgery is increasing. Management of these critically ill patients during and shortly after surgery requires continuous monitoring and control of cardiac pump function. Inotropic and vasoactive drugs are used to optimize cardiac contractility, preload and afterload. Closed-loop control has been proposed to relieve the anaesthesiologist of the time-consuming task of adjusting drug infusion rates. In the present study we have investigated whether closed-loop control can be used to stabilize systemic arterial and pulmonary artery blood pressures simultaneously. We have made no attempt to control cardiac output, because we consider the techniques currently available for monitoring cardiac output in haemodynamically unstable patients continuously are as yet insufficiently reliable. We evaluated closed-loop blood-pressure controllers clinically during cardiac surgery because a faster speed of control is needed here and the incidence and the influence of unmodelled disturbances is greater than in the intensive care unit.

A closed-loop blood-pressure control system needs to test the validity of the measured pressure signal before calculating a new drug infusion rate. For systemic arterial pressure signals at a regular rate, the beat-to-beat fluctuations in a set of characteristic features, including systolic and diastolic pressure and timing values, are analysed to reject those pressure beats that deviate substantially from previously recorded pressure beats (Blom 1990). The pulmonary artery pressure signal was validated in a similar manner by first filtering out the beat-to-beat fluctuations caused by the ventilation (chapter 3). As a first step towards a signal validation algorithm for pressure signals at an irregular rate, a new method of detecting the duration of left ventricular ejection from the radial artery pressure waveform was introduced (chapter 4). This method employs a three-element windkessel model to calculate the shape of the arterial flow waveform from the assessed radial pressure waveform. End-ejection is detected at the first negative dip in the flow waveform. The detection method proved to be robust and relatively insensitive to algorithm design parameters. Next, we demonstrated that detection of pressure beats from pressure signals at an irregular rate is facilitated by simultaneously analysing both systemic arterial and pulmonary artery pressures (chapter 5). The durations of left and right ventricular ejection as derived from the systemic arterial and pulmonary artery pressure signals provided valuable validation criteria for pressure beats at an irregular rate. However, further evaluation of the technique for validation of pressure beats at an irregular rate is needed.

Closed-loop blood-pressure controllers need to adapt themselves to the 
characteristics of the patient under control. With adaptive control it is assumed that the parameters of the patient model describing the response to changes in drug infusion rate can be identified during a control session within a relatively short time-span. Controllability and identifiability, however, are contradictory and a controller with a clinically acceptable control performance may not hold sufficient information for reliable model identification. Many adaptive blood-pressure control concepts, based on established techniques from industrial engineering, have not yet demonstrated their adaptability to clinical applications. This suggests that in a clinical setting the prevalent background noise and unmodelled disturbances may prevent accurate identification of the patient model. This complication was in fact confirmed by our failure to extract sufficient information from closed-loop controlled blood-pressure data (chapter 6). The amount of information extracted during a control session should therefore be reduced. In our control design it was limited to the classification of the patient's sensitivity in one of five sensitivity categories. This provided sufficient information to control systemic arterial (chapters $\tau$ and 8 ) and pulmonary artery blood pressures (chapter 9) adequately during cardiac surgery in all patients. A nearly similar control design was successfully used for a variety of vasoactive drugs, including nitroglycerin, sodium nitroprusside, noradrenaline and dobutamine. This indicates that the infusion of other short-acting vasoactive drugs can also be automated using a similar control design, provided that their response can be approximated by a first-order model.

For the simultaneous stabilization of systemic arterial and pulmonary artery pressures, we avoided the problem of identifying drug cross-effects by only allowing a single drug infusion rate to be changed at a time. A set of priority rules, based on protocols from routine clinical practice, automatically activated online only the optimum single-drug controller for the given haemodynamic state. This strategy was based on our observation that an anaesthesiologist will also try to avoid simultaneous changes in drug infusion rates by controlling the most critical haemodynamic variables first. This multiple-drug control strategy proved feasible in a clinical setting, thereby fulfilling the main goal of the dissertation (chapter 1). As soon as a reliable continuous cardiac output measurement technique is available, a single-drug controller for the control of cardiac output may be developed and integrated into the multiple-drug infusion system by adding priority rules.

Automated infusion of vasodilator drugs in postoperative cardiac surgical patients in the intensive care unit reduces nursing time spent adjusting 
drug infusion rates (Mitchell 1982; Cosgrove III et al. 1989) and improves patient outcome (Chitwood et al. 1992). In chapter 11 it was demonstrated that closed-loop control of systemic arterial pressure during cardiac surgery results in more stable haemodynamic profiles, suggesting a positive effect on patient outcome. Automated control of blood pressure is still not readily accepted in routine clinical practice, however, which might partly be explained by the few studies demonstrating clinical feasibility in large patient-groups and by the even fewer studies reporting effects on patient outcome. Furthermore, the average clinician is not familiar with the concept of computer-control and might fear that he will become redundant as soon as some of his tasks are taken over by a computer-controlled device. The introduction of new equipment therefore calls for special attention. The clinician should be well-informed about the possibilities, but also about the limitations, of the device. This requires from the clinician sufficient additional technical insight into and from the engineer some familiarity with normal working procedures in clinical practice.

Safety aspects play an important part in the acceptance of computerized systems (Bazaral and Ciarkowski 1995). The clinician should feel confident that the closed-loop system can be operated safely under all circumstances. We used a supervisory safety shell to minimize the chance of undesired responses. The supervisory safety shell used expert knowledge to define the closed-loop system's response during sudden large changes in blood pressure, infusion pump problems and invalid pressure measurements. The expertise implemented in the safety shell proved adequate during all clinical trials.

In the present study we demonstrated that closed-loop control of systemic arterial and pulmonary artery blood pressures is feasible during cardiac surgery and displays an improvement in haemodynamic stability when compared with manual control. During the clinical evaluations we used a single anaesthetic protocol; future research should include additional anaesthetic protocols. But most importantly, the impact of closed-loop control during cardiac surgery should further be established, preferably in a multicentre setting as conducted by Chitwood et al. (1992) for the control of postoperative hypertension. 
Appendix

Implementation of expert knowledge 
All blood-pressure controllers, described in this dissertation, were programmed in Pascal (Turbo Pascal v7.0, Borland). Pascal features modular programming by allowing the creation of a sub-program, or unit, that can be included in the main program without affecting the actual implementation of the unit (Borland international 1992). Units are helpful in dividing large programming code into logically related modules. In our case, the main program contained the actual control algorithm and separate units defined procedures to handle communication with infusion pumps, to control the analog-to-digital convertor, to test the validity of the pressure signal and to handle user input and output. The control algorithm was based mainly on knowledge from human experts. The expert knowledge analysed all available information and defined actions for each new time context, allowing the blood-pressure controller to mimic some sort of human reasoning.

Implementing expert knowledge in a formal programming language as Pascal is cumbersome and the implementation itself is difficult to interpret. For these reasons the blood-pressure controller originally described by Blom (1991) used SIMPLEXYS, a general purpose toolbox to design real-time expert system applications (Blom 1990). SIMPLEXYS was originally designed also for monitoring applications and could be further simplified for our control application. We therefore had to create a new Pascalenvironment that facilitated implementation of human reasoning. Unlike SIMPLEXYS (Blom 1991) we decided to use two-valued logic (true or false) instead of three-valued logic (true, unknown or false), because we felt that reasoning with uncertainty is not appropriate in a critical environment where the worst-case scenario should always be assumed. The standard boolean evaluation from Pascal (Borland international 1992) was used to provide (true or false) conclusions derived from basic information regarding the patient or the control system. Object-oriented programming (Borland international 1992) was used to define a rule and a state type, enabling the reasoning with boolean evaluations in a dynamic time context.

\section{Rules}

A rule has a boolean variable to store the conclusion of an evaluation and an integer variable to time how long the evaluation has been true or false. A rule provides a (true or false) conclusion at every time step. A rule is thus evaluated every 5 seconds. The operator Test is used to evaluate a rule. For example,

MapHypertensive.Test(Map> MapMax) 
evaluates to true when mean arterial pressure is hypertensive and to false when it is not hypertensive. MapMax is a (fixed or variable) limit above which the mean arterial pressure is considered to be hypertensive. A rule evaluation can use the values of any valid boolean evaluation (including the conclusions of other rules and states). Each rule has a unique conclusion. The operator Bool returns the (true or false) conclusion of the rule at that moment. For instance, the function call

IneffectiveControl.Bool

returns the conclusion true if control is ineffective and the conclusion false if control is not ineffective. The operator True returns the time in seconds that a conclusion has been true. For example the boolean evaluation,

\section{MapInvalid.True $>60$}

returns the conclusion true when the pressure measurement has been invalid for more than 60 seconds. Similarly, the operator False returns the time in seconds that a conclusion has been false. When a conclusion returns the value true (false) the operator False (True) will evaluate to 0.

The following (simplified) example, illustrates part of the control gain adaptation mechanism implemented using the rule type.

GainUpRequest.Test(

(no_progress_of_pressure) and

(GainConstant.True > 240) and

(FlowMin.False $>60$ ) and

(FlowMax.False $>60$ ) and

(NoTransient.True $>180$ ) and

(not IneffectiveControl.Bool) and

(AutoMode.True>200)
$\{$ Result of function call $\}$ \{ Is gain constant for some time \{Flow rates are not limited

\{No sudden changes in MAP $\}$ \{Drug is effective in patient \{ Is control in automatic mode $\}$ ) ;

Conclusions of rules that are used in the evaluation of the current rule need to be known, forcing the user to start the inference process with primitives at the textual start of the rule base and final evaluations at the textual end of the rule base. All rules are evaluated at each time step to ensure that time-keeping of the rules is always updated, unlike SIMPLEXYS, were rules may also be evaluated conditionally.

\section{States}

A state stores context information and numerical information of how long (i.e. how many seconds) that state held already had the conclusion true or false. State transitions are performed by procedures of the form

On_From_To(Trigger, State1, State2) 
which causes State1 to become false (i.e. inactive) and State2 to become true (i.e. active), if the boolean evaluation Trigger is true and State1 and State 2 evaluate to true and false, respectively. The function

From_To(State1, State2)

evaluates to true if the state transition occurred and false if it did not occur. The default value of a state variable at startup is always false, but can be set to true using the operator InitState. The operation of the operators Bool, True and False is the same for states as for rules. Unlike with rules, however, the conclusion of an evaluation is only available at the next timestep, since the actual state transitions are performed at the end of the current time context.

The state type allows the implementation of hysteresis because different conditions can be used to trigger a state to true (active) and false (inactive). In the example

On_From_To(Map-Target< -4, MapAbove, MapUnder);

On_From_To(Map-Target $>$ 4, MapUnder, MapAbove);

it is tested whether mean arterial pressure crossed the target pressure, taking into account a noise level of $4 \mathrm{mmHg}$ above and below the target pressure. This construction was used to count the number of oscillations of mean arterial pressure around the target pressure. The state type can also be used to solve time-sequence problems like

On_From_To(State1.True >200, State1, State2);

Note that the above type of problem requires memory and cannot be solved using rules.

\section{Actions based on evaluation results}

The rule and the state types are used to implement the evaluation process and the standard if-then Pascal-statement is used to define the actions to be taken based on the results of the evaluation process. For example,

if MapInvalid.True> 60 then 'switch to manual mode' will return control to manual mode when the pressure measurement has been invalid for more than 60 seconds. 


\section{Bibliography}

Arnsparger, J. M., B. C. McInnis, J. R. Glover, and N. A. Normann (1983). Adaptive control of blood pressure. IEEE Trans Biomed Eng 30(3), 168-176.

Åström, K. J. and T. Hågglund (1995). PID controllers: Theory, design and tuning. Technical report, Instrument society of America, The international society for measurement and control, Research Triangle Park, North Carolina.

Aström, K. J. and B. Wittenmark (1995). Adaptive control (second ed.). Amsterdam: Addison-Wesley.

Bazaral, M. G. and A. Ciarkowski (1995). Food and drug administration regulations and computer-controlled infusion pumps. International Anesthesiology Clinics 33(3), 45-63.

Beatty, P. C. W. (1993). Software safety considerations in the use of closed-loop, patient-connected control systems. $B r J$ Anaesth 71, 461.

Bednarski, P., F. Siclari, A. Voigt, S. Demertzis, and G. Lau (1990). Use of a computerized closed-loop sodium nitroprusside titration system for antihypertensive treatment after open heart surgery. Crit Care Med 18(10), 1061-1065.

Beppu, T., Y. Imai, and Y. Fukui (1995). A computerized control system for cardiopulmonary bypass. J Thorac Cardiovasc Surg 109(3), 428438.

Blom, J. A. (1990). The SIMPLEXYS experiment. Real time expert systems in patient monitoring. Ph. D. thesis, Eindhoven University of Technology.

Blom, J. A. (1991). Expert control of the arterial blood pressure during surgery. Int $J$ Clin Monit Comput 8, 25-34.

Borland international (1992). Turbo pascal language guide. Scotts valley, CA 95067-0001: Borland international. 
Burkhoff, D., J. Alexander, and J. Schipke (1988). Assessment of windkessel as a model of aortic impedance. Am. J Physiol 255, H742-H753.

Chaudhrim, S., J. R. Colvin, J. G. Todd, and G. N. C. Kenny (1992). Evaluation of closed loop control of arterial pressure during hypotensive anaesthesia for local resection of intraocular melanoma. Brit $J$ Anaesth 69, 607-610.

Chen, C., W. Lin, T. Kuo, and C. Wang (1997). Adaptive control of arterial blood pressure with a learning controller based on multilayer networks. IEEE Trans Biomed Eng 44, 601-609.

Chiariello, M., H. K. Gold, R. C. Leinback, M. A. Davis, and P. R. Maroko (1976). Comparison between the effects of nitroprusside and nitroglycerin on ischemic injury during acute myocardial infarction. Circulation 54, 766-773.

Chitwood, W. R., D. M. Cosgrove III, R. M. Lust, and the Titrator Multicenter Study Group (1992). Multicenter trial of automated nitroprusside infusion for postoperative hypertension. Ann. Thorac Surg 54, $517-522$.

Colvin, J. R. and G. N. C. Kenny (1989). Automatic control of arterial pressure after cardiac surgery, evaluation of a microcomputer-based control system using glyceryl trinitrate and sodium nitroprusside. Anaesthesin 44, 37-41.

Cosgrove III, D. M., J. H. Petre, J. L. Waller, J. V. Roth, C. Shepherd, and L. H. Cohn (1989). Automated control of postoperative hypertension: A prospective, randomized multicenter study. Ann Thorac Surg 47, 678-683.

Csongrady, A. and Ponz-Gonzalez-L (1980). Hypotensive anaesthesia in the oto-rhino-laryngological surgery using nitroglycerine. Anaesthesist 29, 261-265.

Dahlgren, G., F. Veintemilla, G. Settergren, and J. Liska (1991). Left ventricular end-systolic pressure estimated from measurements in a peripheral artery. J Cardiothorac Vasc Anesth 5, 551-553.

de Asla, R. A., A. M. Benis, R. A. Jurado, and R. S. Litwak (1985). Management of postcardiotomy hypertension by microcomputercontrolled administration of sodium nitroprusside. J Thorac Cardiovasc Surg 89(1), 115-120.

Ellis, D. M. (1985). Interpretation of beat-to-beat blood pressure values in the presence of ventilatory changes. $J$ Clin. Monit $1(1), 65-70$. 
Endrich, B., N. Franke, K. Peter, and K. Messmer (1987). Induced hypotension: Action of sodium nitroprusside and nitroglycerin on the microcirculation. Anesthesiology 66, 605-613.

Fahmy, N. R. (1978). Nitroglycerin as a hypotensive drug during general anesthesia. Anesthesiology 49, 17-20.

Foëx, P. (1980). The mechanical effects of raised airway pressure. In C. Prys-Roberts (Ed.), The circulation in anaesthesia, Chapter 13, pp. 281-294. Oxford: Blackwell Scientific Publications.

Friederich, J. A. and J. F. Butterworth (1995). Sodium nitroprusside: Twenty years and counting. Anesth Analg 81, 152-162.

Gessing, R. and M. Blachuia (1996). Set point and identifiability in the closed loop with a minimum-variance controller. Control Eng Practice $4,665-670$.

Gingrich, K. J. and R. J. Roy (1991). Modeling the hemodynamic response to dopamine in acute heart failure. IEEE Trans Biomed Eng 38(3), 267-272.

Gratz, I., J. Kraidin, A. G. Jacobi, N. G. deCastro, P. Spagna, and G. E. Larijani (1992). Continuous noninvasive cardiac output as estimated from the pulse contour curve. $J$ Clin Monit 8, 20-27.

Guggiari, M., F. Dagreou, A. Lienhart, S. Gallais, P. Mottet, J. Philippon, and P. Viars (1985). Use of nitroglycerin to produce controlled decreases in mean arterial pressure to less than $50 \mathrm{~mm}$ hg. $\mathrm{Br} J$ Anaesth 57, 142-147.

Guyton, A. C., T. G. Coleman, A. W. Cowley, J. Liard, R. A. Norman, and R. D. Manning, Jr. (1972). Systems analysis of arterial pressure regulation and hypertension. Ann of Biomed Eng 1, 254-281.

Hammond, J. J., W. M. Kirkendall, and R. V. Calfee (1979). Hypertensive crisis managed by computer controlled infusion of sodium nitroprusside: A model for the closed loop administration of short acting vasoactive agents. Comput Biomed Res 12, 97-108.

He, W. G., H. Kaufman, and R. Roy (1986). Multiple model adaptive control procedure for blood pressure control. IEEE Trans Biomed Eng 33, 10-19.

Held, C. M. and R. J. Roy (1995). Multiple drug hemodynamic control by means of a supervisory-fuzzy rule-based adaptive control system: validation on a model. IEEE Trans Biomed Eng 42(4), 371-384. 
Hempelmann, G., S. Piepenbrock, W. Seitz, and G. Karliczek (1977). Changes in hemodynamic parameters, inotropic state, and myocardial oxygen consumption owing to intravenous application of nitroglycerin. J Thorac Cardiovasc Surg 73, 836-847.

Hewlett Packard (1989). An algorithm for the reduction of the respiration artifact in pulmonary artery pressure measurements. Hewlett Packard. Application note 5953-7352.

Hoffman, B. B. and R. J. Lefkowitz (1996). Catecholamines, sympathomimetic drugs, and adrenergic receptor antagonists. In J. G. Hardman, A. Goodman Gilman, and L. E. Limbird (Eds.), Goodman \& Gilmanis The Pharmacological Basis of Therapeutics (9 ed.)., Chapter 10, pp. 199-248. New York: McGraw-Hill.

Hsia, T. C. (19T7). System identification. Lexington books.

Hunt, K. J., D. Sbarbaro, R. Żbikowski, and P. J. Gawthrop (1992). Neural networks for control systems - a survey. Automatica 28, 10831112.

Incalzi, R. A., A. Gemma, A. Cocchi, and P. Carbonin (1989). Baroreceptor reset with nitroprusside and drug-resistant hypertension. The Lancet 2, 504 .

Isaka, S. and A. V. Sebald (1993). Control strategies for arterial blood pressure regulation. IEEE Trans Biomed Eng 40(4), 353--363.

Jain, U., C. J. A. Laflamme, A. Aggarwal, J. G. Ramsay, M. E. Comunale, S. Ghoshal, L. Ngo, K. Ziola, M. Hollenberg, and D. T. Mangano (1997). Electrocardiographic and hemodynamic changes and their association with myocardial infarction during coronary artery bypass surgery. Anesthesiology 86, 576-591.

Jansen, J. R. C., J. J. Schreuder, J. J. Settels, J. J. Kloek, and A. Versprille (1990a). An adequate strategy for the thermodilution technique in patients during mechanical ventilation. Intensive Care Med 16, 422-425.

Jansen, J. R. C., K. H. Wesseling, J. J. Settels, and J. J. Schreuder (1990b). Continuous cardiac output monitoring by pulse contour during cardiac surgery. Eur Heart $J 11$ Suppl I, 26-32.

Jastremski, M., C. Jastremski, M. Shepherd, V. Friedman, D. Porembka, R. Smith, E. Gonzales, D. Swedlow, H. Belzberg, R. Crass, T. Jannett, E. Richards III, D. Thijs, and D. Woods (1995). A model for 
technology assessment as applied to closed loop infusion systems. Crit Care Med 23, 1745-1755.

Jones, C. J. H. and M. Sugawara (1993). Wavefronts in the aorta implications for the mechanism of left ventricular ejection and aortic valve closure. Cardiovasc Res 2\%. 1902-1905.

Iiaplan, J. A. and A. V. Guffin (1993). Treatment of perioperative left. ventricular failure. In J. A. Kaplan (Ed.). Cardiac Anesthesia (third ed.)., Chapter 32, pp. 1058-1094. Philadelphia: W.B. Saunders.

Tiaplan. J. A. and E. L. Jones (1979). Vasodilator therapy during coronary artery surgery. comparison of nitroglycerin and nitroprusside. $J$ Thorac Cardiovasc Surg 77, 301-309.

Kates. R. E. and C. V. Leier (1978). Dobutamine pharmacokinetics in severe heart failure. Clin. Pharmacol Ther 24(5). 537-541.

Katona, P. G. (1982). Automated control of physiological variables and clinical therapy. CRC Crit Rev Biomed Eng 8. 281-310.

Kininias. P., H. A. Fozzard, and M. J. Norusis (1981). A real-time pressure algorithm. Comput Biol Med 11, 211-220.

Kuo, T. B. J. and S. H. H. Chan (1993). Continuous, on-line. real-time spectral analysis of systemic arterial pressure signals. Am.J Physiol 264 (Heart Circ. Physiol. 33), H2208-H2213.

Kiwok. K. E., S. L. Shah, A. S. Clanachan, and B. A. Finegan (1995). Evaluation of long-range adaptive predictive controller for computerized drug delivery systems. IEEE Trans Biomed Eng 42(1). 79-86.

Langewouters, G. J., K. H. Wesseling, and W. J. A. Goedhard (1984). The static elastic properties of 45 human thoracic and 20 abdominal aortas in vitro and the parameters of a new model. $J$ Biomech $1 \%$ $425-435$.

Laskey, W., H. G. Parker, V. A. Ferrari. W. G. Kussmaul, and A. Noordergraaf (1990). Estimation of total systemic arterial compliance in humans. J Appl Physiol 69, 112-119.

Latson, T. W. W. C. Hunter, N. Tiatoh, and K. Sagawa (1988). Effect of nitroglycerin on aortic impedance, diameter, and pulse-wave velocity. Circ Res 62, 884-890.

Lewis. R. P., S. E. Rittgers, W. F. Forester, and H. A. Boudoulas (19-i ). Critical review of the systolic time intervals. Circulation 56.146-158. 
Li. J. K. J., T. Cui, and G. M. Drzewiecki (1990). A nonlinear model of the arterial system incorporating a pressure-dependent compliance. IEEE Trans Biomed Eng 37, 673-678.

Linkens, D. A. and S. S. Hacisalihzade (1990). Computer control systems and pharmacological drug administration: a survey. J Med Eng Tech 14(2), 41-54.

Liu, Z., K. P. Brin, and F. C. P. Yin (1986). Estimation of total arterial compliance: an improved method and evaluation of current methods. Am. J Physiol 251, H588-H600.

Ljung, L. (1987). System identification - Theory for the user. PrenticeHall.

Ljung, L. and T. Söderström (1983). Theory and practice of recursive identification. The MIT Press series in signal processing, optimization, and control. The Massachusetts Institute of Technology: MIT Press.

Maitelli, A. L. and T. Yoneyama (1997). Suboptimal dual adaptive control for blood pressure management. IEEE Trans Biomed Eng 44, 486-492.

Mangano, D. T., D. Siliciano, M. Hollenberg, J. M. Leung, W. S. Browner, P. Goehner, S. Merrick, E. Verrier, and the study of Perioperative Ischemia (SPI) Research Group (1992) Postoperative myocardial ischemia (1992). Therapeutic trials using intensive analgesia following surgery. Anesthesiology 76, 342-353.

Martin, J. F., A. M. Schneider, M. L. Quinn, and N. T. Smith (1992a). Improved safety and efficacy in adaptive control of arterial blood pressure through the use of a supervisor. IEEE Trans Biomed Eng 39(4), 381-388.

Martin, J. F., A. M. Schneider, and N. T. Smith (1987). Multiple-model adaptive control of blood pressure using sodium nitroprusside. IEEE Trans Biomed Eng 34(8), 603-611.

Martin, J. F., N. T. Smith, M. L. Quinn, and A. M. Schneider (1992b). Supervisory adaptive control of arterial pressure during cardiac surgery. IEEE Trans Biomed Eng 39(4), 389-393.

Marwick, T. H. (1995). Arbutamine stress testing with closed-loop drug delivery. JACC 26(5), 1176-1179. Editorial comment.

McInnis, B. C. and L. Z. Deng (1985). Automatic control of blood pressures with multiple drug inputs. Ann Biomed Eng 13, 217-225. 
Meijers, R. H. A. M., D. Schmartz, F. R. L. Cantraine, L. Barvais, A. A. d'Hollander, and J. A. Blom (1997). Clinical evaluation of an automatic blood pressure controller during cardiac surgery. $J$ Clin Monit 13, 261-268.

Meline, L. J., D. R. Westenskow, N. L. Pace, and M. N. Bodily (1985). Computer-controlled regulation of sodium nitroprusside infusion. Anesth Analg 64, 38-42.

Millard, R. K., P. Hutton, E. Pereira, and C. Prys-Roberts (1987). On using a self-tuning controller for blood pressure regulation during surgery in man. Comput Biol Med 17(1), 1-18.

Miller, R. R., N. A. Awan, J. A. Joye, K. S. Maxwell, A. N. DeMaria, E. A. Amsterdam, and D. T. Mason (1977). Combined dopamine and nitroprusside therapy in congestive heart failure. Circulation 55(6), 881-884.

Mitchell, R. R. (1982). The need for closed-loop theraphy. Crit Care Med 10(12), 831-834.

Nichols, W. W., M. F. O'Rourke, and R. McDonald (1990). McDonald's blood flow in arteries. London: Edward Arnold.

Ocasio, W. C., D. R. Rigney, K. P. Clark, and R. G. Mark (1993). bpshape_wk4: a computer program that implements a physiological model for analyzing the shape of blood pressure waveforms. Comput Method Prog Biomed 39, 169-194.

Odaka, T., H. Agata, H. Furutani, and H. Ogura (1994). A general purpose neural network simulator system for medical data processing. $J$ Med Sys 18(6), 305-314.

O'Hara, D. A., D. K. Bogen, and A. Noordergraaf (1992). The use of computers for controlling the delivery of anesthesia. Anesthesiology 77(3), $563-581$.

Oppenheim, A. V., A. S. Willsky, and I. T. Young (1983). Signals and systems, Chapter 10, pp. 665-667. Prentice-Hall International, Inc.

Oppenheim, M. I. and D. F. Sittig (1995). An innovative dicrotic notch detection algorithm which combines rule-based logic with digitial signal processing techniques. Comput Biomed Res 28, 154-170.

Packer, J. S., D. G. Mason, J. F. Cade, and S. M. McKïinley (1987). An adaptive controller for closed-loop management of blood pressure in seriously ill patients. IEEE Trans Biomed Eng 34(8), 612-616. 
Papoulis, A. (1991). Probability, random variables, and stochastic processes. Chapter 16. pp. 603-612. Singapore: McGraw-Hill.

Parkin, G., C. Wright, R. Bellomo, and N. Boyce (1994). Use of mean systemic filling pressure analogue during the closed-loop control of fluid replacement in continuous hemodiafiltration. J Crit Care 9(2), 124-133.

Pichlmaier. A. M., D. Braile, E. Ebner, O. T. Greco, H. Hutten, G. H. von Iinorre, W. Niederlag, W. Rentsch, H. Volkmann, D. Weber, E. Wunderlich, and M. Schaldach (1992). Autonomic nervous system controlled closed loop cardiac pacing. PACE 15, 1787-1791.

Pike, T. and A. Mustard (1992). Automated recognition of corrupted arterial waveforms using neural network techniques. Comput Biol Med 22(3), 173-179.

Prentza, A. and K. H. Wesseling (1995). Catheter-manometer system damped blood pressures detected by neural nets. Med Biol Eng Comput 33, 589-595.

Prys-Roberts, C. and R. K. Millard (1990). Self-tuning adaptive control of induced hypotension in humans: A comparison of isoflurane and sodium nitroprusside. J Clin Monit 6(3), 236-240.

Reich, D. L., T. I. Osinkski, C. Bodian, M. Krol, K. Sarier, R. Roth, and S. N. Konstadt (1997). An algorithm for assessing intraoperative mean arterial pressure lability. Anesthesiology 87, 156-161.

Reid, J. A. and G. N. C. Kenny (1987). Evaluation of closed-loop control of arterial pressure after cardiopulmonary bypass. $\mathrm{Br} J$ Ancuesth 59 , $247-255$.

Rosenfeldt, F. L., V. Chang, M. Grigg, S. Parker, R. Cearns, M. Rabinov, and W. G. Xu (1986). A closed loop microprocessor controller for treatment of hypertension after cardiac surgery. Ancuesth Intens Care 14(2), 158-162.

Ruiz. R., D. Borches, A. González, and J. Corral (1993). A new sodiumnitroprusside-infusion controller for the regulation of arterial blood pressure. Biomed Inst Tech 27, 244-251.

Sakamoto, T., H. Arai, T. Maruyama, and A. Suzuki (1995). New algorithm of intra aortic balloon pumping in patients with atrial fibrillation. ASAIO J 41, 79-83.

Schwid, H. A., L. A. Taylor, and N. T. Smith (1987). Computer model 
analysis of the radial artery pressure waveform. J Clin Monit 3, 220228.

Sheppard, L. C. (1980). Computer control of the infusion of vasoactive drugs. Ann Biomed Eng 8, 431-444.

Sheppard, L. C., N. T. Kouchoukos, M. A. Kurtts, and J. W. Kïklin (1968). Automated treatment of critically ill patients following operation. Annals of Surgery 168, 596-604.

Simpson, P. (1992). Perioperative blood loss and its reduction: the role of the anaesthetist. Brit $J$ Anaesth 69, 498-507.

Smith, N. T. and H. O. Schwede (1972). The response of arterial pressure to halothane: a systems analysis. Med \& Biol Engng 10, 207-221.

Starmer, C. F., P. A. McHale, and J. C. Greenfield (1973). Processing of arterial pressure wave with a digital computer. Comput Biomed Res 6, 90-96.

Stern, K. S., H. J. Chizeck, B. K. Walker, P. S. Krishnaprasad, P. J. Dauchot, and P. G. Katona (1985). The self-tuning controller: comparison with human performance in the control of arterial pressure. Ann Biomed Eng 13, 341-357.

Stinson, L. W., M. J. Murray, K. A. Jones, S. J. Assef, M. J. Burke, T. L. Behrens, and R. L. Lennon (1994). A computer-controlled, closed-loop infusion system for infusing muscle relaxants: Its use during motor-evoked potential monitoring. J Cardiothorac Vasc Anesth 8(1), 40-44.

Sunagawa, K. and K. Sagawa (1982). Models of ventricular contraction based on time-varying elastance. Crit Rev Biomed Eng 7, 193-228.

TenVoorde, B. J., T. J. C. Faes, and O. Rompelman (1994a). Spectra of data sampled at frequency-modulated rates in application to cardiovascular signals: Part 1 analytical derivation of the spectra. Med Biol Eng Comput 32, 63-70.

Ten Voorde, B. J., T. J. C. Faes, and O. Rompelman (1994b). Spectra of data sampled at frequency-modulated rates in application to cardiovascular signals: Part 2 evaluation of fourier transform algorithms. Med Biol Eng Comput 32, 71-76.

Todd, M. M., P. J. Morris, J. Moss, and D. M. Philbin (1982). Hemodynamic consequences of abrupt withdrawal of nitroprusside or nitroglycerin following induced hypotension. Anesth Analg 61 (3), 261-266. 
van der Hoeven, G. M. A., P. J. A. Clerens, J. J. H. Donders, J. E. W. Beneken, and J. T. C. Vonk (1977). A study of systolic time intervals cluring uninterrupted exercise. Br Heart $J$ 39, 242-254.

Voss, G. I., P. G. Katona, and H. J. Chizeck (1987). Adaptive multivariable drug delivery: Control of arterial pressure and cardiac output in anesthetized dogs. IEEE Trans Biomed Eng 34(8), 617-623.

Wesseling, K. H., J. R. C. Jansen, J. J. Settels, and J. J. Schreuder (1993). Computation of aortic flow from pressure in humans using a nonlinear, three-element model. J Appl Physiol 74, 2566-2573.

Wood, M., S. Hyman, and A. J. J. Wood (1987). A clinical study of sensitivity to sodium nitroprusside during controlled hypotensive anesthesia in young and elderly patients. Anesth Analg 66, 132-136.

Woolfson, M. S. (1991). Study of cardiac arrhythmia using the kalman filter. Med Biol Eng Comput 29, 398-405.

Ying, H., M. McEachern, D. W. Eddleman, and L. C. Sheppard (1992). Fuzzy control of mean arterial pressure in postsurgical patients with sodium nitroprusside infusion. IEEE Trans Biomed Eng 39(10), 1060-1070.

Yu, C., R. J. Roy, H. Kaufman, and B. W. Bequette (1992). Multiplemodel adaptive predictive control of mean arterial pressure and cardiac output. IEEE Trans Biomed Eng 39(8), 765-778.

Zadeh, L. A. (1978). Fuzzy sets as a basis for a theory of possibility. Fuzzy sets and Systems 1, 3-28.

Zbinden, A. M., P. Feigenwinter, S. Petersen-Felix, and S. Hacisalihzade (1995). Arterial pressure control with isoflurane using fuzzy logic. $\mathrm{Br}$ $J$ Ancuesth 74, 66-72.

Zelano, J. A. (1986). The computer control of intra-aortic balloon pumping. Ph. D. thesis, Rutgers University.

Zelano, J. A., W. Ko, R. Lazzaro, W. Lazenby, S. Topaz, W. J. Kolff, O. W. Isom, and K. H. Krieger (1992). Evaluation of an extraaortic counterpulsation device in severe cardiac failure. Ann Thorac Surg 53, $30-37$.

Zelano, J. A., J. K. J. Li, and W. Welkowitz (1990). A closed-loop control scheme for intraaortic balloon pumping. IEEE Trans Biomed Eng 37(2), 182-192. 


\section{Summary}

During and shortly after cardiac surgery, cardiac pump function is evaluated from systemic and pulmonary artery blood-pressure measurements and intermittent cardiac output measurement. One of the tasks of the anaesthesiologist is to ensure that all variables remain within an acceptable range by infusing vasoactive and contractility enhancing drugs. Closed-loop control by computer has been proposed to relieve the anaesthesiologist of the timeconsuming task of continuously adjusting drug infusion rates. Closed-loop control involves automatic assessment of mean blood pressure and calculation of the drug infusion rate needed to maintain the pressure around a target level. Drugs are administered to the patient by a computer-controlled infusion pump. Closed-loop control is presently limited to systemic and pulmonary pressures, because the techniques currently available for assessing cardiac output do not enable reliable continuous measurement. The aim of the present study was to develop and clinically evaluate an automated multiple-drug infusion system that simultaneously stabilizes systemic and pulmonary blood pressures.

Blood pressures are routinely measured invasively by means of external pressure transducers and a fluid-filled line connected to a radial artery cannula or a pulmonary artery catheter. Signal validation algorithms are needed to ensure that the closed-loop system does not respond to corrupted pressure measurements. Validation techniques for the systemic pressure analyse the beat-to-beat fluctuations in a set of characteristic features, including systolic and diastolic pressures and timing values, to reject those pressure beats that deviate too strongly from previous pressure beats. Interpretation of pulmonary artery blood pressures is difficult owing to the respiration artefact which modulates both offset and amplitude of the pulmonary artery pressure signal. In chapter 3 an adaptive filtering technique was proposed that corrects features of pulmonary artery pressure beats for the cyclic disturbances that are induced by the ventilation. The pulmonary artery pressure signal could adequately be validated by analysing 
the beat-to-beat fluctuations in the corrected pulmonary features.

Pressure signals at an irregular rate exhibit large fluctuations in shape between beats and cannot be validated using the techniques described above. As a first step towards a signal validation algorithm for pressure signals at an irregular rate we investigated in chapter 4 whether end-ejection can be detected reliably from the radial artery pressure signal. The proposed method detects the dicrotic notch from the shape of the arterial flow waveform which is obtained from simulation results with a three-element windkessel model. The detection algorithm proved robust and relatively insensitive to the clesign parameters of the algorithm.

We demonstrated in chapter 5 that cletection of pressure beats from pressure signals at an irregular rate is facilitated by simultaneously analysing both systemic and pulmonary pressures. Furthermore the durations of left and right ventricular ejection, as clerived from the systemic and pulmonary pressure signals, provided valuable criteria for validating pressure beats at an irregular rate. However, the validation technique for pressure beats at an irregular rate should be further evaluated.

Closed-loop blood-pressure controllers adapt themselves to the characteristics of the patient under consideration. For adaptive control it is assumed that the parameters of the patient model, describing the response to changes in clrug infusion rate, can be estimated during a control session. Controllability and identifiability are however contradictory and in chapter 6 it was clemonstrated that an adaptive blood-pressure controller with a clinically acceptable control performance might not collect sufficient information for reliable model identification. In our clesign, we limited the online model iclentification to the classification of the patient's sensitivity in one of five categories. Rule-based logic was used to classify the patient's sensitivity and to define a supervisory safety shell. This blood-pressure control strategy was successfully used during cardiac surgery to control systemic hypertension using nitroglycerin (chapter $T$ ) and sodium nitroprusside (chapter 8) and to control pulmonary artery hypertension using nitroglycerin (chapter 9 ). The speed of each closed-loop system was adequate. The expert knowledge that was implemented in the supervisory safety shell proved to be correct in all cases, since manually overruling the control system was never cleemed necessary.

Control of a single blood pressure using a single drug does not control overall haemodynamics. Repeated adjustments of both vasoactive and inotropic drug infusion rates are needed to maintain systemic and pulmonary pressures within an acceptable range, especially in the haemodynamically unstable patient (e.g. suffering from heart failure). We developed a control 
system for the simultaneous control of systemic and pulmonary pressures by integrating several single-drug blood-pressure controllers into one multipleinput multiple-output system (chapter 10). A set of priority rules automatically activated only the optimum single-drug controller for the given haemodynamic state. This strategy was based on our observation that an anaesthesiologist also tries to avoid simultaneous changes in drug infusion rates by controlling the most critical haemodynamic variables first. Furthermore we avoided the difficulty of identifying drug cross-effects by only allowing a single drug infusion rate to be changed at a time. The multipledrug infusion system was evaluated during cardiac surgery in thirty patients who required simultaneous treatment with more than one vasoactive or inotropic drug. The activation of the single-drug controllers by the priority rules was both accurate and sufficiently fast. None of the single-drug controllers showed any sign of unstable behaviour.

In chapter 11 it was demonstrated that closed-loop control of systemic pressure results in more stable haemodynamic profiles as compared to manual control. Moreover, there were fewer hypertensive and hypotensive episodes in the computer than in the manually controlled group. True improvement in patient outcome can however only be expressed in variables that express clinical outcome, such as amount of blood loss, incidence of myocardial ischaemia, duration of hospital stay and improvement in cardiac function. Further research, preferably in a multi-centre setting, is needed to study the effects of closed-loop blood-pressure control on patient outcome.

In conclusion, we described a closed-loop system that accurately controls systemic and pulmonary artery blood pressures during cardiac surgery by automating the infusions of vasoactive and contractility enhancing drugs. A set of priority rules analysed the patient's haemodynamic state online and enabled us to control systemic and pulmonary pressures simultaneously. A supervisory safety shell ensured a safe response under all circumstances. Signal validation algorithms ensured that the closed-loop system did not respond to invalid pressure measurements, although the validation of pressure signals at an irregular rate needs to be further improved. Preliminary investigations indicated an improvement in patient outcome when pressures were controlled by computer. 


\section{Dankwoord}

Het in dit proefschrift beschreven onderzoek was natuurlijk nooit tot stand gekomen zonder gedegen begeleiding en steun van verschillende mensen.

In de allereerste plaats wil ik Jan Schreuder bedanken voor zijn prettige manier van begeleiden. Jan heeft me wegwijs gemaakt in de klinische wereld en heeft mij veel geleerd over het opzetten en bedrijven van wetenschappelijk onderzoek. Verder bleef Jan altijd enthousiast voor ons onderzoek en wist hij een werksfeer te creëren waarbinnen ik in alle vrijheid heb kunnen werken.

Hans Blom wil ik bedanken voor zijn creatieve inbreng en steun door de jaren heen. Tijdens zijn wekelijkse bezoeken vormde hij de voor mij noodzakelijk brug met de technische achterban. Ook zou zonder zijn voorbereidend werk het in dit proefschrift beschreven onderzoek nooit tot stand zijn gekomen.

De promotores Arnold Hoeks en Rob Reneman wil ik bedanken voor hun heldere en duidelijke commentaren die er voor gezorgd hebben dat het proefschrift ook voor een breder publiek begrijpbaar is geworden.

De leden van de beoordelingscommissie, prof. dr. ir. A. Hasman, prof. dr. ir. T. G. J. Arts, prof. dr. J. G. Bovill, prof. dr. ir. P. P. J. van den Bosch en Prof. dr. P. W. de Leeuw, wil ik bedanken voor het kritisch doorlezen van het manuscript. In het bijzonder wil ik Prof. dr. ir. A. Hasman bedanken voor het feit dat Hans Blom tijd vrij kon maken van zijn werkzaamheden bij de vakgroep Medische Informatica om mij wekelijks met daad en raad bij te staan.

Jos Jansen (Longziekten, Erasmus universiteit) wil ik bedanken voor zijn steun en voor de inbreng van zijn kennis over de interpretatie van bloeddruksignalen.

Verder ben ik de afdeling cardiopulmonale chirurgie en in het bijzonder Jos Maessen dankbaar voor het vertrouwen dat ze in onze systemen hebben gesteld en voor het feit dat we deze in een klinische omgeving hebben kunnen evalueren. Ook wil ik alle anaesthesieverpleegkundigen bedanken 
voor hun inzet en voor hun begrip als de 'karren van jan' weer binnen kwamen rijden.

Mijn kamergenoten door de jaren heen, Theo, Ruud, Jo en Erik, wil ik bedanken voor hun steun, de gezellige lunches en de onontbeerlijke koffiepauzes. Matthijs, bedankt dat je na het jarenlang aanhoren van het wel en wee van mijn onderzoek mij ook tijdens de verdediging bij wilt staan. Tenslotte wil ik mijn familie bedanken voor de steun die ze me de afgelopen jaren vanuit het thuisfront hebben gegeven. 


\section{Curriculum Vitea}

1968 Geboren op twee september te Geleen.

1981-1987 Atheneum $\beta$ aan het Henric van Veldeke College te Maastricht.

1987-1992 Studie elektrotechniek, regel- en systeemtechniek, aan de Technische Universiteit Eindhoven.

1993-1994 Werkzaam als erkend gewetensbezwaarde militaire dienst voor de afdelingen cardiochirurgie en anesthesiologie van het academisch ziekenhuis Maastricht.

1994-1998 Promotieonderzoek bij de projectgroep pompfunktie monitoring en regulatie, vakgroep anaesthesiologie, cardiovasculair research instituut Maastricht, universiteit Maastricht. 
170 


\section{List of publications}

\section{Articles}

Hoeksel, S. A. A. P. (1995). Computergestuurde toediening van vasoactieve medicamenten gedurende en na open hartoperaties. NeSECC Journal 20(1), 10-12. (in Dutch)

Hoeksel, S. A. A. P., J. A. Blom, J. R. C. Jansen, and J. J. Schreuder (1996). Correction for respiration artifact in pulmonary blood pressure signals of ventilated patients. J Clin Monit 12(5), 397-403.

Hoeksel, S. A. A. P., J. J. Schreuder, J. A. Blom, J. G. Maessen, and O. C. K. M. Penn (1996). Automated infusion of nitroglycerin to control arterial hypertension during cardiac surgery. Intensive Care Med 22(7), 688-693.

Hoeksel, S. A. A. P., J. R. C. Jansen, J. A. Blom, and J. J. Schreuder (1997). Detection of dicrotic notch in arterial pressure signals. $J$ Clin Monit 13(5), 309-316.

Schreuder, J. J., F. H. van der Veen, E. T. van der Velde, F. Delahaye, O. Alfieri, O. Jegaden, R. Lorusso, J. R. C. Jansen, S. A. A. P. Hoeksel, G. finet, M. Volterrani, H. -G. Kaulbach, J. Baan, and H. J. J. Wellens (1997). Left ventricular pressure-volume relationships before and after cardiomyoplasty in patients with heart failure. Circulation 96(9), 2978-2986.

Hoeksel, S. A. A. P., J. A. Blom, J. R. C. Jansen, J. G. Maessen, and J. J. Schreuder (1997). Computer control versus manual control of systemic hypertension during cardiac surgery. Submitted.

Hoeksel, S. A. A. P., J. A. Blom, J. R. C. Jansen, A. P. G. Hoeks, and J. J. Schreuder (1997). The feasibility of adaptive control of blood pressure during cardiac surgery. Submitted. 
Hoeksel, S. A. A. P., J. A. Blom, J. R. C. Jansen, J. G. Maessen, and J. J. Schreuder (1998). Automated infusion vasoactive and inotropic drugs to control arterial and pulmonary pressures during cardiac surgery. Submitted.

Punt, C. D., J. J. Schreuder, J. R. C. Jansen, S. A. A. P. Hoeksel, and A. Versprille (1998). Tracing best PEEP by applying PEEP as a ramp. Submitted.

\section{Abstracts}

Hoeksel, S. A. A. P., J. J. Schreuder, J. G. Maessen, and J. A. Blom (1994). Nitroglycerin automatic control of arterial blood pressure during cardiac surgery. In B. W. Patterson (Ed.), Modeling and Control in Biomedical Systems, pp. 177. International Federation of Automatic Control (IFAC).

Hoeksel, S. A. A. P., J. J. Schreuder, J. A. Blom, J. G. Maessen, and O. C. K. M. Penn (1994). Automated infusion of sodiumnitroprusside during cardiac surgery. In The 14th International Symposium on Computing in Anesthesia and Intensive Care. Dept. of Anesthesiology, Erasmus University Rotterdam.

Hoeksel, S. A. A. P. and J. J. Schreuder (1995). Closed-loop control of left ventricular filling pressure. Pfiügers Archiv: European. Journal of Physiology Suppl. to 430(4), R128.

Hoeksel, S. A. A. P., J. A. Blom, J. G. Maessen, and J. J. Schreuder (1996). Automated infusion of vasodilators to stabilize hemodynamics during cardiac surgery. Cardiovasc Surg 4 (Suppl. no. 1), 170 .

\section{Other}

Hoeksel, S. A. A. P., J. J. Schreuder, and J. R. C. Jansen. Inrichting voor het bepalen van een karakteristiek punt in de hartcyclus. Octrooiaanvraag no. 8529WO (1995). International Application No. PCT/NL96/00490 (1996). 